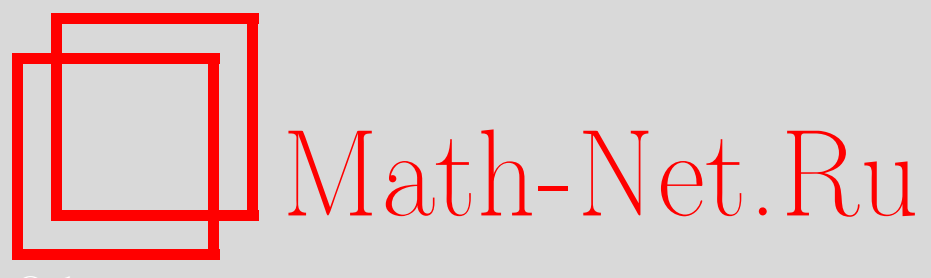

М. Божейко, Е. В. Литвинов, И. В. Родионова, Расширенное энионное фоковское пространство и некоммутативные ортогональные многочлены типа Мейкснера в бесконечномерном случае, УМH, 2015, том 70, выпуск $5,75-120$

DOI: https://doi.org/10.4213/rm9668

Использование Общероссийского математического портала Math-Net.Ru подразумевает, что вы прочитали и согласны с пользовательским соглашением http: //www.mathnet.ru/rus/agreement

Параметры загрузки:

IP : 44.207 .124 .84

26 апреля 2023 г., 13:05:51

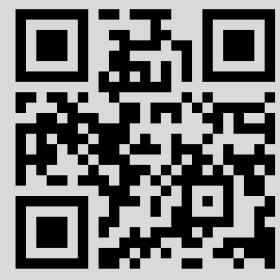




\section{Расширенное энионное фоковское пространство и некоммутативные ортогональные многочлены типа Мейкснера в бесконечномерном случае}

\section{М. Божейко, Е. В. Литвинов, И. В. Родионова}

Пусть $\nu$ - конечная мера на $\mathbb{R}$, преобразование Лапласа которой является аналитической функцией в окрестности нуля. Энионный белый шум Леви на $\left(\mathbb{R}^{d}, d x\right)$ - это некоторое семейство некоммутативных операторов $\langle\omega, \varphi\rangle$ на энионном фоковском пространстве над $L^{2}\left(\mathbb{R}^{d} \times \mathbb{R}, d x \otimes \nu\right)$. Здесь $\varphi=\varphi(x)$ - элемент пространства основных функций на $\mathbb{R}^{d}$, а $\omega=\omega(x)$ понимается как операторнозначное распределение на $\mathbb{R}^{d}$. Пусть $L^{2}(\tau)$ - некоммутативное $L^{2}$-пространство, порождаемое алгеброй многочленов от переменных $\langle\omega, \varphi\rangle$, где $\tau$ - вакуумное ожидание. Мы строим некоммутативные ортогональные многочлены в $L^{2}(\tau)$ вида $\left\langle P_{n}(\omega), f^{(n)}\right\rangle$, где $f^{(n)}$ - основная функция на $\left(\mathbb{R}^{d}\right)^{n}$. Используя эти ортогональные многочлены, мы конструируем унитарный изоморфизм $U$ между $L^{2}(\tau)$ и расширенным энионным фоковским пространством над $L^{2}\left(\mathbb{R}^{d}, d x\right)$, которое обозначается $\mathbf{F}\left(L^{2}\left(\mathbb{R}^{d}, d x\right)\right)$. Обычное энионное фоковское пространство над $L^{2}\left(\mathbb{R}^{d}, d x\right)$, обозначаемое $\mathscr{F}\left(L^{2}\left(\mathbb{R}^{d}, d x\right)\right)$, является подпространством пространства $\mathbf{F}\left(L^{2}\left(\mathbb{R}^{d}, d x\right)\right)$. Мы показываем, что равенство $\mathbf{F}\left(L^{2}\left(\mathbb{R}^{d}, d x\right)\right)=\mathscr{F}\left(L^{2}\left(\mathbb{R}^{d}, d x\right)\right)$ имеет место тогда и только тогда, когда мера $\nu$ сосредоточена в одной точке (т. е. в гауссовском или пуассоновском случае). Пользуясь унитарным изоморфизмом $U$, мы реализуем операторы $\langle\omega, \varphi\rangle$ как (трехдиагональное) поле Якоби в $\mathbf{F}\left(L^{2}\left(\mathbb{R}^{d}, d x\right)\right)$. Строится класс типа Мейкснера энионного белого шума Леви, для которого соответствующее поле Якоби в $\mathbf{F}\left(L^{2}\left(\mathbb{R}^{d}, d x\right)\right)$ имеет относительно простую структуру. Именно, каждый энионный белый шум Леви типа Мейкснера характеризуется двумя параметрами: $\lambda \in \mathbb{R}$ и $\eta \geqslant 0$. В заключение мы получаем представление $\omega(x)=\partial_{x}^{\dagger}+\lambda \partial_{x}^{\dagger} \partial_{x}+\eta \partial_{x}^{\dagger} \partial_{x} \partial_{x}+\partial_{x}$, где $\partial_{x}$ и $\partial_{x}^{\dagger}-$ операторы уничтожения и рождения в точке $x$.

Библиография: 57 названий.

Ключевые слова: энионные коммутационные соотношения, энионное фоковское пространство, гамма-процесс, поле Якоби, белый шум Леви, класс Мейкснера ортогональных многочленов.

DOI: $10.4213 / \mathrm{rm} 9668$

Работа первого и второго авторов выполнена при поддержке Польского национального научного центра (грант Dec-2012/05/B/ST1/00626) и научного центра университета г. Билефельд (SFB 701) в рамках программы "Spectral structures and topological methods in mathematics". Первый автор поддержан также программой MAESTRO (грант Dec-2011/02/A/ST1/00119).

(C) М. Божейко, Е. В. Литвинов, И. В. РодионовА, 2015 


\section{СоДЕРЖАНИЕ}

1. Многочлены Мейкснера в бесконечномерном случае.............. 76

1.1. Класс Мейкснера ортогональных многочленов ............. 76

1.2. Бесконечномерное расширение.................................. 78

1.3. Некоммутативное расширение для энионов: введение ........ 82

2. Некоммутативный белый шум Леви для энионной статистики . . . . . 83

2.1. Энионное фоковское пространство и энионные коммутационные

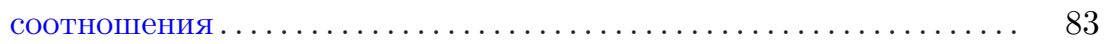

2.2. Энионный белый шум Леви и некоммутативные ортогональные

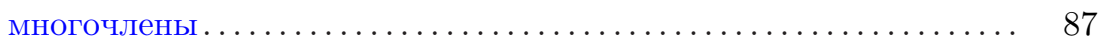

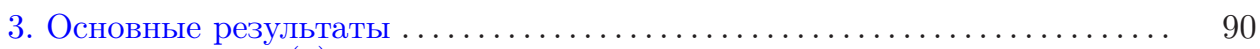

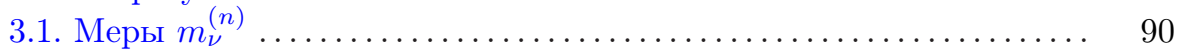

3.2. Расширенное энионное фоковское пространство ............. 91

3.3. Энионный белый шум Леви как поле Якоби .................. 93

3.4. Характеризация многочленов типа Мейкснера .............. 95

3.5. Энионный белый шум типа Мейкснера ................... 96

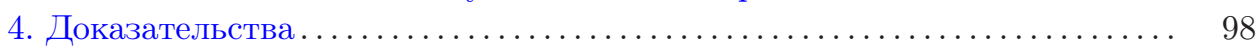

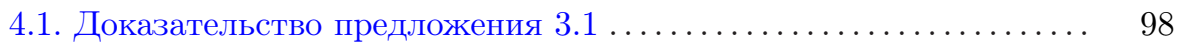

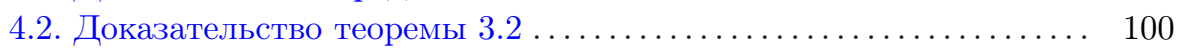

4.3. Доказательство теоремы $3.4 \ldots \ldots \ldots \ldots \ldots \ldots \ldots \ldots \ldots \ldots \ldots . \ldots \ldots$

4.4. Доказательство теоремы $3.5 \ldots \ldots \ldots \ldots \ldots \ldots \ldots \ldots \ldots \ldots \ldots . \ldots \ldots$

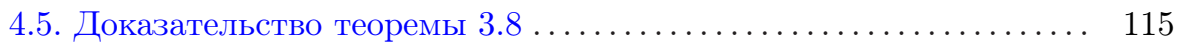

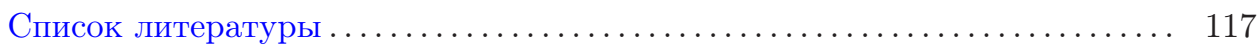

\section{1. Многочлены Мейкснера в бесконечномерном случае}

1.1. Класс Мейкснера ортогональных многочленов. В 1934 г. Мейкснер [44] рассмотрел следующую задачу. Пусть $u(z)$ и $\Phi(z)$ - комплекснозначные функции, разлагающиеся в степенной ряд по $z \in \mathbb{C}$ в окрестности нуля и такие, что $u(0)=1, \Phi(0)=0, \Phi^{\prime}(0)=1$. Тогда функция

$$
G(x, z)=\exp [x \Phi(z)] u(z)=\sum_{n=0}^{\infty} \frac{P_{n}(x)}{n !} z^{n}
$$

порождает систему приведенных многочленов $P_{n}(x)$. Возникает вопрос: как найти все многочлены такого рода, ортогональные относительно меры $\mu$ на $\mathbb{R}$ ? Такие многочлены иногда называют ортогональными многочленами с производящей функцией экспоненциального типа.

Мейкснер [44] показал, что система многочленов $P_{n}(x)$ принадлежит этому классу тогда и только тогда, когда для нее выполняется рекуррентное соотношение

$$
x P_{n}(x)=P_{n+1}(x)+(l+n \lambda) P_{n}(x)+n(k+\eta(n-1)) P_{n-1}(x), \quad n \in \mathbb{N}_{0},
$$

где $l \in \mathbb{R}, k>0, \lambda \in \mathbb{R}, \eta \geqslant 0$. Отметим, что при любом выборе параметров соответствующая мера ортогональности $\mu$ является безгранично делимой. При 
$l=0$ мера $\mu$ центрирована, а при $l \neq 0$ возникает сдвиг $\mu$ на $l$. В случае $l=0$ и $k \neq 1$ мера $\mu$ есть сверточная $k$-я степень соответствующей меры $\mu$ при $k=1$.

Имеется пять классов многочленов, для которых выполнено рекуррентное соотношение (1.2) (см. [23], [44]):

(i) если $\lambda=\eta=0$, то $\mu$ - гауссовская мера, $\left(P_{n}\right)_{n=0}^{\infty}-$ система многочленов Эрмита;

(ii) если $\lambda \neq 0, \eta=0$, то мера $\mu$ аналогична пуассоновскому распределению ( $\mu$ в точности является пуассоновским распределением при $\lambda=1$ и $l=1$ ), $\left(P_{n}\right)_{n=0}^{\infty}-$ система многочленов Шарлье;

(iii) если $|\lambda|=2 \sqrt{\eta}, \eta \neq 0$, то $\mu$ - гамма-распределение, $\left(P_{n}\right)_{n=0}^{\infty}-$ система многочленов Лагерра;

(iv) если $|\lambda|>2 \sqrt{\eta}, \eta \neq 0$, то $\mu$ - отрицательное биномиальное распределение (распределение Паскаля), $\left(P_{n}\right)_{n=0}^{\infty}-$ система многочленов Мейкснера первого рода;

(v) если $|\lambda|<2 \sqrt{\eta}, \eta \neq 0$, то $\mu$ - распределение Мейкснера, $\left(P_{n}\right)_{n=0}^{\infty}-$ система многочленов Мейкснера второго рода (или многочленов Мейкснера-Поллачека).

Отметим, что в каждом из этих случаев

$$
G(x, z)=\exp [x \Phi(z)-\mathscr{C}(\Phi(z))]
$$

где $z$ лежит в окрестности нуля в $\mathbb{C}$, а $\mathscr{C}(z):=\log \left(\int_{\mathbb{R}} e^{x z} \mu(d x)\right)$ - кумулянтное преобразование меры $\mu$. По поводу явных формул для $\Phi(z)$ и $\mathscr{C}(z)$ мы отсылаем читателя к [23], [44]. Если ввести комплексные параметры $\alpha, \beta \in \mathbb{C}$ такие, что $\alpha+\beta=-\lambda, \alpha \beta=\eta$, и использовать разложение в ряд Тейлора, то $\Phi(z)$ и $\mathscr{C}(z)$ можно записать в явном и единообразном виде для всех параметров $\alpha, \beta$ (см. [47]).

Для дальнейших рассмотрений ключевыми являются следующие два факта. Во-первых, полагая $l=0, k=1$, мы можем переписать соотношение (1.2) в следующем виде:

$$
x=\partial^{\dagger}+\lambda \partial^{\dagger} \partial+\partial+\eta \partial^{\dagger} \partial \partial .
$$

Здесь (мы позволяем себе некоторую вольность в обозначениях) $x$ - оператор умножения на переменную $x$ из $L^{2}(\mathbb{R}, \mu)$, $\partial^{\dagger}$ - оператор рождения (повышающий оператор), т. е. $\partial^{\dagger} P_{n}(x)=P_{n+1}(x)$, а $\partial$ - оператор уничтожения (понижающий оператор), т. е. $\partial P_{n}(x)=n P_{n-1}(x)$.

Во-вторых, колмогоровское представление преобразования Фурье безгранично делимой меры $\mu$ (при $l=0)$ имеет следующий вид [48], [49]:

$$
\int_{\mathbb{R}} e^{i u x} \mu(d x)=\exp \left[k \int_{\mathbb{R}}\left(e^{i u s}-1-i u s\right) s^{-2} \nu(d s)\right], \quad u \in \mathbb{R}
$$

(см. также [30]). Здесь при $\eta=0$ (гауссовский и пуассоновский случаи) $\nu=\delta_{\lambda}-$ мера Дирака с массой, сосредоточенной в точке $\lambda$, а при $\eta \neq 0$ (случаи (iii)-(v)) $\nu$ - вероятностная мера на $\mathbb{R}$, для которой соответствующая система приведенных ортогональных многочленов $\left(p_{n}\right)_{n=0}^{\infty}$ Удовлетворяет рекуррентной формуле

$$
s p_{n}(s)=p_{n+1}(s)+\lambda(n+1) s+\eta n(n+1) p_{n-1}(s) .
$$


В частности, $\left(p_{n}\right)_{n=0}^{\infty}$ снова является системой ортогональных многочленов из класса Мейкснера.

1.2. Бесконечномерное расширение. Оказывается, что класс Мейкснера ортогональных многочленов имеет фундаментальное значение в бесконечномерном анализе и, в частности, в теории белого шума Леви (см., например, [1], [41], [42], [48], [52] и приведенные там ссылки). Пусть $X:=\mathbb{R}^{d}$, и пусть

$$
\mathscr{D}(X) \subset L^{2}(X, d x) \subset \mathscr{D}^{\prime}(X)
$$

- стандартная тройка пространств, в которой $\mathscr{D}(X)$ - ядерное пространство гладких функций на $X$ с компактным носителем, а $\mathscr{D}^{\prime}(X)$ - двойственное пространство к $\mathscr{D}(X)$ относительно нулевого пространства $L^{2}(X, d x)$. При $\omega \in \mathscr{D}^{\prime}(X)$ и $\varphi \in \mathscr{D}(X)$ обозначим через $\langle\omega, \varphi\rangle$ отношение двойственности для $\omega$ и $\varphi$. Пусть $\mu$ - вероятностная мера на $\mathscr{D}^{\prime}(X)$. Предположим, что $\mu$ - обобщенный стохастический процесс с независимыми значениями (в смысле [27]), или, в другой терминологии, белошумовая мера Леви (Lévy white noise measure) [25]. Мы также предположим, что мера $\mu$ центрирована и ее преобразование Фурье имеет колмогоровское представление

$$
\int_{\mathscr{D}^{\prime}(X)} e^{i\langle\omega, \varphi\rangle} \mu(d \omega)=\exp \left[\int_{X} \int_{\mathbb{R}}\left(e^{i s \varphi(x)}-1-i s \varphi(x)\right) s^{-2} \nu(d s) d x\right], \quad \varphi \in \mathscr{D}(X),
$$

где $\nu$ - вероятностная мера на $\mathbb{R}$ такая, что

$$
\int_{\mathbb{R}} e^{\varepsilon|s|} \nu(d s)<\infty \quad \text { при некотором } \varepsilon>0 .
$$

Meра $s^{-2} \nu(d s)$ на $\mathbb{R} \backslash\{0\}$ называется мерой Леви процесса $\mu$, при этом $\nu(\{0\})$ описывает гауссовскую часть процесса $\mu$ (при $s=0$ подынтегральное выражение в (1.6) равно $\left.-(1 / 2) \varphi^{2}(x)\right)$.

В случае $d=1$ для каждого $t \geqslant 0$ с помощью приближений в $L^{2}\left(\mathscr{D}^{\prime}(X), \mu\right)$ можно определить случайную величину $L_{t}(\omega)=\left\langle\omega, \chi_{[0, t]}\right\rangle$. Здесь $\chi_{[0, t]}-$ характеристическая функция интервала $[0, t]$. Тогда $\left(L_{t}\right)_{t \geqslant 0}-$ (версия) процесса Леви с колмогоровской мерой $\nu$ :

$$
\int_{\mathscr{D}^{\prime}(X)} e^{i u L_{t}(\omega)} \mu(d \omega)=\exp \left[t \int_{\mathbb{R}}\left(e^{i u s}-1-i u s\right) s^{-2} \nu(d s)\right] .
$$

Таким образом, мера $\mu$ в действительности является белошумовой мерой Леви.

Через $\mathscr{C} \mathscr{P}$ обозначим множество всех непрерывных многочленов на $\mathscr{D}^{\prime}(X)$, т. е. функций на $\mathscr{D}^{\prime}(X)$ вида

$$
\begin{gathered}
f^{(0)}+\sum_{i=1}^{n}\left\langle\omega^{\otimes i}, f^{(i)}\right\rangle, \quad \omega \in \mathscr{D}^{\prime}(X), \\
f^{(0)} \in \mathbb{R}, \quad f^{(i)} \in \mathscr{D}(X)^{\otimes i}, \quad i=1, \ldots, n, \quad n \in \mathbb{N} .
\end{gathered}
$$

Если $f^{(n)} \neq 0$, то по определению многочлен из (1.8) имеет порядок $n$. Множество $\mathscr{C} \mathscr{P}$ плотно в $L^{2}\left(\mathscr{D}^{\prime}(X), \mu\right)$. Многочлены из $\mathscr{C} \mathscr{P}$ можно ортогонализовать, используя подход, предложенный А.В. Скороходом [50]. Более точно, 
обозначим через $\mathscr{C} \mathscr{P}_{n}$ линейное пространство всех непрерывных многочленов на $\mathscr{D}^{\prime}(X)$ порядка $\leqslant n$. Пусть $\mathscr{M} \mathscr{P}_{n}-$ замыкание $\mathscr{C} \mathscr{P}_{n}$ в $L^{2}\left(\mathscr{D}^{\prime}(X), \mu\right)$ (множество измеримых многочленов порядка $\leqslant n$ ). Далее, пусть $\mathscr{O} \mathscr{P}_{n}:=$ $\mathscr{M} \mathscr{P}_{n} \ominus \mathscr{M} \mathscr{P}_{n-1}-$ множество ортогонализованных многочленов на $\mathscr{D}^{\prime}(X)$ порядка $n$. Тогда несложно видеть, что

$$
L^{2}\left(\mathscr{D}^{\prime}(X), \mu\right)=\bigoplus_{n=0}^{\infty} \mathscr{O} \mathscr{P}_{n}
$$

ЗАмечАниЕ 1.1. Альтернативное ортогональное разложение $L^{2}$-пространства, соответствующее процессу Леви, было предложено А. М. Вершиком и Н. В. Цилевич [57].

Для каждого $f^{(n)} \in \mathscr{D}(X)^{\otimes n}$ обозначим через $\left\langle P_{n}(\omega), f^{(n)}\right\rangle$ ортогональную проекцию непрерывного монома $\left\langle\omega^{\otimes n}, f^{(n)}\right\rangle$ на $\mathscr{O} \mathscr{P}_{n}$. Через $\mathscr{O} \mathscr{C} \mathscr{P}$ обозначим линейное пространство ортогонализованных непрерывных многочленов, т. е. пространство конечных сумм функций вида $\left\langle P_{n}(\omega), f^{(n)}\right\rangle$ и констант. Отметим, что функция $\left\langle P_{n}(\omega), f^{(n)}\right\rangle$ не обязана содержаться в $\mathscr{C} \mathscr{P}$.

Теорема 1.2. Пусть $\mu$ - вероятностная мера на $\mathscr{D}^{\prime}(X)$, имеющая преобразование Фуръе (1.6), где $\nu$ - вероятностная мера на $\mathbb{R}$, удовлетворяющая условию (1.7). В этом случае равенство

$$
\mathscr{C} \mathscr{P}=\mathscr{O} \mathscr{C} \mathscr{P}
$$

имеет место тогда и только тогда, когда существуют $\lambda \in \mathbb{R} u \eta \geqslant 0$ такие, что если $\eta=0$, то $\nu=\delta_{\lambda}$, а если $\eta>0$, то система приведенньх

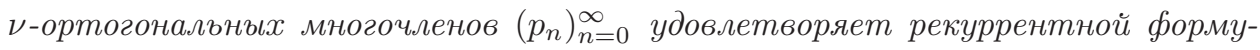
ле (1.5) с указанными $\lambda$ и $\eta$.

Данную теорему можно вывести из основного результата работы [9]. Она также является следствием теоремы 3.5, которую мы сформулируем ниже.

Определим производящую функцию семейства ортогональных многочленов:

$$
G_{\mu}(\omega, \varphi):=\sum_{n=0}^{\infty} \frac{1}{n !}\left\langle P_{n}(\omega), \varphi^{\otimes n}\right\rangle
$$

и рассмотрим кумулянтное преобразование меры $\mu$ :

$$
\mathscr{C}_{\mu}(\varphi):=\log \left(\int_{\mathscr{D}^{\prime}(X)} e^{\langle\omega, \varphi\rangle} \mu(d \omega)\right) .
$$

В следующей теореме показывается, в частности, что формулу (1.3) можно распространить на бесконечномерный случай (по поводу доказательства мы отсылаем читателя к [41]).

Теорема 1.3. Пусть $\lambda \in \mathbb{R} u \eta \geqslant 0$ фиксированы, и пусть $\mu$ - вероятностная мера на $\mathscr{D}^{\prime}(X)$, преобразование Фуръе которой имеет колмогоровское представление (1.6), где $\nu$ - вероятностная мера на $\mathbb{R}$ с параметрами $\lambda u \eta$, 
как в теореме 1.2. Далее, пусть $\mathscr{C}(\cdot)$ и $\Phi(\cdot)$ - функиии из (1.3) с определенными выше параметрами $l=0, k=1, \lambda u \eta$. Тогда

$$
\begin{aligned}
\mathscr{C}_{\mu}(\varphi) & =\int_{X} \mathscr{C}(\varphi(x)) d x \\
G_{\mu}(\omega, \varphi) & =\exp \left[\langle\omega, \Phi(\varphi)\rangle-\int_{X} \mathscr{C}(\Phi(\varphi(x))) d x\right],
\end{aligned}
$$

где ч может быть взято (во всяком случае) из окрестности нуля в $\mathscr{D}(X)$.

В случае $\lambda=0, \eta=0$ мера $\mu$ является гауссовской белошумовой мерой. (По поводу теории гауссовского белого шума мы отсылаем читателя, например, к монографиям [8], [25], [33].)

В случае $\lambda \neq 0, \eta=0$ мера $\mu$ является пуассоновской случайной мерой (точечным процессом) - см., например, [36]. Представления групп диффеоморфизмов в пространстве Пуассона рассмотрены в работе [55], по поводу анализа пуассоновского белого шума мы отсылаем читателя к работе [35], а по поводу пуассоновского анализа на конфигурационном пространстве - к работе [2].

При $\eta \neq 0$ наиболее важным является случай меры $\mu$ при $\lambda=2$ и $\eta=1$. Тогда $\mu$ - центрированная гамма-мера. Известно, что гамма-мера сосредоточена на конусе дискретных мер Радона на $X, \sum_{i} s_{i} \delta_{x_{i}}$, для которых конфигурация атомов $\left\{x_{i}\right\}$ составляет плотное подмножество в $X$. Чрезвычайно важным свойством гамма-меры является то, что она квазиинвариантна относительно действия естественной группы преобразований весов $s_{i}$ (см. [52] и приведенные там ссылки). Более того, в [52] показано, что гамма-мера является единственным законом распределения мерозначного процесса Леви с эквивалентной $\sigma$-конечной мерой, являющейся проективно-инвариантной относительно естественной группы преобразований весов $s_{i}$. В [52] такая $\sigma$-конечная мера называется бесконечномерной лебеговой мерой (см. также [53]). Кроме того, отметим, что в работах [26], [52], [54], [56] гамма-мера использовалась в теории представлений групп вида $S L(2, F)$, где $F$ - алгебра функций на многообразии. Впервые анализ белого шума, соответствующего гамма-мере, был выполнен в [37]. Этот вопрос получил дальнейшее развитие в [38]. Гиббсовские возмущения гамма-меры были построены в [32]. Оператор Лапласа, соответствующий гамма-мере, был построен и изучен в [31]. Вопросы бесконечномерного анализа в общем случае $\eta \neq 0$ рассматривались в [40], [41].

Хорошо известно, что в гауссовском и пуассоновском случаях $(\eta=0)$ разложение $L^{2}\left(\mathscr{D}^{\prime}(X), \mu\right)$ по ортогональным многочленам влечет существование изоморфизма Винера-Ито-Сигала между $L^{2}\left(\mathscr{D}^{\prime}(X), \mu\right)$ и симметричным фоковским пространством над $L^{2}(X, d x)$ (этот результат может быть получен и иным путем - с использованием кратных стохастических интегралов, см., например, [51] по поводу пуассоновского случая.) Данный результат может быть усилен следующим образом (см. [37], [38], [41]).

Теорема 1.4. Пусть $\lambda \in \mathbb{R}, \eta \geqslant 0$, и пусть $\mu$ - соответствующая вероятностная мера на $\mathscr{D}^{\prime}(X)$, определенная в теореме 1.3 . 
(i) Для каждого $n \in \mathbb{N}$ существует мера $m_{\nu}^{(n)}$ на $X^{n}$ такая, что

$$
\int_{\mathscr{D}^{\prime}(X)}\left\langle P_{n}(\omega), f^{(n)}\right\rangle^{2} \mu(d \omega)=\int_{X^{n}}\left(\operatorname{Sym}_{n} f^{(n)}\right)^{2} d m_{\nu}^{(n)}, \quad f^{(n)} \in \mathscr{D}(X)^{\otimes n},
$$

где $\operatorname{Sym}_{n} f^{(n)}$ - обычная симметризация функиии $f^{(n)}$ и $m_{\nu}^{(n)}=\frac{1}{n !} d x_{1} \cdots d x_{n}$ при $\eta=0$ (при $\eta \neq 0$ точная конструкиия меры $m_{\nu}^{(n)}$ дается в $\left.n .3 .1\right)$.

(ii) Определим гильбертово пространство

$$
\mathbf{F}_{\mathrm{sym}}\left(L^{2}(X, d x), \nu\right):=\mathbb{R} \oplus \bigoplus_{n=1}^{\infty} L_{\mathrm{sym}}^{2}\left(X^{n}, m_{\nu}^{(n)}\right),
$$

где $L_{\mathrm{sym}}^{2}\left(X^{n}, m_{\nu}^{(n)}\right)$ - подпространство $L^{2}\left(X^{n}, m_{\nu}^{(n)}\right)$, состоящее из всех симметричных функций. Если $\eta=0$, то $\mathbf{F}_{\mathrm{sym}}\left(L^{2}(X, d x), \nu\right)$ - симметричное фоковское пространство над $L^{2}(X, d x)$. Если $\eta \neq 0, \operatorname{mo} \mathbf{F}_{\mathrm{sym}}\left(L^{2}(X, d x), \nu\right)$ содержит симметричное фоковское пространство в качестве собственного подпространства. Мы будем называть $\mathbf{F}_{\text {sуm }}\left(L^{2}(X, d x), \nu\right)$ расширенным симметричным фоковским пространством. Отображение

$$
\begin{aligned}
f^{(0)} & +\sum_{i=i}^{n}\left\langle P_{i}(\omega), f^{(i)}\right\rangle \\
& \mapsto\left(f^{(0)}, \operatorname{Sym}_{1} f^{(1)}, \ldots, \operatorname{Sym}_{n} f^{(n)}, 0,0, \ldots\right) \in \mathbf{F}_{\mathrm{sym}}\left(L^{2}(X, d x), \nu\right)
\end{aligned}
$$

продолжается по непрерывности до унитарного оператора $U: L^{2}\left(\mathscr{D}^{\prime}(X), \mu\right) \rightarrow$ $\mathbf{F}_{\text {sym }}\left(L^{2}(X, d x), \nu\right)$.

(iii) Для каждого $\varphi \in \mathscr{D}(X)$ мы сохраняем обозначение $\langle\omega, \varphi\rangle$ для образа оператора умножения на моном $\langle\omega, \varphi\rangle$ в $L^{2}\left(\mathscr{D}^{\prime}(X), \mu\right)$ под действием унитарного оператора $U$. Тогда, аналогично (1.4), имеет место представление оператора $\langle\omega, \varphi\rangle$, реализованного в (расширенном) симметричном фоковском пространстве $\mathbf{F}_{\mathrm{sym}}\left(L^{2}(X, d x), \nu\right)$ :

$$
\langle\omega, \varphi\rangle=\int_{X} d x \varphi(x)\left(\partial_{x}^{\dagger}+\lambda \partial_{x}^{\dagger} \partial_{x}+\partial_{x}+\eta \partial_{x}^{\dagger} \partial_{x} \partial_{x}\right)
$$

Здесь $\partial_{x}$ - оператор уничтожения в точке $x$ :

$$
\left(\partial_{x} f^{(n)}\right)\left(x_{1}, \ldots, x_{n-1}\right):=n f^{(n)}\left(x, x_{1}, \ldots, x_{n-1}\right),
$$

a $\partial_{x}^{\dagger}$ - оператор рождения в точке $x$, задаваемый формулой

$$
\int_{X} d x \varphi(x) \partial_{x}^{\dagger} f^{(n)}:=\operatorname{Sym}_{n+1}\left(\varphi \otimes f^{(n)}\right)
$$

см. [41] по поводу далънейших деталей.

Отметим, что ввиду формулы (1.13) мы можем эвристически записать

$$
\omega(x)=\partial_{x}^{\dagger}+\lambda \partial_{x}^{\dagger} \partial_{x}+\partial_{x}+\eta \partial_{x}^{\dagger} \partial_{x} \partial_{x} .
$$

Из теоремы 1.4 , (iii) следует, что при реализации в $\mathbf{F}_{\text {sym }}\left(L^{2}(X, d x), \nu\right)$ операторы $\langle\omega, \varphi\rangle$ образуют поле Якоби, т. е. они имеют тридиагональную структуру (ср., например, с [7], [9], [20], [21], [40]). 
1.3. Некоммутативное расширение для энионов: введение. Результаты, рассмотренные выше, имеют некоммутативные аналоги в свободной теории вероятностей [16], [17] (см. также [4], [5], [10], [12], [13], [43] и приведенные там ссылки). По поводу дальнейших связей между классическими распределениями из класса Мейкснера и свободной теорией вероятностей см. [6], [14].

В настоящей работе мы будем интересоваться некоммутативным расширением многочленов Мейкснера для так называемой энионной статистики [39], [28], [29] (см. также [11]). Энионная статистика, индексируемая комплексными числами $q$ с единичным модулем, образует непрерывный мостик между бозонной статистикой $(q=1)$ и статистикой Ферми $(q=-1)$. Одной из основных целей настоящей работы является демонстрация того, что в энионном случае естественно возникают некоммутативные многочлены типа Мейкснера, имеющие представление типа (1.13).

По существу, вера в существование аналога формулы (1.13) в фермионном случае могла бы показаться бесперспективной. Действительно, если операторы $\partial_{x}$ и $\partial_{y}$ антикоммутируют, т. е. $\partial_{x} \partial_{y}=-\partial_{y} \partial_{x}$, то $\partial_{x} \partial_{x}=0$ и, стало быть, член $\eta \partial_{x}^{\dagger} \partial_{x} \partial_{x}$ обязан быть равным нулю. Однако мы показываем, что даже в фермионном случае интегрирование $\int_{X} d x \varphi(x) \partial_{x}^{\dagger} \partial_{x} \partial_{x}$ приводит к нетривиальному оператору в расширенном антисимметричном фоковском пространстве $\mathbf{F}_{\text {as }}\left(L^{2}(X, d x), \nu\right)$. Такое пространство содержит классическое антисимметричное (фермионное) фоковское пространство $\mathscr{F}_{\text {as }}\left(L^{2}(X, d x)\right)$ в качестве подпространства. На пространстве $\mathscr{F}_{\text {as }}\left(L^{2}(X, d x)\right)$ операторы $\partial_{x}$ и $\partial_{y}$ в действительности антикоммутируют. Однако такая антикоммутация уже не имеет места на всем пространстве $\mathbf{F}_{\text {as }}\left(L^{2}(X, d x), \nu\right)$. Как результат, использование расширенного антисимметричного фоковского пространства приводит к некоторой перенормировке (а точнее, к нетривиальному расширению) операторов $\partial_{x}$ и $\partial_{x}^{\dagger}$.

Наше рассмотрение некоммутативного расширения будет построено следующим образом. Следуя [39], [18], [28], в разделе 2 мы схематично напоминаем конструкцию энионного фоковского пространства и определяем на нем операторы и энионные коммутационные соотношения. Мы также напоминаем конструкцию белого шума Леви для энионной статистики, который строится как семейство некоммутативных самосопряженных операторов $\langle\omega, \varphi\rangle$ в энионном фоковском пространстве над $L^{2}(X \times \mathbb{R}, d x \nu(d s))$ (см. [18] по поводу дальнейших подробностей). Отметим, что в разделе 2 мы не объясняем, почему "приращения" такого процесса могут пониматься как "энионно-независимые", отсылая в этой связи читателя к работе [18]. Мы только отметим, что в коммутативном, бозонном, случае $(q=1)$ мы в действительности можем восстановить классический белый шум Леви, реализуемый как семейство коммутирующих самосопряженных операторов в симметричном фоковском пространстве над $L^{2}(X \times \mathbb{R}, d x \nu(d s))$.

Основные результаты работы формулируются в разделе 3 . В частности, отталкиваясь от пространства $\mathscr{C} \mathscr{P}$ некоммутативных непрерывных многочленов от энионного белого шума, мы строим пространство $\mathscr{O} \mathscr{C} \mathscr{P}$ ортогонализованных непрерывных многочленов. По аналогии с (1.10) для каждого $n \in \mathbb{N}$ 
строится мера $m_{\nu}^{(n)}$ на $X^{n}$ и находится соответствующий оператор симметризации $\mathrm{Sym}_{n}$. Исходя из такой симметрии мы находим нетривиальное расширение для энионной симметрии (в частности, для симметрии фермионов). После этого, следуя (1.11), мы определяем расширенное энионное фоковское пространство и далее, по аналогии с (1.12), строим унитарный оператор $U$, отображающий некоммутативное $L^{2}$-пространство в расширенное энионное фоковское пространство. Под действием унитарного оператора $U$ каждый оператор $\langle\omega, \varphi\rangle$ выражается в форме Якоби в расширенном энионном фоковском пространстве. Мы показываем, что такое поле Якоби принимает наиболее простой (в определенном смысле) вид, если $\nu$ является мерой, указанной в теореме 1.2, т. е. $\nu$ - колмогоровская мера белошумовой меры $\mu$ из класса Мейкснера. Отметим, что в этом случае имеют место аналоги формул (1.13)-(1.15).

Доказательства основных результатов даются в разделе 4.

Среди ряда открытых проблем, касающихся энионного белого шума типа Мейкснера, упомянем только две.

(i) Как в классическом, так и в свободном случае производящие функции ортогональных многочленов типа Мейкснера известны в явном виде и играют важную роль при изучении таких многочленов. В энионном случае форма производящей функции на данный момент не известна даже в гауссовском случае. Здесь основная трудность заключается в том, что как классическим, так и свободным многочленам типа Мейкснера соответствуют системы ортогональных многочленов на действительной прямой. Однако энионный случай является чисто бесконечномерным и не имеет связи с соответствующей одномерной теорией.

(ii) В [1] показано, что в классическом случае процессы Леви из класса Мейкснера с $\eta>0$ связаны с перенормированными квадратами бозонного белого шума. Возможно ли интерпретировать энионные белые шумы типа Мейкснера как белые шумы, связанные с перенормированными квадратами энионного белого шума?

\section{2. Некоммутативный белый шум Леви для энионной статистики}

2.1. Энионное фоковское пространство и энионные коммутационные соотношения. Пусть $\mathscr{B}(X)$ - борелевская $\sigma$-алгебра на $X$, а $\mathscr{B}_{0}(X)$ подкласс множеств из $\mathscr{B}(X)$, имеющих компактное замыкание. Через $m=$ $m(d x)=d x$ мы обозначим меру Лебега на $(X, \mathscr{B}(X))$.

Для каждого $n \geqslant 2$ определим

$$
X^{(n)}:=\left\{\left(x_{1}, \ldots, x_{n}\right) \in X^{n} \mid x_{i} \neq x_{j} \forall 1 \leqslant i<j \leqslant n\right\} .
$$

Так как мера $m$ неатомарна, то

$$
m^{\otimes n}\left(X \backslash X^{(n)}\right)=0 .
$$

На $X$ введем строгий линейный порядок следующим образом. Если $x=$ $\left(x^{1}, \ldots, x^{d}\right) \in X$ и $y=\left(y^{1}, \ldots, y^{d}\right) \in X, x \neq y$, то мы считаем, что $x<y$, если $x^{1}=y^{1}, \ldots, x^{j-1}=y^{j-1}$ и $x^{j}<y^{j}$ при некотором $j \in\{1, \ldots, d\}$. 
Зафиксируем число $q \in \mathbb{C},|q|=1$, и определим функцию $Q: X^{(2)} \rightarrow \mathbb{C}$ следующим образом:

$$
Q(x, y)= \begin{cases}q, & \text { если } x<y, \\ \bar{q}, & \text { если } y<x .\end{cases}
$$

Отметим, что функция $Q$ является эрмитовой:

$$
Q(x, y)=\overline{Q(y, x)}, \quad(x, y) \in X^{(2)} .
$$

Функция $f^{(n)}: X^{(n)} \rightarrow \mathbb{C}(n \geqslant 2)$ называется $Q$-симметричной, если для каждого $j=1, \ldots, n-1$

$$
f^{(n)}\left(x_{1}, \ldots, x_{n}\right)=Q\left(x_{j}, x_{j+1}\right) f^{(n)}\left(x_{1}, \ldots, x_{j-1}, x_{j+1}, x_{j}, x_{j+2}, \ldots, x_{n}\right) .
$$

Через $\mathscr{H}:=L^{2}(X, m)$ обозначим гильбертово пространство комплекснозначных функций на $X$, интегрируемых с квадратом. Тогда $\mathscr{H}{ }^{\otimes n}=L^{2}\left(X^{n}, m^{\otimes n}\right)$ для каждого $n \in \mathbb{N}$. С учетом $(2.2)$ имеем $\mathscr{H}^{\otimes n}=L^{2}\left(X^{(n)}, m^{\otimes n}\right)$. Определим комплексное гильбертово пространство $\mathscr{H}^{\circledast n}$ как (замкнутое) подпространство из $\mathscr{H}^{\otimes n}$, состоящее из всех $\left(m^{\otimes n}\right.$-версий) $Q$-симметричных функций из $\mathscr{H}^{\otimes n}$. Через $\operatorname{Sym}_{n}$ обозначим ортогональную проекцию $\mathscr{H}^{\otimes n}$ на $\mathscr{H}^{\circledast n}$. Этот оператор имеет следующее явное представление: для каждой $f^{(n)} \in \mathscr{H}^{\otimes n}$

$$
\begin{aligned}
\left(\operatorname{Sym}_{n} f^{(n)}\right)\left(x_{1}, \ldots, x_{n}\right)=\frac{1}{n !} & \sum_{\pi \in \mathfrak{S}_{n}} Q_{\pi}\left(x_{1}, \ldots, x_{n}\right) \\
& \times f^{(n)}\left(x_{\pi^{-1}(1)}, \ldots, x_{\pi^{-1}(n)}\right), \quad\left(x_{1}, \ldots, x_{n}\right) \in X^{(n)} .
\end{aligned}
$$

Здесь $\mathfrak{S}_{n}-$ группа всех перестановок элементов $1, \ldots, n$ и

$$
Q_{\pi}\left(x_{1}, \ldots, x_{n}\right):=\prod_{\substack{1 \leqslant i<j \leqslant n \\ \pi(i)>\pi(j)}} Q\left(x_{i}, x_{j}\right), \quad\left(x_{1}, \ldots, x_{n}\right) \in X^{(n)} .
$$

Теперь мы можем определить $Q$-симметричное тензорное произведение $\circledast$. Именно, для $m, n \in \mathbb{N}$ и $f^{(m)} \in \mathscr{H}^{\circledast m}, g^{(n)} \in \mathscr{H}^{\circledast n}$ мы полагаем

$$
f^{(m)} \circledast g^{(n)}:=\operatorname{Sym}_{m+n}\left(f^{(m)} \otimes g^{(n)}\right) .
$$

Данное тензорное произведение ассоциативно. Отметим, что при $q=1$ произведение $\circledast$ является классическим симметричным тензорным произведением, а при $q=-1$ - классическим антисимметричным произведением.

Далее, определим энионное фоковское пространство следующим образом:

$$
\mathscr{F}^{Q}(\mathscr{H}):=\bigoplus_{n=0}^{\infty} \mathscr{H}^{\circledast n} n !
$$

Тогда $\mathscr{F}^{Q}(\mathscr{H})$ - гильбертово пространство, состоящее из всех последовательностей вида $F=\left(f^{(0)}, f^{(1)}, f^{(2)}, \ldots\right)$, где $f^{(n)}$ принадлежат $\mathscr{H}^{\circledast n}\left(\mathscr{H}^{\circledast 0}:=\mathbb{C}\right)$ и удовлетворяют условию

$$
\|F\|_{\mathscr{F} Q(\mathscr{H})}^{2}:=\sum_{n=0}^{\infty}\left\|f^{(n)}\right\|_{\mathscr{H} \circledast n}^{2} n !<\infty .
$$


(Скалярное произведение в $\mathscr{F}^{Q}(\mathscr{H})$ порождается нормой пространства.) Вектор $\Omega:=(1,0,0, \ldots) \in \mathscr{F}^{Q}(\mathscr{H})$ называется вакуумным. Через $\mathscr{F}_{\text {fin }}^{Q}(\mathscr{H})$ обозначим подпространство пространства $\mathscr{F}^{Q}(\mathscr{H})$, состоящее их всех конечных последовательностей

$$
F=\left(f^{(0)}, f^{(1)}, \ldots, f^{(n)}, 0,0, \ldots\right),
$$

в которых $f^{(i)} \in \mathscr{H}^{\circledast i}$ при $i=0,1, \ldots, n, n \in \mathbb{N}$. На таком пространстве можно ввести топологию топологической прямой суммы пространств $\mathscr{H}^{\circledast n}$. Тогда сходимость в $\mathscr{F}_{\text {fin }}^{Q}(\mathscr{H})$ означает равномерную конечность ненулевых компонент вкупе с покоординатной сходимостью в $\mathscr{H}^{\circledast n}$.

Для каждого $h \in \mathscr{H}$ определим оператор рождения $a^{+}(h)$ и оператор уничтожения $a^{-}(h)$ как линейные операторы на $\mathscr{F}_{\text {fin }}^{Q}(\mathscr{H})$, заданные следующим образом:

$$
a^{+}(h) f^{(n)}:=h \circledast f^{(n)}, \quad f^{(n)} \in \mathscr{H}^{\circledast n}, \quad a^{-}(h):=a^{+}(h)^{*}\left\lceil\mathscr{F}_{\text {fin }}^{Q}(\mathscr{H}) .\right.
$$

Оба оператора $a^{+}(h)$ и $a^{-}(h)$ действуют непрерывно на $\mathscr{F}_{\text {fin }}^{Q}(\mathscr{H})$. Действительно, при $h \in \mathscr{H}$ и $f^{(n)} \in \mathscr{H}^{\circledast n}$ имеем

$$
\begin{aligned}
\left(a^{+}(h) f^{(n)}\right)\left(x_{1}, \ldots, x_{n+1}\right)= & \frac{1}{n+1}\left[h\left(x_{1}\right) f^{(n)}\left(x_{2}, \ldots, x_{n+1}\right)\right. \\
& +\sum_{k=2}^{n+1} Q\left(x_{1}, x_{k}\right) Q\left(x_{2}, x_{k}\right) \cdots Q\left(x_{k-1}, x_{k}\right) \\
& \left.\times h\left(x_{k}\right) f^{(n)}\left(x_{1}, \ldots, x_{k-1}, x_{k+1}, \ldots, x_{n+1}\right)\right], \\
\left(a^{-}(h) f^{(n)}\right)\left(x_{1}, \ldots, x_{n-1}\right)= & n \int_{X} \overline{h(y)} f^{(n)}\left(y, x_{1}, \ldots, x_{n-1}\right) d y
\end{aligned}
$$

Действие оператора уничтожения также может быть записано следующим образом: при любых $h \in \mathscr{H}$ и $f^{(n)} \in \mathscr{H}^{\otimes n}$

$$
\begin{aligned}
& \left(a^{-}(h) \operatorname{Sym}_{n} f^{(n)}\right)\left(x_{1}, \ldots, x_{n-1}\right)=\operatorname{Sym}_{n-1}\left(\int _ { X } \overline { h ( y ) } \left[\sum_{k=1}^{n} Q\left(y, x_{1}\right) Q\left(y, x_{2}\right) \times \cdots\right.\right. \\
& \left.\left.\quad \times Q\left(y, x_{k-1}\right) f^{(n)}\left(x_{1}, x_{2}, \ldots, x_{k-1}, y, x_{k}, \ldots, x_{n-1}\right)\right] d y\right)
\end{aligned}
$$

Теперь рассмотрим операторы рождения и уничтожения в точках пространства $X$. Для каждого $x \in X$ мы можем рассмотреть, по крайней мере неформально, дельта-функцию $\delta_{x}$ в точке $x$. Полагая эвристически $\partial_{x}^{\dagger}:=a^{+}\left(\delta_{x}\right)$, $\partial_{x}:=a^{-}\left(\delta_{x}\right)$, имеем

$$
\partial_{x}^{\dagger} f^{(n)}=\delta_{x} \circledast f^{(n)}, \quad \partial_{x} f^{(n)}:=n f^{(n)}(x, \cdot) .
$$

Таким образом,

$$
a^{+}(h):=\int_{X} d x h(x) \partial_{x}^{\dagger}, \quad a^{-}(h)=\int_{X} d x \overline{h(x)} \partial_{x} .
$$


Отметим, что вторая формула в $(2.8)$ дает строгое определение $\partial_{x}$ (для $m$-почти всех $x \in X)$, в то время как первая формула в (2.9) есть строгое определение интеграла $\int_{X} d x h(x) \partial_{x}^{\dagger}$.

Пусть $B_{0}\left(X^{n}\right)$ - пространство всех комплекснозначных ограниченных измеримых функций на $X^{n}$ с компактным носителем. Зафиксируем $g^{(n)} \in B_{0}\left(X^{n}\right)$ и рассмотрим произвольную последовательность $\left(\sharp_{1}, \ldots, \sharp_{n}\right)$ длины $n \geqslant 2$, состоящую из + и -. Несложно видеть, что выражение

$$
\int_{X^{n}} d x_{1} \cdots d x_{n} g^{(n)}\left(x_{1}, \ldots, x_{n}\right) \partial_{x_{1}}^{\sharp_{1}} \cdots \partial_{x_{n}}^{\sharp_{n}}
$$

определяет линейный непрерывный оператор на $\mathscr{F}_{\text {fin }}^{Q}(\mathscr{H})$. Здесь мы использовали обозначения $\partial_{x}^{+}:=\partial_{x}^{\dagger}, \partial_{x}^{-}:=\partial_{x}$.

Операторы рождения и уничтожения удовлетворяют энионным коммутационным соотношениям:

$$
\begin{aligned}
\partial_{x} \partial_{y}^{\dagger} & =\delta(x, y)+Q(x, y) \partial_{y}^{\dagger} \partial_{x}, \\
\partial_{x} \partial_{y} & =Q(y, x) \partial_{y} \partial_{x} \\
\partial_{x}^{\dagger} \partial_{y}^{\dagger} & =Q(y, x) \partial_{y}^{\dagger} \partial_{x}^{\dagger},
\end{aligned}
$$

где $\delta(x, y)$ понимается в следующем смысле:

$$
\int_{X^{2}} d x d y g^{(2)}(x, y) \delta(x, y):=\int_{X} d x g^{(2)}(x, x)
$$

Формулы (2.10)-(2.12) приобретают строгий смысл после сглаживания с функциями $g^{(2)} \in B_{0}\left(X^{2}\right)$. Отметим, что при $q=1$ выражения (2.10)-(2.12) становятся каноническими коммутационными соотношениями, а при $q=-1-$ каноническими антикоммутационными соотношениями.

ЗАмечание 2.1. Пусть $D:=\{(x, x) \mid x \in X\}$. Отметим, что для каждого $g^{(2)} \in B_{0}\left(X^{2}\right)$ с носителем в $D$ оператор $\int_{X^{2}} d x d y g^{(2)}(x, y) \partial_{y}^{\dagger} \partial_{x}$ является нулевым. Как следствие, на (2.10) не оказывает влияния тот факт, что мы не определили функцию $Q$ на $D$.

Для ограниченного линейного оператора $A$ на $\mathscr{H}$ определим его дифференциальное вторичное квантование $d \Gamma(A)$ как непрерывный линейный оператор на $\mathscr{F}_{\text {fin }}^{Q}(\mathscr{H})$, заданный формулами $d \Gamma(A) \Omega:=0$ и

$d \Gamma(A)\left\lceil\mathscr{H}^{\circledast n}:=\operatorname{Sym}_{n}(A \otimes \mathbf{1} \otimes \cdots \otimes \mathbf{1}+\mathbf{1} \otimes A \otimes \mathbf{1} \otimes \cdots \otimes \mathbf{1}+\cdots+\mathbf{1} \otimes \cdots \otimes \mathbf{1} \otimes A)\right.$

при каждом $n \in \mathbb{N}$. Для каждой ограниченной п. в. функции $h \in L^{\infty}(X, m)$ определим нейтральный оператор

$$
a^{0}(h):=\int_{X} d x h(x) \partial_{x}^{\dagger} \partial_{x} .
$$


Используя (2.8) и (2.9), получаем

$$
\begin{aligned}
\left(a^{0}(h) f^{(n)}\right)\left(x_{1}, \ldots, x_{n}\right) & =\left(\int_{X} d x h(x) \partial_{x}^{\dagger} f^{(n)}(x, \cdot)\right)\left(x_{1}, \ldots, x_{n}\right) \\
& =n \operatorname{Sym}_{n}\left(h\left(x_{1}\right) f^{(n)}\left(x_{1}, x_{2}, \ldots, x_{n}\right)\right) .
\end{aligned}
$$

Отсюда несложно вывести, что

$$
\left(a^{0}(h) f^{(n)}\right)\left(x_{1}, \ldots, x_{n}\right)=\left(h\left(x_{1}\right)+\cdots+h\left(x_{n}\right)\right) f^{(n)}\left(x_{1}, \ldots, x_{n}\right) .
$$

Как следствие, $a^{0}(h)=d \Gamma\left(M_{h}\right)$, где $M_{h}$ - оператор умножения на $h$.

\section{2. Энионный белый шум Леви и некоммутативные ортогональ-} ные многочлены. Для начала напомним конструкцию белого шума Леви над $X$ для энионной статистики (см. [18]). Пусть $\nu$ - вероятностная мера на $(\mathbb{R}, \mathscr{B}(\mathbb{R}))$. (На самом деле можно предполагать, что $\nu$ - конечная мера; в этом случае приводимые ниже результаты модифицируются очевидным образом.) Пусть $\mathscr{P}(\mathbb{R})$ - линейное пространство многочленов на $\mathbb{R}$. Мы предполагаем, что $\mathscr{P}(\mathbb{R})$ - плотное подмножество в $L^{2}(\mathbb{R}, \nu)$. Это предположение выполняется, к примеру, если имеет место условие (1.7).

Продолжим функцию $Q$, полагая

$$
Q\left(x_{1}, s_{1}, x_{2}, s_{2}\right):=Q\left(x_{1}, x_{2}\right), \quad\left(x_{1}, x_{2}\right) \in X^{(2)}, \quad\left(s_{1}, s_{2}\right) \in \mathbb{R}^{2} .
$$

Таким образом, значения функции $Q$ не зависят от $s_{1}$ и $s_{2}$. Аналогично $(2.3)$ вводится понятие $Q$-симметричной функции $f^{(n)}$, определенной на множестве

$$
\left\{\left(x_{1}, s_{1}, \ldots, x_{n}, s_{n}\right) \in(X \times \mathbb{R})^{n} \mid\left(x_{1}, \ldots, x_{n}\right) \in X^{(n)}\right\} .
$$

$\mathrm{K}$ примеру, если $n=2$, то $Q$-симметрия означает, что

$$
f^{(2)}\left(x_{1}, s_{1}, x_{2}, s_{2}\right)=Q\left(x_{1}, x_{2}\right) f^{(2)}\left(x_{2}, s_{2}, x_{1}, s_{1}\right) .
$$

Далее, полагая

$$
\mathscr{G}:=L^{2}(X \times \mathbb{R}, m \otimes \nu)=\mathscr{H} \otimes L^{2}(\mathbb{R}, \nu),
$$

рассмотрим соответствующее $Q$-симметричное фоковское пространство $\mathscr{F} Q(\mathscr{G})$, построение которого аналогично построению $\mathscr{F} Q(\mathscr{H})$. Пусть $\mathscr{F}_{\text {fin }}^{Q}(\mathscr{H} \otimes \mathscr{P}(\mathbb{R}))-$ линейное подпространство пространства $\mathscr{F}^{Q}(\mathscr{G})$, состоящее из конечных последовательностей

$$
F=\left(F^{(0)}, F^{(1)}, \ldots, F^{(n)}, 0,0, \ldots\right), \quad n \in \mathbb{N}_{0},
$$

в которых каждый элемент $F^{(k)}, k \neq 0$, имеет вид

$$
\begin{aligned}
F^{(k)} & \left(x_{1}, s_{1}, \ldots, x_{k}, s_{k}\right) \\
& =\operatorname{Sym}_{k}\left[\sum_{\left(i_{1}, i_{2}, \ldots, i_{k}\right) \in\{0,1, \ldots, N\}^{k}} f_{\left(i_{1}, i_{2}, \ldots, i_{k}\right)}\left(x_{1}, x_{2}, \ldots, x_{k}\right) s_{1}^{i_{1}} s_{2}^{i_{2}} \cdots s_{k}^{i_{k}}\right],
\end{aligned}
$$

где $f_{\left(i_{1}, i_{2}, \ldots, i_{k}\right)} \in \mathscr{H}^{\otimes k}$ и $N \in \mathbb{N}$. Ясно, что $\mathscr{F}_{\text {fin }}^{Q}(\mathscr{H} \otimes \mathscr{P}(\mathbb{R}))$ плотно в $\mathscr{F}^{Q}(\mathscr{G})$. 
Положим $1(s):=1$ и $\mathrm{id}(s):=s$ при $s \in \mathbb{R}$. Тогда $1, \mathrm{id} \in \mathscr{P}(\mathbb{R})$. Далее, пусть $C_{0}(X \rightarrow \mathbb{R})$ - пространство всех действительных непрерывных функций на $X$ с компактным носителем. Для каждой $f \in C_{0}(X \rightarrow \mathbb{R})$ определим оператор

$$
\langle\omega, f\rangle:=a^{+}(f \otimes 1)+a^{0}(f \otimes \mathrm{id})+a^{-}(f \otimes 1)
$$

на $\mathscr{F}^{Q}(\mathscr{G})$ с областью определения $\mathscr{F}_{\text {fin }}^{Q}(\mathscr{H} \otimes \mathscr{P}(\mathbb{R}))$. Ясно, что каждый оператор $\langle\omega, f\rangle$ отображает $\mathscr{F}_{\text {fin }}^{Q}(\mathscr{H} \otimes \mathscr{P}(\mathbb{R}))$ в себя. Если выполнено условие (1.7), то $F \in \mathscr{F}_{\text {fin }}^{Q}(\mathscr{H} \otimes \mathscr{P}(\mathbb{R}))$ является аналитическим вектором для каждого оператора $\langle\omega, f\rangle$, где $f \in C_{0}(X \rightarrow \mathbb{R})$. Это влечет, что операторы $\langle\omega, f\rangle$ являются существенно самосопряженными на $\mathscr{F}_{\text {fin }}^{Q}(\mathscr{H} \otimes \mathscr{P}(\mathbb{R}))($ см., например, [46; § X.2]).

ЗАмечАниЕ 2.2. Сохраним обозначение $\langle\omega, f\rangle$ для замыкания этого оператора в пространстве $\mathscr{F} Q(\mathscr{G})$. Тогда оператор $\langle\omega, f\rangle$ является самосопряженным. В бозонном случае, когда $q=1$, такие операторы также коммутируют в смысле коммутации их разложений единицы. Воспользовавшись, к примеру, проекционной спектральной теоремой [8], можно показать [24], что имеется унитарный изоморфизм между симметричным фоковским пространством $\mathscr{F} Q(\mathscr{G})$ и пространством $L^{2}\left(\mathscr{D}^{\prime}(X), \mu\right)$, где $\mu$ - белошумовая мера Леви с преобразованием Фурье (1.6). Под действием такого унитарного изоморфизма вакуумный вектор $\Omega$ переходит в постоянную функцию 1 , а каждый оператор $\langle\omega, f\rangle$ становится оператором умножения на случайную величину $\langle\omega, f\rangle$ из $L^{2}\left(\mathscr{D}^{\prime}(X), \mu\right)$. Иными словами, $\mu$ - это спектральная мера семейства коммутирующих самосопряженных операторов $(\langle\omega, f\rangle)_{f \in C_{0}(X \rightarrow \mathbb{R})}$. В частности, это означает, что операторы $(\langle\omega, f\rangle)_{f \in C_{0}(X \rightarrow \mathbb{R})}$ на симметричном фоковском пространстве $\mathscr{F}^{Q}(\mathscr{G})$ действительно можно рассматривать как белый шум Леви. Также отметим, что унитарный оператор, действующий между $\mathscr{F}^{Q}(\mathscr{G})$ и $L^{2}\left(\mathscr{D}^{\prime}(X), \mu\right)$, был впервые найден К. Ито [34] с использованием кратных стохастических интегралов.

ЗАмечАниЕ 2.3. Если мера $\nu$ сосредоточена в одной точке $\lambda \in \mathbb{R}$, то $\mathscr{G}=\mathscr{H}$ и каждый оператор $\langle\omega, f\rangle$ имеет следующий вид в $\mathscr{F} Q(\mathscr{H})$ :

$$
\langle\omega, f\rangle:=a^{+}(f)+a^{-}(f)+\lambda a^{0}(f) .
$$

Значение $\lambda=0$ соответствует энионному гауссовскому белому шуму, а $\lambda \neq 0$ энионному центрированному белому шуму. Положим

$$
\omega(x):=\partial_{x}^{\dagger}+\lambda \partial_{x}^{\dagger} \partial_{x}+\partial_{x}, \quad x \in X .
$$

Тогда, используя $(2.9),(2.13),(2.17)$ и $(2.18)$, находим

$$
\langle\omega, f\rangle=\int_{X} d x \omega(x) f(x), \quad f \in C_{0}(X \rightarrow \mathbb{R}),
$$

что оправдывает использование обозначения $\langle\omega, f\rangle$. Таким образом, $(\omega(x))_{x \in X}-$ энионный гауссовский или пуассоновский белый шум. Также отметим, что $\omega(x)$ можно неформально рассматривать как операторнозначное распределение.

Далее, пусть $C_{0}(X)$ - пространство непрерывных комплекснозначных функций на $X$ с компактным носителем. Для $f \in C_{0}(X)$ положим $\langle\omega, f\rangle:=\langle\omega, \operatorname{Re} f\rangle+$ $i\langle\omega, \operatorname{Im} f\rangle$. 
Пусть $\mathscr{P}$ - комплексная унитарная *-алгебра, порожденная $(\langle\omega, f\rangle)_{f \in C_{0}(X)}$, т. е. алгебра некоммутативных многочленов от переменных $\langle\omega, f\rangle$. В частности, элементы $\mathscr{P}$ - это линейные операторы, действующие на $\mathscr{F}_{\text {fin }}^{Q}(\mathscr{H} \otimes \mathscr{P}(\mathbb{R}))$ и такие, что для каждого $p \in \mathscr{P}$ оператор $p^{*}$ - это сопряженный оператор к $p$ на $\mathscr{F}^{Q}(\mathscr{G})$, суженный на $\mathscr{F}_{\text {fin }}^{Q}(\mathscr{H} \otimes \mathscr{P}(\mathbb{R}))$.

Определим вакуумное состояние на $\mathscr{P}$ формулой

$$
\tau(p):=(p \Omega, \Omega)_{\mathscr{F} Q(\mathscr{G})}, \quad p \in \mathscr{P} .
$$

Далее, введем на Я્Р скалярное произведение:

$$
\left(p_{1}, p_{2}\right)_{L^{2}(\tau)}:=\tau\left(p_{2}^{*} p_{1}\right)=\left(p_{1} \Omega, p_{2} \Omega\right)_{\mathscr{F} Q(\mathscr{G})}, \quad p_{1}, p_{2} \in \mathscr{P} .
$$

Положим

$$
\widetilde{\mathscr{P}}:=\left\{p \in \mathscr{P} \mid(p, p)_{L^{2}(\tau)}=0\right\}
$$

и определим некоммутативное $L^{2}$-пространство $L^{2}(\tau)$ как пополнение факторпространства $\mathscr{P} / \widetilde{\mathscr{P}}$ по отношению к норме, порождаемой скалярным произведением $(\cdot, \cdot)_{L^{2}(\tau)}$. Каждый элемент $p \in \mathscr{P}$ является представителем класса эквивалентности из факторпространства $\mathscr{P} / \widetilde{\mathscr{P}}$, поэтому $\mathscr{P}$ - плотное подпространство в $L^{2}(\tau)$. В [18] показано, что вакуумный вектор $\Omega$ является циклическим для семейства операторов $(\langle\omega, f\rangle)_{f \in C_{0}(X \rightarrow \mathbb{R})}$. Рассмотрим линейное отображение $I: \mathscr{P} \rightarrow \mathscr{F} Q(\mathscr{G})$, определяемое следующим образом:

$$
\text { Ip }:=p \Omega \quad \text { при } p \in \mathscr{P} .
$$

Тогда $I p_{1}=I p_{2}$, если $p_{1}, p_{2} \in \mathscr{P}$ таковы, что $p_{1}-p_{2} \in \widetilde{\mathscr{P}}$. При этом $I$ продолжается до унитарного оператора $I: L^{2}(\tau) \rightarrow \mathscr{F} Q(\mathscr{G})$.

Отметим, что при любых $p \in \mathscr{P}$ и $f \in C_{0}(X)$

$$
I(\langle\omega, f\rangle p)=\langle\omega, f\rangle(I p),
$$

т. е. под действием унитарного оператора $I$ оператор левого умножения $\langle\omega, f\rangle$ в $L^{2}(\tau)$ становится оператором $\langle\omega, f\rangle$ в $\mathscr{F}^{Q}(\mathscr{G})$.

Рассмотрим топологию на $C_{0}(X)$, порождающую следующую сходимость: $f_{n} \rightarrow f$ при $n \rightarrow \infty$ означает, что существует множество $\Delta \in \mathscr{B}_{0}(X)$ такое, что $\operatorname{supp}\left(f_{n}\right) \subset \Delta$ при всех $n \in \mathbb{N}$ и

$$
\sup _{x \in X}\left|f_{n}(x)-f(x)\right| \rightarrow 0, \quad n \rightarrow \infty .
$$

По линейности и непрерывности отображение

$$
C_{0}(X)^{n} \ni\left(f_{1}, \ldots, f_{n}\right) \mapsto\left\langle\omega^{\otimes n}, f_{1} \otimes \cdots \otimes f_{n}\right\rangle=\left\langle\omega, f_{1}\right\rangle \cdots\left\langle\omega, f_{n}\right\rangle \in \mathscr{P}
$$

продолжается до отображения

$$
C_{0}\left(X^{n}\right) \ni f^{(n)} \mapsto\left\langle\omega^{\otimes n}, f^{(n)}\right\rangle \in L^{2}(\tau),
$$

так что $\left\langle\omega^{\otimes n}, f^{(n)}\right\rangle$ может рассматриваться как линейный оператор, действующий на $\mathscr{F}_{\text {fin }}^{Q}(\mathscr{H} \otimes \mathscr{P}(\mathbb{R}))$. Мы будем рассматривать $\left\langle\omega^{\otimes n}, f^{(n)}\right\rangle$ как непрерывный 
моном порядка $n$. Суммы таких операторов и (комплексных) констант образуют множество $\mathscr{C} \mathscr{P}$ непрерывных многочленов (от $\omega)$. Ясно, что $\mathscr{P} \subset \mathscr{C} \mathscr{P}$.

Полностью аналогично (1.9) мы получаем ортогональное разложение

$$
L^{2}(\tau)=\bigoplus_{n=0}^{\infty} \mathscr{O} \mathscr{P}_{n}
$$

(обозначения очевидны). Для любого $f^{(n)} \in C_{0}\left(X^{n}\right)$ через $\left\langle P_{n}(\omega), f^{(n)}\right\rangle$ обозначим ортогональную проекцию $\left\langle\omega^{\otimes n}, f^{(n)}\right\rangle$ на $\mathscr{O} \mathscr{P}_{n}$, а через $\mathscr{O} \mathscr{C} \mathscr{P}$ будем обозначать класс конечных линейных сумм $\left\langle P_{n}(\omega), f^{(n)}\right\rangle$ и (комплексных) констант.

ЗАмечание 2.4. Отметим, что $\left\langle P_{1}(\omega), f\right\rangle=\langle\omega, f\rangle$.

ЗАмечАниЕ 2.5. В п. 1.2 множества $\mathscr{C} \mathscr{P}$ и $\mathscr{O} \mathscr{C} \mathscr{P}$ определялись с использованием функций $f^{(n)} \in \mathscr{D}(X)^{\otimes n}$, а в данном разделе $\mathscr{C} \mathscr{P}$ и $\mathscr{O} \mathscr{C} \mathscr{P}$ определяются через $f^{(n)} \in C_{0}\left(X^{n}\right)$. Причиной является то, что в некоммутативном случае нам нет нужды, чтобы $f^{(n)}$ была гладкой, в то время как в классическом случае $q=1$ теорема 1.2 остается верной для множеств $\mathscr{C} \mathscr{P}$ и $\mathscr{O} \mathscr{C} \mathscr{P}$, определенных в данном разделе.

\section{3. Основные результаты}

3.1. Меры $m_{\nu}^{(n)}$. Пусть $\left(p_{k}\right)_{k=0}^{\infty}-$ система приведенных ортогональных многочленов в $L^{2}(\mathbb{R}, \nu)$. (Если носитель $\nu$ конечен и состоит из $N$ точек, то мы полагаем $p_{k}:=0$ при $k \geqslant N$.) Соответственно, для системы $\left(p_{k}\right)_{k=0}^{\infty}$ имеет место рекуррентная формула

$$
s p_{k}(s)=p_{k+1}(s)+b_{k} p_{k}(s)+a_{k} p_{k-1}(s), \quad k \in \mathbb{N}_{0},
$$

где $p_{-1}(s):=0, a_{k}>0, b_{k} \in \mathbb{R}$. (Если носитель $\nu$ состоит из $N$ точек, то $a_{k}=0$ при $k \geqslant N$.)

Положим

$$
c_{k}:=a_{0} a_{1} \cdots a_{k-1}, \quad k \in \mathbb{N},
$$

где $a_{0}:=1$, а $a_{k}, k \in \mathbb{N}$, находятся из формулы (3.1). Это определение можно записать в эквивалентной форме:

$$
c_{k}=\int_{\mathbb{R}} p_{k-1}(s)^{2} \nu(d s), \quad k \in \mathbb{N} .
$$

Такое представление хорошо известно в теории ортогональных многочленов. Отметим, что $c_{1}=1$ и $c_{k}=0$ при $k \geqslant 2$ тогда и только тогда, когда мера $\nu$ сконцентрирована в одной точке.

Через $\Pi(n)$ обозначим множество всех (неупорядоченных) разбиений множества $\{1, \ldots, n\}$. Если $\theta=\left\{\theta_{1}, \ldots, \theta_{l}\right\} \in \Pi(n)$, то по определению полагаем $|\theta|:=l$. Далее, для каждого $\theta \in \Pi(n)$ через $X_{\theta}^{(n)}$ обозначим подмножество $X^{n}$, состоящее из всех $\left(x_{1}, \ldots, x_{n}\right) \in X^{n}$ таких, что при $1 \leqslant i<j \leqslant n$ равенство $x_{i}=x_{j}$ выполнено тогда и только тогда, когда $i$ и $j$ лежат в одном и том же 
элементе разбиения $\theta$. Отметим, что множества $X_{\theta}^{(n)}, \theta \in \Pi(n)$, образуют разбиение $X^{n}$. Также отметим, что, в силу определения $(2.1), X^{(n)}=X_{\theta}^{(n)}$ для минимального разбиения $\theta=\{\{1\},\{2\}, \ldots,\{n\}\}$.

Зафиксируем число $n \in \mathbb{N}$, перестановку $\pi \in \mathfrak{S}_{n}$ и разбиение $\theta=\left\{\theta_{1}, \ldots, \theta_{l}\right\} \in$ $\Pi(n)$, удовлетворяющее условию

$$
\max \theta_{1}<\max \theta_{2}<\cdots<\max \theta_{l} .
$$

Определим меру $m_{\nu, \theta}^{(n)}$ на $X_{\theta}^{(n)}$ как проекцию меры

$$
\left(c_{\left|\theta_{1}\right|} \cdots c_{\left|\theta_{l}\right|}\right) n !\left(\left|\theta_{1}\right| ! \cdots\left|\theta_{l}\right| !\right)^{-1} m^{\otimes l}
$$

на $X^{(l)}$ при отображении

$$
X^{(l)} \ni y=\left(y_{1}, \ldots, y_{l}\right) \mapsto\left(R_{\theta}^{1} y, \ldots, R_{\theta}^{n} y\right) \in X_{\theta}^{(n)},
$$

где $R_{\theta}^{i} y=y_{j}$ при $i \in \theta_{j}$. Здесь $\left|\theta_{i}\right|$ обозначает мощность множества $\theta_{i}$. Учитывая, что множества $X_{\theta}^{(n)}, \theta \in \Pi(n)$, образуют разбиение $X^{n}$, определим меpy $m_{\nu}^{(n)}$ на $X^{n}$ таким образом, чтобы сужение $m_{\nu}^{(n)}$ на каждое $X_{\theta}^{(n)}$ совпадало с $m_{\nu, \theta}^{(n)}$. Отметим, что сужение $m_{\nu}^{(n)}$ на $X^{(n)}$ совпадет с $n ! m^{\otimes n}$.

$\mathrm{K}$ примеру, при $n=2$ мы получаем

$$
\begin{aligned}
\int_{X^{2}} & f^{(2)}\left(x_{1}, x_{2}\right) m_{\nu}^{(2)}\left(d x_{1} \times d x_{2}\right) \\
& =\int_{\left\{x_{1} \neq x_{2}\right\}} f^{(2)}\left(x_{1}, x_{2}\right) d x_{1} d x_{2} 2+\int_{X} f^{(2)}(x, x) d x c_{2} \\
& =\int_{X^{2}} f^{(2)}\left(x_{1}, x_{2}\right) d x_{1} d x_{2} 2+\int_{X} f^{(2)}(x, x) d x c_{2} .
\end{aligned}
$$

3.2. Расширенное энионное фоковское пространство. В п. 2.1 (см., в частности, (2.3)) мы определили понятие $Q$-симметричной функции $f^{(n)}$ : $X^{(n)} \rightarrow \mathbb{C}$. Нашим следующим шагом будет перенесение данного определения на комплекснозначные функции, определенные на всем $X^{n}$.

Зафиксируем перестановку $\pi \in \mathfrak{S}_{n}$ и разбиение $\theta=\left\{\theta_{1}, \ldots, \theta_{l}\right\} \in \Pi(n)$, удовлетворяющее (3.4). Перестановка $\pi$ переводит разбиение $\theta$ в разбиение

$$
\left\{\pi \theta_{1}, \ldots, \pi \theta_{l}\right\} \in \Pi(n),
$$

которое мы называем новым разбиением $\beta=\left\{\beta_{1}, \ldots, \beta_{l}\right\}$, считая при этом, что элементы разбиения $\beta$ занумерованы так, что

$$
\max \beta_{1}<\max \beta_{2}<\cdots<\max \beta_{l} .
$$

Таким образом, перестановка $\pi \in \mathfrak{S}_{n}$ определяет перестановку $\widehat{\pi} \in \mathfrak{S}_{l}$ (зависящую от $\theta$ ), для которой

$$
\pi \theta_{i}=\beta_{\widehat{\pi}(i)}, \quad i=1, \ldots, l .
$$


Напомним, что комплекснозначная функция $Q_{\pi}\left(x_{1}, \ldots, x_{n}\right)$ на $X^{(n)}$ определена в (2.5). Чтобы распространить эту функцию на всё $X^{n}$, поступим следующим образом. Зафиксируем произвольное разбиение $\theta=\left\{\theta_{1}, \ldots, \theta_{l}\right\} \in \Pi(n)$, удовлетворяющее $(3.4)$, и зафиксируем произвольный набор $\left(x_{1}, \ldots, x_{n}\right) \in X_{\theta}^{(n)}$. Через $x_{\theta_{1}}, x_{\theta_{2}}, \ldots, x_{\theta_{l}}$ обозначим элементы $x_{i_{1}}, x_{i_{2}}, \ldots, x_{i_{l}}$, где $i_{1} \in \theta_{1}, i_{2} \in \theta_{2}$, $\ldots, i_{l} \in \theta_{l}$. Положим

$$
Q_{\pi}\left(x_{1}, \ldots, x_{n}\right):=\prod_{\substack{1 \leqslant i<j \leqslant l \\ \widehat{\pi}(i)>\widehat{\pi}(j)}} Q\left(x_{\theta_{i}}, x_{\theta_{j}}\right)
$$

где перестановка $\widehat{\pi} \in \mathfrak{S}_{l}$ определена выше. Отметим, что для разбиения

$$
\theta=\{\{1\},\{2\}, \ldots,\{n\}\}
$$

сужение функции $Q_{\pi}$ на множество $X_{\theta}^{(n)}=X^{(n)}$ в действительности совпадает с функцией $Q_{\pi}$, определенной в $(2.5)$.

Функция $f^{(n)}: X^{n} \rightarrow \mathbb{C}$ называется $Q$-симметричной, если для любой перестановки $\pi \in \mathfrak{S}_{n}$

$$
f^{(n)}\left(x_{1}, \ldots, x_{n}\right)=Q_{\pi}\left(x_{1}, \ldots, x_{n}\right) f^{(n)}\left(x_{\pi^{-1}(1)}, \ldots, x_{\pi^{-1}(n)}\right), \quad\left(x_{1}, \ldots, x_{n}\right) \in X^{n} .
$$

В частности, сужение такой функции на $X^{(n)}$ является $Q$-симметричным в соответствии с определением из п. 2.1, т. е. для него выполнено (2.3).

Далее, для функции $f^{(n)}: X^{n} \rightarrow \mathbb{C}$ определим

$$
\begin{aligned}
& \left(\operatorname{Sym}_{n} f^{(n)}\right)\left(x_{1}, \ldots, x_{n}\right)=\frac{1}{n !} \sum_{\pi \in \mathfrak{S}_{n}} Q_{\pi}\left(x_{1}, \ldots, x_{n}\right) \\
& \quad \times f^{(n)}\left(x_{\pi^{-1}(1)}, \ldots, x_{\pi^{-1}(n)}\right), \quad\left(x_{1}, \ldots, x_{n}\right) \in X^{n} .
\end{aligned}
$$

Ясно, что сужение функции $\operatorname{Sym}_{n} f^{(n)}$ на множество $X^{(n)}$ по-прежнему задается формулой (2.4).

Через $\mathbf{F}_{n}^{Q}(\mathscr{H}, \nu)$ обозначим подпространство комплексного $L^{2}$-пространства $L^{2}\left(X^{n}, m_{\nu}^{(n)}\right)$, состоящего из $\left(m_{\nu}^{(n)}\right.$-версий) $Q$-симметричных функций.

ПреДЛОЖениЕ 3.1. Для каждого $n \in \mathbb{N}$ оператор $\mathrm{Sym}_{n}$ является ортогоналъной проекиией пространства $L^{2}\left(X^{n}, m_{\nu}^{(n)}\right)$ на $\mathbf{F}_{n}^{Q}(\mathscr{H}, \nu)$.

Положим $\mathbf{F}_{0}^{Q}(\mathscr{H}, \nu)=\{c \Omega \mid c \in \mathbb{C}\}$, где $\Omega$ - вакуумный вектор. Определим расширенное энионное фоковское пространство следующим образом:

$$
\mathbf{F}^{Q}(\mathscr{H}, \nu):=\bigoplus_{n=0}^{\infty} \mathbf{F}_{n}^{Q}(\mathscr{H}, \nu) .
$$

Если мера $\nu$ сосредоточена в одной точке (и тогда $c_{1}=1$ и $c_{k}=0, k \geqslant 2$ ), то мы полагаем $\mathbf{F}^{Q}(\mathscr{H}, \nu)=\mathscr{F}^{Q}(\mathscr{H})$, т. е. $\mathbf{F}^{Q}(\mathscr{H}, \nu)-$ классическое энионное фоковское пространство. В противном случае $\mathscr{F} Q(\mathscr{H})$ является собственным 
подпространством пространства $\mathbf{F}^{\mathbf{Q}}(\mathscr{H}, \nu)$. Действительно, с помощью формулы (2.2) мы можем вложить $\mathscr{F}^{Q}(\mathscr{H})$ в $\mathbf{F}^{Q}(\mathscr{H}, \nu)$, идентифицируя произвольную функцию $f^{(n)} \in \mathscr{H}^{\circledast n}$ с функцией из $\mathbf{F}_{n}^{Q}(\mathscr{H}, \nu)$, которая совпадает с $f^{(n)}$ на $X^{(n)}$ и равна нулю вне его. Ясно, что в этом случае ортогональное дополнение к $\mathscr{F}^{Q}(\mathscr{H})$ в $\mathbf{F}^{Q}(\mathscr{H}, \nu)$ является ненулевым пространством.

Используя ортогональное разложение (2.22), мы построим унитарный изоморфизм между $L^{2}(\tau)$ и расширенным энионным фоковским пространством $\mathbf{F}^{Q}(\mathscr{H}, \nu)$.

Tеорема 3.2. Пусть $f^{(n)}, g^{(n)} \in C_{0}\left(X^{n}\right)$. Тогда

$$
\left(\left\langle P_{n}(\omega), f^{(n)}\right\rangle,\left\langle P_{n}(\omega), g^{(n)}\right\rangle\right)_{L^{2}(\tau)}=\left(\operatorname{Sym}_{n} f^{(n)}, \operatorname{Sym}_{n} g^{(n)}\right)_{\mathbf{F}_{n}^{Q}(\mathscr{H}, \nu)} .
$$

Так как $C_{0}\left(X^{n}\right)$ плотно в $L^{2}\left(X^{n}, m_{\nu}^{(n)}\right)$, то из теоремы 3.2 вытекает, что отображение

$$
C_{0}\left(X^{n}\right) \ni f^{(n)} \mapsto\left\langle P_{n}(\omega), f^{(n)}\right\rangle \in L^{2}(\tau)
$$

может быть продолжено до линейного непрерывного оператора

$$
L^{2}\left(X^{n}, m_{\nu}^{(n)}\right) \ni f^{(n)} \mapsto\left\langle P_{n}(\omega), f^{(n)}\right\rangle \in L^{2}(\tau) .
$$

Далее, по теореме 3.2 имеем, что при всех $f^{(n)} \in L^{2}\left(X^{n}, m_{\nu}^{(n)}\right)$

$$
\left\langle P_{n}(\omega), f^{(n)}\right\rangle=\left\langle P_{n}(\omega), \operatorname{Sym}_{n} f^{(n)}\right\rangle .
$$

Таким образом, непосредственным следствием теоремы 3.2 является следующее утверждение.

СлеДСТВиЕ 3.3. Имеет место унитарный изоморфизм:

$$
\mathbf{F}^{Q}(\mathscr{H}, \nu) \ni\left(f^{(n)}\right)_{n=0}^{\infty} \mapsto f^{(0)}+\sum_{n=1}^{\infty}\left\langle P_{n}(\omega), f^{(n)}\right\rangle \in L^{2}(\tau) .
$$

Пусть $U$ - обратный оператор к унитарному оператору (3.11). Тогда $U$ : $L^{2}(\tau) \rightarrow \mathbf{F}^{Q}(\mathscr{H}, \nu)$ - унитарный оператор (ср. с теоремой 1.4 , (ii) в бозонном случае, когда $q=1)$.

3.3. Энионный белый шум Леви как поле Якоби. С учетом результатов п. 2.2 и следствия 3.3 мы имеем следующую цепочку унитарных операторов:

$$
\mathbf{F}^{Q}(\mathscr{H}, \nu) \stackrel{U}{\longleftarrow} L^{2}(\tau) \stackrel{I}{\rightarrow} \mathscr{F}^{Q}(\mathscr{G}) .
$$

Мы также рассмотрим унитарный оператор

$$
\mathbf{U}: \mathbf{F}^{Q}(\mathscr{H}, \nu) \rightarrow \mathscr{F}^{Q}(\mathscr{G}), \quad \mathbf{U}:=I U^{-1} .
$$

Пусть $h \in C_{0}(X)$. Напомним, что согласно формуле (2.20) под действием $I^{-1}$ оператор $\langle\omega, h\rangle$, заданный на $\mathscr{F} Q(\mathscr{G})$, становится оператором умножения на $\langle\omega, h\rangle$ в $L^{2}(\tau)$. Положим

$$
\mathbf{J}(h):=\mathbf{U}^{-1}\langle\omega, h\rangle \mathbf{U} .
$$


Ясно, что операторы $\mathbf{J}(h)$ образуют поле Якоби в расширенном энионном фоковском пространстве $\mathbf{F}^{Q}(\mathscr{H}, \nu)$, т. е. каждый оператор $\mathbf{J}(h)$ имеет представление

$$
\mathbf{J}(h)=\mathbf{J}^{+}(h)+\mathbf{J}^{0}(f)+\mathbf{J}^{-}(h),
$$

где $\mathbf{J}^{+}(h)$ - оператор рождения, $\mathbf{J}^{0}(h)$ - нейтральный оператор, а $\mathbf{J}^{-}(h)$ - оператор уничтожения. В эквивалентном виде это записывается следующим образом:

$$
\begin{aligned}
\langle\omega, h\rangle\left\langle P_{n}(\omega), f^{(n)}\right\rangle=\langle & \left.P_{n+1}(\omega), \mathbf{J}^{+}(h) f^{(n)}\right\rangle+\left\langle P_{n}(\omega), \mathbf{J}^{0}(h) f^{(n)}\right\rangle \\
& +\left\langle P_{n-1}(\omega), \mathbf{J}^{-}(h) f^{(n)}\right\rangle .
\end{aligned}
$$

Нашей дальнейшей целью является точное вычисление операторов $\mathbf{J}^{\sharp}(h)$, $\sharp=+, 0,-$.

Определим линейное пространство $\mathscr{F}_{\text {fin }}\left(B_{0}(X)\right)$, состоящее из всех конечных векторов $\left(f^{(0)}, f^{(1)}, \ldots, f^{(n)}, 0,0, \ldots\right)$, где $f^{(0)} \in \mathbb{C}, f^{(i)} \in B_{0}\left(X^{i}\right), i \geqslant 1$. Ясно, что $\mathscr{F}_{\text {fin }}\left(B_{0}(X)\right)$ содержит вакуумный вектор $\Omega$.

Для каждого $h \in C_{0}(X)$ определим на $\mathscr{F}_{\text {fin }}\left(B_{0}(X)\right)$ нейтральный оператор $\mathscr{J}^{0}(h)$ и оператор уничтожения $\mathscr{J}_{1}^{-}(h)$ следующим образом. Для начала мы положим

$$
\mathscr{J}^{0}(h) \Omega=\mathscr{J}_{1}^{-}(h) \Omega:=0 .
$$

Далее, определим

$$
\left(\mathscr{J}^{0}(h) f^{(n)}\right)\left(x_{1}, \ldots, x_{n}\right):=\sum_{i=1}^{n} h\left(x_{i}\right) f^{(n)}\left(x_{1}, \ldots, x_{n}\right) R_{i}^{(n)}\left(x_{1}, \ldots, x_{n}\right) .
$$

Здесь для каждого $\theta=\left\{\theta_{1}, \ldots, \theta_{l}\right\} \in \Pi(n)$ сужение функции $R_{i}^{(n)}: X^{n} \rightarrow \mathbb{R}$ на множество $X_{\theta}^{(n)}$ задается как

$$
R_{i}^{(n)} \uparrow X_{\theta}^{(n)}:=\frac{b_{\gamma(i, \theta)-1}}{\gamma(i, \theta)} .
$$

В формуле $(3.16) \gamma(i, \theta):=\left|\theta_{u}\right|$, где $\theta_{u} \in \theta$ выбрано таким образом, что $i \in \theta_{u}$, $\mathrm{a}\left(b_{k}\right)_{k=0}^{\infty}-$ коэффициенты из (3.1). Окончательно, мы полагаем

$$
\begin{aligned}
& \left(\mathscr{J}_{1}^{-}(h) f^{(n)}\right)\left(x_{1}, \ldots, x_{n-1}\right):=\sum_{1 \leqslant i<j \leqslant n} h\left(x_{j-1}\right) \\
& \quad \times f^{(n)}(x_{1}, \ldots, x_{i-1}, \underbrace{x_{j-1}}_{\text {на } i \text {-м месте }}, x_{i}, x_{i+1}, \ldots, \underbrace{x_{j-1}}_{\text {на }}, \ldots, x_{n-1}) \\
& \quad \times S_{j-1}^{(n)}\left(x_{1}, \ldots, x_{n-1}\right),
\end{aligned}
$$

где для любого $\theta \in \Pi(n-1)$

$$
S_{j-1}^{(n)} \uparrow X_{\theta}^{(n-1)}:=\frac{2 a_{\gamma(j-1, \theta)}}{\gamma(j-1, \theta)(\gamma(j-1, \theta)+1)} .
$$

Здесь коэффициенты $\left(a_{k}\right)_{k=1}^{\infty}$ также взяты из (3.1). 
Определим

$$
\mathbf{F}_{\text {fin }}^{Q}\left(B_{0}(X)\right):=\operatorname{Sym} \mathscr{F}_{\text {fin }}\left(B_{0}(X)\right),
$$

где $\operatorname{Sym}$ - линейный оператор на $\mathscr{F}_{\text {fin }}\left(B_{0}(X)\right)$ такой, что $\operatorname{Sym} f^{(n)}:=\operatorname{Sym}_{n} f^{(n)}$ при $f^{(n)} \in B_{0}\left(X^{n}\right)$. Мы также обозначим $\mathbf{B}_{0}^{Q}\left(X^{n}\right):=\operatorname{Sym}_{n} B_{0}\left(X^{n}\right)$.

На $\mathbf{F}_{\text {fin }}^{Q}\left(B_{0}(X)\right)$ определим $Q$-симметричное тензорное произведение, полагая

$$
f^{(m)} \circledast g^{(n)}:=\operatorname{Sym}_{m+n}\left(f^{(m)} \otimes g^{(n)}\right)
$$

при $f^{(m)} \in \mathbf{B}_{0}^{Q}\left(X^{m}\right)$ и $g^{(m)} \in \mathbf{B}_{0}^{Q}\left(X^{n}\right)$ и продолжая далее по линейности. Здесь $f^{(m)} \otimes g^{(n)} \in B_{0}\left(X^{m+n}\right)$ задается в виде

$$
\left(f^{(m)} \otimes g^{(n)}\right)\left(x_{1}, \ldots, x_{m+n}\right)=f^{(m)}\left(x_{1}, \ldots, x_{m}\right) g^{(n)}\left(x_{m+1}, \ldots, x_{m+n}\right) .
$$

Ниже мы покажем, что тензорное произведение $\circledast$ является ассоциативным. Также стоит отметить, что сужение $f^{(m)} \circledast g^{(n)}$ на $X^{(m+m)}$ очевидным образом совпадает с произведением $f^{(m)} \circledast g^{(n)}$, определенным в п. 2.1.

ТеОрема 3.4. При любом $h \in C_{0}(X)$ оператор $\mathbf{J}(h)$ является линейным на $\mathbf{F}_{\text {fin }}^{Q}\left(B_{0}(X)\right)$ и имеет представление $(3.13)$. Более того, для каждого $F \in$ $\mathbf{F}_{\text {fin }}^{Q}\left(B_{0}(X)\right)$ имеют место тождества

$$
\mathbf{J}^{+}(h) F=h \circledast F, \quad \mathbf{J}^{0}(h) F=\operatorname{Sym}\left(\mathscr{J}^{0}(h) F\right)
$$

$u$

$$
\mathbf{J}^{-}(h)=\mathbf{J}_{1}^{-}(h)+\mathbf{J}_{2}^{-}(h)
$$

Здесъ

$$
\mathbf{J}_{1}^{-}(h) F=\operatorname{Sym}\left(\mathscr{J}_{1}^{-}(h) F\right)
$$

и длякаждого $f^{(n)} \in \mathbf{B}_{0}^{Q}\left(X^{n}\right)$

$$
\left(\mathbf{J}_{2}^{-}(h) f^{(n)}\right)\left(x_{1}, \ldots, x_{n-1}\right)=n \int_{X} d y h(y) f^{(n)}\left(y, x_{1}, \ldots, x_{n-1}\right) .
$$

3.4. Характеризация многочленов типа Мейкснера. Напомним, что операторы $\mathscr{J}^{0}(h)$ и $\mathscr{J}^{-}(h)$ были определены через коэффициенты рекуррентного соотношения (3.1) (т. е. через меру $\nu$ ), так что они не зависят от типа энионной статистики (не зависят от $Q$ ). Также напомним, что множество $\mathscr{O} \mathscr{C} \mathscr{P}$ ортогонализованных непрерывных многочленов было определено в п. 2.2.

Рассмотрим следующее условие.

$(\mathrm{C})$ Для каждого $h \in C_{0}(X \rightarrow \mathbb{R})$ линейные операторы $\mathbf{J}^{0}(h), \mathbf{J}_{1}^{-}(h)$ отображают множество $\mathscr{O} \mathscr{C} \mathscr{P}$ в себя.

Теорема 3.5. Предположим, что $q \neq-1$ или что $q=-1$ и носитель меры $\nu$ не является двухточечным множеством. В этом случае условие (C) выполнено тогда и только тогда, когда существуют константы $\lambda \in \mathbb{R}, \eta \geqslant 0$ такие, что коэфбициенты $a_{k}, b_{k}$ в рекуррентной формуле (3.1) имеют вид

$$
a_{k}=\eta k(k+1) \quad(k \in \mathbb{N}), \quad b_{k}=\lambda(k+1) \quad\left(k \in \mathbb{N}_{0}\right) .
$$


При этом если (3.22) выполнено, то для любых $h, f_{1}, \ldots, f_{n} \in C_{0}(X)$

$$
\begin{aligned}
& \mathbf{J}(h) f_{1} \circledast \cdots \circledast f_{n}=h \circledast f_{1} \circledast \cdots \circledast f_{n} \\
& \quad+\lambda \sum_{i=1}^{n} f_{1} \circledast \cdots \circledast f_{i-1} \circledast\left(h f_{i}\right) \circledast f_{i+1} \circledast \cdots \circledast f_{n} \\
& \quad+2 \eta \sum_{1 \leqslant i<j \leqslant n} f_{1} \circledast \cdots \circledast f_{i-1} \circledast f_{i+1} \circledast \cdots \circledast f_{j-1} \circledast\left(h f_{i} f_{j}\right) \circledast f_{j+1} \circledast \cdots \circledast f_{n} \\
& \quad+n \int_{X} d y h(y)\left(f_{1} \circledast \cdots \circledast f_{n}\right)(y, \cdot) .
\end{aligned}
$$

Таким образом, в классическом случае $q=1$ теорема 3.5 в точности дает класс Мейкснера бесконечномерных многочленов, который обсуждался в п. 1.2. Отметим, что определенный выше класс мер $\nu$ не зависит от $q$. При таком выборе $\nu$ мы называем $\left(\left\langle P_{n}(\omega), f^{(n)}\right\rangle\right)$ системой ортогональных (некоммутативных) многочленов типа Мейкснера для энионной статистики.

ЗАмечАниЕ 3.6. Если в фермионном случае $(q=-1)$ носитель меры $\nu$ является двухточечным множеством, нам пока не удается показать, что условие (C) не может быть выполнено, однако мы выдвигаем гипотезу, что это действительно так.

Следующий результат вполне очевиден.

ПреДЛОЖенИЕ 3.7. Для каждого $q \in \mathbb{C},|q|=1$, равенство $\mathscr{C} \mathscr{P}=\mathscr{O} \mathscr{C} \mathscr{P}$ имеет место в энионных гауссовском и пуассоновском случаях, т.е. когда формула (3.22) выполнена при $\lambda \in \mathbb{R} u \eta=0$.

Отметим, что из-за вида оператора $\mathbf{J}_{2}^{-}(h)($ см. $(3.21))$ равенство $\mathscr{C} \mathscr{P}=\mathscr{O} \mathscr{C} \mathscr{P}$ не имеет места, если $q \neq 1$ и мера $\nu$ не сосредоточена в одной точке. Тем не менее в классическом случае $(q=1)$ теорема 3.5 влечет теорему 1.2 .

3.5. Энионный белый шум типа Мейкснера. В данном пункте мы предположим, что (3.22) имеет место. Мы можем (по крайней мере неформально) определить

$$
\omega(x)=\left\langle\omega, \delta_{x}\right\rangle, \quad x \in X,
$$

так что при $h \in C_{0}(X)$

$$
\langle\omega, h\rangle=\int_{X} d x \omega(x) h(x) .
$$

Как следствие, $(\omega(x))_{x \in X}$ может рассматриваться как энионный белый шум типа Мейкснера.

При $x \in X$ определим оператор уничтожения $\partial_{x}$ как линейный оператор, действующий на $\mathbf{F}_{\text {fin }}^{Q}\left(B_{0}(X)\right)$ по формуле

$$
\left(\partial_{x} f^{(n)}\right)\left(x_{1}, \ldots, x_{n-1}\right):=n f^{(n)}\left(x, x_{1}, \ldots, x_{n-1}\right), \quad\left(x_{1}, \ldots, x_{n-1}\right) \in X^{n-1},
$$


где $f^{(n)} \in \mathbf{B}_{0}^{Q}\left(X^{n}\right)$. Тогда в силу (3.21) при $h \in C_{0}(X)$ мы можем интерпретировать оператор $\mathbf{J}_{2}^{-}(h)$ как интеграл

$$
\mathbf{J}_{2}^{-}(h)=\int_{X} d x h(x) \partial_{x} .
$$

Далее, введем “операторнозначное распределение" $X \ni x \mapsto \partial_{x}^{\dagger}$ следующим образом:

$$
\int_{X} d x h(x) \partial_{x}^{\dagger} f^{(n)}:=h \circledast f^{(n)},
$$

где $h \in C_{0}(X)$ и $f^{(n)} \in \mathbf{B}_{0}^{Q}\left(X^{n}\right)$. Иными словами, действие этого распределения можно понимать так: $\partial_{x}^{\dagger} f^{(n)}=\delta_{x} \circledast f^{(n)}$. Итак, мы можем записать

$$
\mathbf{J}^{+}(h)=\int_{X} d x h(x) \partial_{x}^{\dagger} .
$$

При $h \in C_{0}(X)$ нам потребуются операторы

$$
\int_{X} d x h(x) \partial_{x}^{\dagger} \partial_{x}, \quad \int_{X} d x h(x) \partial_{x}^{\dagger} \partial_{x} \partial_{x},
$$

действующие на $\mathbf{F}_{\text {fin }}^{Q}\left(B_{0}(X)\right)$. Принимая во внимание $(3.25)$ и $(3.27)$, при каждом $f^{(n)} \in \mathbf{B}_{0}^{Q}\left(X^{n}\right)$ получаем

$$
\begin{aligned}
\left(\int_{X} d x h(x) \partial_{x}^{\dagger} \partial_{x} f^{(n)}\right)\left(x_{1}, \ldots, x_{n}\right) & =n\left(\int_{X} d x h(x) \partial_{x}^{\dagger} f^{(n)}(x, \cdot)\right)\left(x_{1}, \ldots, x_{n}\right) \\
& =n \operatorname{Sym}_{n}\left(h\left(x_{1}\right) f^{(n)}\left(x_{1}, x_{2}, \ldots, x_{n}\right)\right)
\end{aligned}
$$

(ср. с (2.14)) и, далее,

$$
\begin{aligned}
\left(\int_{X}\right. & \left.d x h(x) \partial_{x}^{\dagger} \partial_{x} \partial_{x} f^{(n)}\right)\left(x_{1}, \ldots, x_{n-1}\right) \\
\quad= & n(n-1)\left(\int_{X} d x h(x) \partial_{x}^{\dagger} f^{(n)}(x, x, \cdot)\right)\left(x_{1}, \ldots, x_{n-1}\right) \\
\quad= & n(n-1) \operatorname{Sym}_{n-1}\left(h\left(x_{1}\right) f^{(n)}\left(x_{1}, x_{1}, x_{2}, x_{3}, \ldots, x_{n-1}\right)\right)
\end{aligned}
$$

Теорема 3.8. Предположим, что имеют место равенства (3.22). Тогда для любого $h \in C_{0}(X)$

$$
\begin{aligned}
\mathbf{J}^{0}(h) & =\int_{X} d x h(x) \lambda \partial_{x}^{\dagger} \partial_{x}, \\
\mathbf{J}_{1}^{-}(h) & =\int_{X} d x h(x) \eta \partial_{x}^{\dagger} \partial_{x} \partial_{x} .
\end{aligned}
$$

Таким образом,

$$
\mathbf{J}(h)=\int_{X} d x h(x)\left(\partial_{x}^{\dagger}+\lambda \partial_{x}^{\dagger} \partial_{x}+\eta \partial_{x}^{\dagger} \partial_{x} \partial_{x}+\partial_{x}\right) .
$$


Ввиду формулы (3.12) оператор $\mathbf{J}(h)$ является реализацией оператора $\langle\omega, h\rangle$ в расширенном энионном фоковском пространстве $\mathbf{F}^{Q}(\mathscr{H}, \nu)$. Таким образом (позволяя себе некоторую вольность в обозначениях), мы можем обозначить $\mathbf{J}(h)$ через $\langle\omega, h\rangle$. Используя (3.24) и (3.32), мы получаем следующее представление для энионного белого шума типа Мейкснера (реализованного в расширенном энионном фоковском пространстве $\left.\mathbf{F}^{Q}(\mathscr{H}, \nu)\right)$ :

$$
\omega(x)=\partial_{x}^{\dagger}+\lambda \partial_{x}^{\dagger} \partial_{x}+\eta \partial_{x}^{\dagger} \partial_{x} \partial_{x}+\partial_{x}
$$

ЗАмечАниЕ 3.9. Следует отметить, что для $q$-коммутационных соотношений, где $q$ является действительным числом из интервала $(-1,0)$ или из интервала $(0,1)$ (см. [3], [15], [19]), нет аналога $q$-процесса Леви, для которого имелось бы представление типа (3.32). Тем не менее, как показано в [22], существуют классические марковские процессы, для которых вероятности переходов являются мерами ортогональности $q$-мейкснеровских (ортогональных) многочленов на действительной прямой.

\section{4. Доказательства}

4.1. Доказательство предложения 3.1. Поскольку $\mathrm{Sym}_{1}=1$, достаточно доказать требуемое утверждение при $n \geqslant 2$. Следуя [18], мы вкратце напомним, как доказывается то, что оператор $\mathrm{Sym}_{n}$, задаваемый формулой (2.4), является ортогональной проекцией в пространстве $L^{2}\left(X^{n}, m^{\otimes n}\right)$. Для каждого $\pi \in \mathfrak{S}_{n}$ положим

$$
\left(\Psi_{\pi} f^{(n)}\right)\left(x_{1}, \ldots, x_{n}\right)=Q_{\pi}\left(x_{1}, \ldots, x_{n}\right) f^{(n)}\left(x_{\pi^{-1}(1)}, \ldots, x_{\pi^{-1}(n)}\right)
$$

при $\left(x_{1}, \ldots, x_{n}\right) \in X^{(n)}$. Таким образом, $\operatorname{Sym}_{n}=\frac{1}{n !} \sum_{\pi \in \mathfrak{S}_{n}} \Psi_{\pi}$ и $\Psi_{\pi}^{*}=\Psi_{\pi^{-1}}$, откуда следует, что $\mathrm{Sym}_{n}^{*}=\mathrm{Sym}_{n}$. Далее, для каждой перестановки $\varkappa \in \mathfrak{S}_{n}$

$$
\Psi_{\pi} \Psi_{\varkappa}=\Psi_{\varkappa \pi}
$$

Следовательно, на $X^{(n)}$

$$
\operatorname{Sym}_{n}^{2}=\frac{1}{(n !)^{2}} \sum_{\pi, \varkappa \in \mathfrak{S}_{n}} \Psi_{\pi} \Psi_{\varkappa}=\frac{1}{(n !)^{2}} \sum_{\pi \in \mathfrak{S}_{n}} \sum_{\varkappa \in \mathfrak{S}_{n}} \Psi_{\pi \varkappa}=\frac{1}{n !} \sum_{\varkappa \in \mathfrak{S}_{n}} \Psi_{\varkappa}=\operatorname{Sym}_{n} .
$$

Таким образом, $\mathrm{Sym}_{n}$ является ортогональной проекцией. Отметим, что формула (4.2) влечет при всех $\varkappa, \pi \in \mathfrak{S}_{n}$ равенство

$$
\begin{aligned}
& \left(\prod_{\substack{1 \leqslant i<j \leqslant n \\
\pi(i)>\pi(j)}} Q\left(x_{i}, x_{j}\right)\right)\left(\prod_{\substack{1 \leqslant k<l \leqslant n \\
\varkappa(k)>\varkappa(l)}} Q\left(x_{\pi^{-1}(k)}, x_{\pi^{-1}(l)}\right)\right) \\
& \quad=\prod_{\substack{1 \leqslant i<j \leqslant n \\
(\varkappa \pi)(i)>(\varkappa \pi)(j)}} Q\left(x_{i}, x_{j}\right), \quad\left(x_{1}, \ldots, x_{n}\right) \in X^{(n)} .
\end{aligned}
$$


Теперь рассмотрим линейный ограниченный оператор $\mathrm{Sym}_{n}$ в пространстве $L^{2}\left(X^{n}, m_{\nu}^{(n)}\right)$. Представим оператор $\operatorname{Sym}_{n}$ в виде

$$
\operatorname{Sym}_{n}=\frac{1}{n !} \sum_{\pi \in \mathfrak{S}_{n}} \Psi_{\pi}
$$

где оператор $\Psi_{\pi} f^{(n)}$ определен на всем $X^{n}$ формулой (4.1), в правой части которой функция $Q_{\pi}\left(x_{1}, \ldots, x_{n}\right)$ на $X^{n}$ определена в п. 3.2.

Зафиксируем перестановку $\pi \in \mathfrak{S}_{n}$ и разбиение $\theta=\left\{\theta_{1}, \ldots, \theta_{l}\right\} \in \Pi(n)$, удовлетворяющее условию (3.4). Предположим, что (3.5), (3.6) выполнены. Далее, пусть $\varkappa \in \mathfrak{S}_{n}$ и $\zeta=\left\{\zeta_{1}, \ldots, \zeta_{l}\right\} \in \Pi(n)$ таковы, что

$$
\max \zeta_{1}<\max \zeta_{2}<\cdots<\max \zeta_{l}
$$

и

$$
\varkappa \beta_{i}=\zeta_{\widehat{\varkappa}(i)}, \quad i=1, \ldots, l,
$$

где $\widehat{\varkappa} \in \mathfrak{S}_{l}$.

Тогда для произвольных функции $f^{(n)}: X^{n} \rightarrow \mathbb{C}$ и набора $\left(x_{1}, \ldots, x_{n}\right) \in X^{n}$ имеем

$$
\left(\Psi_{\pi} \Psi_{\varkappa} f^{(n)}\right)\left(x_{1}, \ldots, x_{n}\right)=\left(\prod_{\substack{1 \leqslant i<j \leqslant l \\ \widehat{\pi}(i)>\pi(j)}} Q\left(x_{\theta_{i}}, x_{\theta_{j}}\right)\right)\left(\Psi_{\varkappa} f^{(n)}\right)\left(x_{\pi^{-1}(1)}, \ldots, x_{\pi^{-1}(n)}\right) .
$$

Положим $y_{i}=x_{\pi^{-1}(i)}$ (это эквивалентно тому, что $y_{\pi(i)}=x_{i}$ при $\left.i=1, \ldots, n\right)$. Таким образом, $y_{\pi(i)}=y_{\pi(j)}$ тогда и только тогда, когда $i$ и $j$ лежат в одном и том же элементе разбиения $\theta$. Эквивалентно, $y_{i}=y_{j}$ тогда и только тогда, когда $i$ и $j$ принадлежат одному и тому же элементу разбиения $\beta$. Следовательно,

$$
\left(\Psi_{\varkappa} f^{(n)}\right)\left(y_{1}, \ldots, y_{n}\right)=\left(\prod_{\substack{1 \leqslant u<v \leqslant l \\ \varkappa(u)>\varkappa(v)}} Q\left(y_{\beta_{u}}, y_{\beta_{v}}\right)\right) f^{(n)}\left(y_{\varkappa^{-1}(1)}, \ldots, y_{\varkappa^{-1}(n)}\right),
$$

откуда

$$
\begin{aligned}
\left(\Psi_{\varkappa} f^{(n)}\right)\left(x_{\pi^{-1}(1)}, \ldots, x_{\pi^{-1}(n)}\right) & \left(\prod_{\substack{1 \leqslant u<v \leqslant l \\
\hat{\varkappa}(u)>\hat{\varkappa}(v)}} Q\left(x_{\pi^{-1} \beta_{u}}, x_{\pi^{-1} \beta_{v}}\right)\right) f^{(n)}\left(x_{\pi^{-1} \varkappa^{-1}(1)}, \ldots, x_{\pi^{-1} \varkappa^{-1}(n)}\right) \\
& =\left(\prod_{\substack{1 \leqslant u<v \leqslant l \\
\hat{\varkappa}(u)>\hat{\varkappa}(v)}} Q\left(x_{\theta_{\widehat{\pi}-1}(u)}, x_{\theta_{\widehat{\pi}-1}(v)}\right)\right) f^{(n)}\left(x_{(\varkappa \pi)^{-1}(1)}, \ldots, x_{(\varkappa \pi)^{-1}(n)}\right),
\end{aligned}
$$

где мы учли, что при каждом $u=1, \ldots, l$

$$
\pi^{-1} \beta_{u}=\theta_{\widehat{\pi}^{-1}(u)}
$$


Далее, из (4.4) имеем, что

$$
\begin{aligned}
& \left(\prod_{\substack{1 \leqslant i<j \leqslant l \\
\hat{\pi}(i)>\widehat{\pi}(j)}} Q\left(x_{\theta_{i}}, x_{\theta_{j}}\right)\right)\left(\prod_{\substack{1 \leqslant u<v \leqslant l \\
\hat{\varkappa}(u)>\hat{\varkappa}(v)}} Q\left(x_{\theta_{\widehat{\pi}-1}(u)}, x_{\theta_{\hat{\pi}-1}(v)}\right)\right) \\
& =\left(\prod_{\substack{1 \leqslant i<j \leqslant l \\
\hat{\varkappa}(i)>\hat{\varkappa}(j)}} Q\left(x_{\theta_{i}}, x_{\theta_{j}}\right)\right) .
\end{aligned}
$$

Здесь $\widehat{\varkappa \pi}$ - перестановка из $\mathfrak{S}_{l}$, индуцированная перестановкой $\varkappa \pi \in \mathfrak{S}_{n}$ и разбиением $\theta$. При выводе (4.9) мы воспользовались тем, что $\widehat{\varkappa \pi}=\widehat{\varkappa} \widehat{\pi}$. Теперь, подставляя (4.8) в (4.7) и применяя (4.9), находим, что

$$
\Psi_{\pi} \Psi_{\varkappa}=\Psi_{\varkappa \pi}
$$

откуда, аналогично (4.3), получаем равенство $\mathrm{Sym}_{n}^{2}=\mathrm{Sym}_{n}$.

Далее, отметим, что мера $m_{\nu}^{(n)}$ инвариантна относительно преобразования

$$
X^{n} \ni\left(x_{1}, \ldots, x_{n}\right) \mapsto\left(x_{\pi^{-1}(1)}, \ldots, x_{\pi^{-1}(n)}\right) \in X^{n} .
$$

Более того, легко проверить, что равенство

$$
\overline{Q_{\pi^{-1}}\left(x_{\pi^{-1}(1)}, \ldots, x_{\pi^{-1}(n)}\right)}=Q_{\pi}\left(x_{1}, \ldots, x_{n}\right)
$$

имеет место при всех $\left(x_{1}, \ldots, x_{n}\right) \in X^{n}$. Отсюда следует, что $\Psi_{\pi}^{*}=\Psi_{\pi^{-1}}$ при всех $\pi \in \mathfrak{S}_{n}$, и, значит, $\operatorname{Sym}_{n}^{*}=\operatorname{Sym}_{n}$.

Таким образом, $\operatorname{Sym}_{n}$ является ортогональной проекцией в $L^{2}\left(X^{n}, m_{\nu}^{(n)}\right)$. Аналогично предложению 2.5 из [18] легко вытекает, что $\mathbf{F}_{n}^{Q}(\mathscr{H}, \nu)$ является образом $\mathrm{Sym}_{n}$. Это завершает доказательство предложения 3.1.

Напомним, что тензорное произведение $\circledast$ задано на $\mathbf{F}_{\text {fin }}^{Q}\left(B_{0}(X)\right)$ формулой (3.19). Используя (4.5), (4.10), легко показать, что

$$
\begin{aligned}
\left(\operatorname{Sym}_{m} f^{(m)}\right) \circledast\left(\operatorname{Sym}_{n} g^{(n)}\right) & =\operatorname{Sym}_{m+n}\left(\left(\operatorname{Sym}_{m} f^{(m)}\right) \otimes\left(\operatorname{Sym}_{n} g^{(n)}\right)\right) \\
& =\operatorname{Sym}_{m+n}\left(f^{(m)} \otimes g^{(n)}\right)
\end{aligned}
$$

при всех $f^{(m)} \in B_{0}\left(X^{m}\right)$ и $g^{(n)} \in B_{0}\left(X^{n}\right)$. Это показывает, что операция взятия тензорного произведения $\circledast$ ассоциативна на $\mathbf{F}_{\text {fin }}^{Q}\left(B_{0}(X)\right)$.

4.2. Доказательство теоремы 3.2. Наша следующая цель - установить явный вид подпространства $I \mathscr{O} \mathscr{P}_{n}$ пространства $\mathscr{F} Q(\mathscr{G})$, где унитарный опеpaтор $I: L^{2}(\tau) \rightarrow \mathscr{F}^{Q}(\mathscr{G})$ определен в п. 2.2.

Через $\mathbb{N}_{0, \text { fin }}^{\infty}$ обозначим множество всех бесконечных последовательностей $\alpha=\left(\alpha_{0}, \alpha_{1}, \alpha_{2}, \ldots\right) \in \mathbb{N}_{0}^{\infty}$, у которых только конечное число элементов $\alpha_{j}$ отличны от нуля. Положим $|\alpha|:=\alpha_{0}+\alpha_{1}+\alpha_{2}+\cdots$. Для каждого $\alpha \in \mathbb{N}_{0}^{\infty}$, fin, $|\alpha| \geqslant 1$, через $\mathscr{F}_{\alpha}$ обозначим подпространство фоковского пространства $\mathscr{F} Q(\mathscr{G})$, состоящее из всех элементов вида

$$
\begin{aligned}
& \operatorname{Sym}_{|\alpha|}\left(f^{(|\alpha|)}\left(x_{1}, \ldots, x_{|\alpha|}\right) p_{0}\left(s_{1}\right) \cdots p_{0}\left(s_{\alpha_{0}}\right)\right. \\
& \left.\quad \times p_{1}\left(s_{\alpha_{0}+1}\right) \cdots p_{1}\left(s_{\alpha_{0}+\alpha_{1}}\right) p_{2}\left(s_{\alpha_{0}+\alpha_{1}+1}\right) \cdots\right),
\end{aligned}
$$


где $f^{(|\alpha|)} \in \mathscr{H}^{\otimes|\alpha|}$. Для $\alpha \in \mathbb{N}_{0, \text { fin }}^{\infty},|\alpha|=0$, положим $\mathscr{F}_{\alpha}:=\{c \Omega \mid c \in \mathbb{C}\}$. Следующий результат из [18; $\S 7]$ является аналогом разложения Нуаларта-Схоутенса $L^{2}$-пространства классических процессов Леви [45] (см. также [48]).

ПРЕДЛОЖЕНИЕ 4.1. Имеет место разложение

$$
\mathscr{F}^{Q}(\mathscr{G})=\bigoplus_{\alpha \in \mathbb{N}_{0, \text { fin }}^{\infty}} \mathscr{F}_{\alpha} .
$$

Для каждого $n \in \mathbb{N}_{0}$ положим

$$
\mathbb{F}_{n}:=\bigoplus_{\substack{\alpha \in \mathbb{N}_{0, \text { fin }}^{\infty} \\ \alpha_{0}+2 \alpha_{1}+3 \alpha_{2}+\cdots=n}} \mathscr{F}_{\alpha} .
$$

Отметим, что по (4.11)

$$
\mathscr{F}^{Q}(\mathscr{G})=\bigoplus_{n=0}^{\infty} \mathbb{F}_{n} .
$$

ПреДЛОЖЕНИЕ 4.2. Для каждого $n \in \mathbb{Z}_{+}$

$$
I \mathscr{O} \mathscr{P}_{n}=\mathbb{F}_{n} .
$$

ДокАЗАтЕЛьство. Достаточно показать, что для каждого $n \in \mathbb{N}$

$$
I \mathscr{M} \mathscr{P}_{n}=\bigoplus_{\substack{\alpha \in \mathbb{N}_{0, \text { fin }}^{\infty} \\ \alpha_{0}+2 \alpha_{1}+3 \alpha_{2}+\cdots \leqslant n}} \mathscr{F}_{\alpha}=: \mathbb{M}_{n} .
$$

Лемма 4.3. Пространство $\mathbb{M}_{n}$ состоит из всех конечных сумм элементов вида

$$
\operatorname{Sym}_{k}\left(f^{(k)}\left(x_{1}, \ldots, x_{k}\right) s_{1}^{i_{1}} s_{2}^{i_{2}} \cdots s_{k}^{i_{k}}\right),
$$

где $f^{(k)} \in \mathscr{H}^{\otimes k} u i_{1}+i_{2}+\cdots+i_{k}+k \leqslant n$.

ДокАЗАТЕльство. Для каждого $\pi \in \mathfrak{S}_{k}$ определим унитарный оператор $\Psi_{\pi}$ на $\left(\mathscr{H} \otimes L^{2}(\mathbb{R}, \nu)\right)^{\otimes k}$ следующим образом:

$$
\begin{aligned}
& \left(\Psi_{\pi} g^{(k)}\right)\left(x_{1}, s_{1}, \ldots, x_{k}, s_{k}\right)=Q_{\pi}\left(x_{1}, \ldots, x_{k}\right) \\
& \quad \times g^{(k)}\left(x_{\pi^{-1}(1)}, s_{\pi^{-1}(1)}, \ldots, x_{\pi^{-1}(k)}, s_{\pi^{-1}(k)}\right) .
\end{aligned}
$$

Здесь функция $Q_{\pi}$ задана формулой $(2.5)$. В силу [18] операторы $\Psi_{\pi}$ образуют унитарное представление симметрической группы $\mathfrak{S}_{k}$, при этом $\mathrm{Sym}_{k}=$ $\operatorname{Sym}_{k} \Psi_{\pi}$ для каждого $\pi \in \mathfrak{S}_{k}$. Таким образом,

$$
\begin{aligned}
\operatorname{Sym}_{k} & \left(f^{(k)}\left(x_{1}, \ldots, x_{k}\right) r^{(k)}\left(s_{1}, \ldots, s_{k}\right)\right) \\
& =\operatorname{Sym}_{k}\left(u^{(k)}\left(x_{1}, \ldots, x_{k}\right) r^{(k)}\left(s_{\pi^{-1}(1)}, \ldots, s_{\pi^{-1}(k)}\right)\right)
\end{aligned}
$$

для любой перестановки $\pi \in \mathfrak{S}_{k}, f^{(k)} \in \mathscr{H}^{\otimes k}$ и любого многочлена $r^{(k)}\left(s_{1}, \ldots, s_{k}\right)$ от переменных $s_{1}, \ldots, s_{k}$, где

$$
u^{(k)}\left(x_{1}, \ldots, x_{k}\right)=Q_{\pi}\left(x_{1}, \ldots, x_{k}\right) f^{(k)}\left(x_{\pi^{-1}(1)}, \ldots, x_{\pi^{-1}(k)}\right)
$$

(в частности, $u^{(k)} \in \mathscr{H}^{\otimes k}$ ). 
Теперь утверждение леммы следует из очевидных представлений

$$
p_{l}(s)=\sum_{i=0}^{l} \alpha_{i l} s^{i}, \quad s^{l}=\sum_{i=0}^{l} \beta_{i l} p_{i}(s) .
$$

Вернемся к доказательству разложения (4.12). Пусть $\mathscr{F}_{\text {fin }}(\mathscr{H} \otimes \mathscr{P}(\mathbb{R}))$ линейное подпространство полного фоковского пространства над $\mathscr{H} \otimes L^{2}(\mathbb{R}, \nu)$, состоящее из конечных сумм элементов $c \Omega(c \in \mathbb{C})$ и элементов вида

$$
f^{(k)}\left(x_{1}, \ldots, x_{k}\right) s_{1}^{i_{1}} s_{2}^{i_{2}} \cdots s_{k}^{i_{k}}
$$

где $f^{(k)} \in \mathscr{H}^{\otimes k}, i_{1}, i_{2}, \ldots, i_{k} \in \mathbb{Z}_{+}, k \in \mathbb{N}$. Положим

$$
\mathrm{Sym}:=\mathbf{1} \oplus \mathrm{Sym}_{1} \oplus \mathrm{Sym}_{2} \oplus \mathrm{Sym}_{3} \oplus \cdots .
$$

Этот оператор проецирует $\mathscr{F}_{\text {fin }}(\mathscr{H} \otimes \mathscr{P}(\mathbb{R}))$ на $\mathscr{F}_{\text {fin }}^{Q}(\mathscr{H} \otimes \mathscr{P}(\mathbb{R}))$. Для любых $h \in C_{0}(X)$ и $F \in \mathscr{F}_{\text {fin }}(\mathscr{H} \otimes \mathscr{P}(\mathbb{R}))$ имеем

$$
\begin{aligned}
& a^{+}(h \otimes 1) \operatorname{Sym} F=\operatorname{Sym}\left(J^{+}(h \otimes 1) F\right), \\
& a^{-}(h \otimes 1) \operatorname{Sym} F=\operatorname{Sym}\left(J^{-}(h \otimes 1) F\right)
\end{aligned}
$$

и

$$
a^{0}(h \otimes \mathrm{id}) \operatorname{Sym} F=\operatorname{Sym}\left(J^{0}(h \otimes \mathrm{id}) F\right) .
$$

Здесь для каждого $F$ вида (4.14)

$$
\begin{aligned}
& \left(J^{+}(h \otimes 1) F\right)\left(x_{1}, s_{1}, \ldots, x_{k+1}, s_{k+1}\right)=h\left(x_{1}\right) 1\left(s_{1}\right) f^{(k)}\left(x_{2}, \ldots, x_{k+1}\right) s_{2}^{i_{1}} s_{3}^{i_{2}} \cdots s_{k+1}^{i_{k}}, \\
& \left(J^{0}(h \otimes \mathrm{id}) F\right)\left(x_{1}, s_{1}, \ldots, x_{k}, s_{k}\right)=\left(h\left(x_{1}\right) s_{1}+\cdots+h\left(x_{k}\right) s_{k}\right) \\
& \quad \times f^{(k)}\left(x_{1}, \ldots, x_{k}\right) s_{1}^{i_{1}} s_{2}^{i_{2}} \cdots s_{k}^{i_{k}} \\
& \quad\left(J^{-}(h \otimes 1) F\right)\left(x_{1}, s_{1}, \ldots, x_{k-1}, s_{k-1}\right) \\
& \quad \sum_{j=1}^{k} \int_{X} d y \int_{\mathbb{R}} \nu(d t) h(y) Q\left(y, x_{1}\right) \cdots Q\left(y, x_{j-1}\right) \\
& \quad \times f^{(k)}\left(x_{1}, \ldots, x_{j-1}, y, x_{j}, \ldots, x_{k-1}\right) s_{1}^{i_{1}} \cdots s_{j-1}^{i_{j-1}} t^{i_{j}} s_{j}^{i_{j+1}} \cdots b s_{k-1}^{i_{j_{k}}} .
\end{aligned}
$$

Из леммы 4.3 и соотношений (4.16)-(??) по индукции следует, что

$$
\left\langle\omega, h_{1}\right\rangle \cdots\left\langle\omega, h_{n}\right\rangle \Omega \subset \mathbb{M}_{n}
$$

при всех $h_{1}, \ldots, h_{n} \in C_{0}(X), n \in \mathbb{N}$. Так как $\mathbb{M}_{n}$ - замкнутое подпространство пространства $\mathscr{F}^{Q}(\mathscr{G})$, то мы имеем включение $I \mathscr{M} \mathscr{P}_{n} \subset \mathbb{M}_{n}$. С другой стороны, из доказательства предложения 6.7 работы [18] непосредственно следует, что каждый элемент $\mathbb{M}_{n}$, имеющий вид $(4.13)$, лежит в $I \mathscr{M} \mathscr{P}_{n}$. Это доказывает обратное включение $\mathbb{M}_{n} \subset I \mathscr{M} \mathscr{P}_{n}$. Предложение 4.2 доказано. 
Отметим, что для каждого $h \in C_{0}(X)$

$$
a^{0}(h \otimes \mathrm{id})=d \Gamma\left(M_{h \otimes \mathrm{id}}\right)=d \Gamma\left(M_{h} \otimes M_{\mathrm{id}}\right),
$$

где $M_{h}$ - оператор умножения на функцию $h(x)$ из $\mathscr{H}$, а $M_{\text {id }}-$ (суженный на $\mathscr{P}(\mathbb{R})$ ) оператор умножения на моном $\operatorname{id}(s)=s$ из $L^{2}(\mathbb{R}, \nu)$. Стоит упомянуть, что оператор $M_{\text {id }}$ неограничен в $L^{2}(\mathbb{R}, \nu)$, если носитель меры $\nu$ неограничен и оператор вторичного квантования имеет область определения $\mathscr{F}_{\text {fin }}^{Q}(\mathscr{H} \otimes \mathscr{P}(\mathbb{R}))$. С учетом рекуррентной формулы (3.1) мы получаем представление

$$
M_{\text {id }}=A^{+}+A^{0}+A^{-},
$$

где $A^{+}, A^{0}, A^{-}$- линейные операторы на $\mathscr{P}(\mathbb{R})$, определяемые формулами

$$
A^{+} p_{k}:=p_{k+1}, \quad A^{0} p_{k}:=b_{k} p_{k}, \quad A^{-} p_{k}:=a_{k} p_{k-1}
$$

По (4.19) и (4.20)

$$
a^{0}(h \otimes \mathrm{id})=d \Gamma\left(M_{h} \otimes A^{+}\right)+d \Gamma\left(M_{h} \otimes A^{0}\right)+d \Gamma\left(M_{h} \otimes A^{-}\right) .
$$

Далее, из (2.16) и (4.21) вытекает, что при всех $h \in C_{0}(X)$

$$
\langle\omega, h\rangle=\mathscr{A}^{+}(h)+\mathscr{A}^{0}(h)+\mathscr{A}^{-}(h),
$$

где

$$
\begin{aligned}
& \mathscr{A}^{+}(h):=a^{+}(h \otimes 1)+d \Gamma\left(M_{h} \otimes A^{+}\right), \\
& \mathscr{A}^{0}(h):=d \Gamma\left(M_{h} \otimes A^{0}\right), \\
& \mathscr{A}^{-}(h):=a^{-}(h \otimes 1)+d \Gamma\left(M_{h} \otimes A^{-}\right) .
\end{aligned}
$$

ПРЕДЛОЖЕНИЕ 4.4. При каждом $h \in C_{0}(X)$ имеем:

$$
\mathscr{A}^{+}(h): \mathbb{F}_{n} \rightarrow \mathbb{F}_{n+1}, \quad \mathscr{A}^{0}(h): \mathbb{F}_{n} \rightarrow \mathbb{F}_{n}, \quad \mathscr{A}^{-}(h): \mathbb{F}_{n} \rightarrow \mathbb{F}_{n-1} .
$$

ДоказАтельство. Пусть $\sharp=+, 0,-$. Для любого $h \in C_{0}(X)$ определим на $\mathscr{F}_{\text {fin }}(\mathscr{H} \otimes \mathscr{P}(\mathbb{R}))$ оператор $N\left(M_{h} \otimes A^{\sharp}\right)$ следующим образом: $N\left(M_{h} \otimes A^{\sharp}\right) \Omega:=0$; далее, для каждого $n \in \mathbb{N}$ положим

$$
\begin{gathered}
N\left(M_{h} \otimes A^{\sharp}\right) \uparrow\left(\mathscr{F}_{\text {fin }}(\mathscr{H} \otimes \mathscr{P}(\mathbb{R})) \cap \mathscr{G}^{\otimes n}\right):=\left(M_{h} \otimes A^{\sharp}\right) \otimes \mathbf{1} \otimes \cdots \otimes \mathbf{1} \\
+\mathbf{1} \otimes\left(M_{h} \otimes A^{\sharp}\right) \otimes \mathbf{1} \otimes \cdots \otimes \mathbf{1}+\cdots+\mathbf{1} \otimes \cdots \otimes \mathbf{1} \otimes\left(M_{h} \otimes A^{\sharp}\right) .
\end{gathered}
$$

Лемма 4.5. Пусть $\sharp=+, 0,-$. Тогда для каждьх $h \in C_{0}(X \rightarrow \mathbb{R})$ и $F \in$ $\mathscr{F}_{\text {fin }}(\mathscr{H} \otimes \mathscr{P}(\mathbb{R}))$ имеем

$$
d \Gamma\left(M_{h} \otimes A^{\sharp}\right) \operatorname{Sym} F=\operatorname{Sym}\left(N\left(M_{h} \otimes A^{\sharp}\right) F\right) .
$$


ДокАЗАТЕльство. Зафиксируем произвольно $F \in \mathscr{F}_{\text {fin }}(\mathscr{H} \otimes \mathscr{P}(\mathbb{R}))$ вида

$$
F\left(x_{1}, s_{1}, \ldots, x_{n}, s_{n}\right)=f^{(n)}\left(x_{1}, \ldots, x_{n}\right) p_{i_{1}}\left(s_{1}\right) \cdots p_{i_{n}}\left(s_{n}\right) .
$$

Используя (2.4), находим, что

$$
\begin{aligned}
& \left(\operatorname{Sym}_{n} F\right)\left(x_{1}, s_{1}, \ldots, x_{k}, s_{k}\right)=\frac{1}{n !} \sum_{\pi \in \mathfrak{S}_{n}} Q_{\pi}\left(x_{1}, \ldots, x_{n}\right) \\
& \times f^{(n)}\left(x_{\pi^{-1}(1)}, \ldots, x_{\pi^{-1}(n)}\right) p_{i_{1}}\left(s_{\pi(1)}\right) \cdots p_{i_{n}}\left(s_{\pi(n)}\right) .
\end{aligned}
$$

Заметим, что

$$
d \Gamma\left(M_{h} \otimes A^{+}\right)=\operatorname{Sym}\left(N\left(M_{h} \otimes A^{+}\right)\right) .
$$

В силу (4.24)

$$
\begin{aligned}
\left(N\left(M_{h} \otimes A^{+}\right) \operatorname{Sym}_{n} F\right)\left(x_{1}, s_{1}, \ldots, x_{n}, s_{n}\right) \\
=\frac{1}{n !} \sum_{j=1}^{n} \sum_{\pi \in \mathfrak{S}_{n}} Q_{\pi}\left(x_{1}, \ldots, x_{n}\right) h\left(x_{\pi^{-1}(j)}\right) f^{(n)}\left(x_{\pi^{-1}(1)}, \ldots, x_{\pi^{-1}(n)}\right) \\
\quad \times p_{i_{1}}\left(s_{\pi^{-1}(1)}\right) \cdots p_{i_{j}+1}\left(s_{\pi^{-1}(j)}\right) \cdots p_{i_{n}}\left(s_{\pi^{-1}(n)}\right) \\
=\frac{1}{n !} \sum_{j=1}^{n} \sum_{\pi \in \mathfrak{S}_{n}} Q_{\pi}\left(x_{1}, \ldots, x_{n}\right) g_{j}^{(n)}\left(x_{\pi^{-1}(1)}, s_{\pi^{-1}(1)}, \ldots, x_{\pi^{-1}(n)}, s_{\pi^{-1}(n)}\right) .
\end{aligned}
$$

Здесь

$$
g_{j}^{(n)}\left(x_{1}, s_{1}, \ldots, x_{n}, s_{n}\right):=h\left(x_{j}\right) f^{(n)}\left(x_{1}, \ldots, x_{n}\right) p_{i_{1}}\left(s_{1}\right) \cdots p_{i_{j}+1}\left(s_{j}\right) \cdots p_{i_{n}}\left(s_{n}\right),
$$

$j=1, \ldots, n$. Следовательно, в силу $(4.25)$ и (4.26)

$$
\begin{aligned}
\left(d \Gamma\left(M_{h} \otimes A^{+}\right) \operatorname{Sym}_{n} F\right)\left(x_{1}, s_{1}, \ldots, x_{n}, s_{n}\right) & \\
= & \frac{1}{(n !)^{2}} \sum_{j=1}^{n} \sum_{\sigma \in \mathfrak{S}_{n}} \sum_{\pi \in \mathfrak{S}_{n}} Q_{\sigma}\left(x_{1}, \ldots, x_{n}\right) Q_{\pi}\left(x_{\sigma^{-1}(1)}, \ldots, x_{\sigma^{-1}(n)}\right) \\
& \quad \times g_{j}^{(n)}\left(x_{\sigma^{-1}\left(\pi^{-1}(1)\right)}, s_{\sigma^{-1}\left(\pi^{-1}(1)\right)}, \ldots, x_{\sigma^{-1}\left(\pi^{-1}(n)\right)}, s_{\sigma^{-1}\left(\pi^{-1}(n)\right)}\right) .
\end{aligned}
$$

Отсюда вытекает, что

$$
\begin{aligned}
d \Gamma\left(M_{h} \otimes A^{+}\right) \operatorname{Sym}_{n} F & =\sum_{j=1}^{n} \operatorname{Sym}_{n}^{2} g_{j}^{(n)}=\sum_{j=1}^{n} \operatorname{Sym}_{n} g_{j}^{(n)} \\
& =\operatorname{Sym}_{n}\left(\sum_{j=1}^{n} g_{j}^{(n)}\right)=\operatorname{Sym}_{n}\left(N\left(M_{h} \otimes A^{+}\right) F\right) .
\end{aligned}
$$

Для операторов $A^{0}, A^{-}$рассуждения аналогичны.

Теперь утверждение предложения 4.4 непосредственно следует из определения пространств $\mathbb{F}_{n}$, формул (4.16) и леммы 4.5. 
ПРЕДЛОЖЕНИЕ 4.6. Пустъ $h_{1}, \ldots, h_{n} \in C_{0}(X)$. Тогда

$$
I\left\langle P_{n}(\omega), h_{1} \otimes \cdots \otimes h_{n}\right\rangle=\mathscr{A}^{+}\left(h_{1}\right) \cdots \mathscr{A}^{+}\left(h_{n}\right) \Omega .
$$

ДокАЗАТЕльство. Напомним, что $\left\langle P_{n}(\omega), h_{1} \otimes \cdots \otimes h_{n}\right\rangle-$ это ортогональная проекция монома

$$
\left\langle h_{1}, \omega\right\rangle \cdots\left\langle h_{n}, \omega\right\rangle=\left\langle h_{1} \otimes \cdots \otimes h_{n}, \omega^{\otimes n}\right\rangle
$$

на $\mathscr{O} \mathscr{P}_{n}$. Требуемое утверждение вытекает из предложений 4.2 и 4.4, если заметить, что

$$
I\left\langle P_{n}(\omega), h_{1} \otimes \cdots \otimes h_{n}\right\rangle
$$

совпадает с ортогональной проекцией

$$
\begin{aligned}
\left\langle\omega, h_{1}\right\rangle & \cdots\left\langle\omega, h_{n}\right\rangle \Omega \\
= & \left(\mathscr{A}^{+}\left(h_{1}\right)+\mathscr{A}^{0}\left(h_{1}\right)+\mathscr{A}^{-}\left(h_{1}\right)\right) \cdots\left(\mathscr{A}^{+}\left(h_{n}\right)+\mathscr{A}^{0}\left(h_{n}\right)+\mathscr{A}^{-}\left(h_{n}\right)\right) \Omega
\end{aligned}
$$

на $\mathbb{F}_{n}$. Предложение доказано.

Теперь мы явно вычислим вектор $I\left\langle P_{n}(\omega), h_{1} \otimes \cdots \otimes h_{n}\right\rangle$. Для этого введем на $B_{0}\left(X^{n}\right)$ топологию, порождающую следующую сходимость: $f_{n} \rightarrow f$ при $n \rightarrow \infty$ тогда и только тогда, когда найдется такое множество $\Delta \in \mathscr{B}_{0}(X)$, что $\operatorname{supp}\left(f_{n}\right) \subset \Delta$ для всех $n \in \mathbb{N}$ и выполнено $(2.21)$. Отметим, что $C_{0}\left(X^{n}\right)-$ топологическое подпространство пространства $B_{0}\left(X^{n}\right)$.

Для каждого $\theta=\left\{\theta_{1}, \ldots, \theta_{l}\right\} \in \Pi(n)$, где $\theta_{1}, \ldots, \theta_{l}$ удовлетворяют условию (3.4), положим

$$
\left(\mathscr{E}_{\theta} f^{(n)}\right)\left(x_{1}, s_{1}, \ldots, x_{l}, s_{l}\right):=f_{\theta}^{(n)}\left(x_{1}, \ldots, x_{l}\right) p_{\left|\theta_{1}\right|-1}\left(s_{1}\right) p_{\left|\theta_{2}\right|-1}\left(s_{2}\right) \cdots p_{\left|\theta_{l}\right|-1}\left(s_{l}\right),
$$

где $f^{(n)} \in B_{0}\left(X^{n}\right),\left(x_{1}, \ldots, x_{l}\right) \in X^{(l)},\left(s_{1}, \ldots, s_{l}\right) \in \mathbb{R}^{l}$, а функция $f_{\theta}^{(n)}\left(x_{1}, \ldots, x_{l}\right)$ получается из функции $f^{(n)}\left(y_{1}, \ldots, y_{n}\right)$ заменой $y_{i_{1}}$ на $x_{1}$ для всех $i_{1} \in \theta_{1}$, заменой $y_{i_{2}}$ на $x_{2}$ для всех $i_{2} \in \theta_{2}$ и т. д. Отметим, что функция $f_{\theta}^{(n)}: X^{(l)} \rightarrow \mathbb{C}$ полностью определяется сужением функции $f^{(n)}: X^{n} \rightarrow \mathbb{C}$ на множество $X_{\theta}^{(n)}$.

$\mathrm{K}$ примеру, пусть $n=6$, а $\theta=\left\{\theta_{1}, \theta_{2}, \theta_{3}\right\} \in \Pi(6)$ имеют вид

$$
\theta_{1}=\{1,3\}, \quad \theta_{2}=\{2,4,6\}, \quad \theta_{3}=\{5\} .
$$

Тогда для любых $\left(x_{1}, x_{2}, x_{3}\right) \in X^{(3)}$ и $\left(s_{1}, s_{2}, s_{3}\right) \in \mathbb{R}^{3}$

$$
\left(\mathscr{E}_{\theta} f^{(6)}\right)\left(x_{1}, s_{1}, x_{2}, s_{2}, x_{3}, s_{3}\right)=f^{(6)}\left(x_{1}, x_{2}, x_{1}, x_{2}, x_{3}, x_{2}\right) p_{1}\left(s_{1}\right) p_{2}\left(s_{2}\right) p_{0}\left(s_{3}\right) .
$$

ПреДЛОЖениЕ 4.7. Для любого $n \in \mathbb{N}$ отображение

$$
\left(C_{0}(X)\right)^{n} \ni\left(h_{1}, \ldots, h_{n}\right) \mapsto\left\langle P_{n}(\omega), h_{1} \otimes \cdots \otimes h_{n}\right\rangle \in L^{2}(\tau)
$$

может быть продолжено линейно и непрерьвно до отображения

$$
B_{0}\left(X^{n}\right) \ni f^{(n)} \mapsto\left\langle P_{n}(\omega), f^{(n)}\right\rangle \in L^{2}(\tau) .
$$

Более того, при любом $f^{(n)} \in B_{0}\left(X^{n}\right)$

$$
I\left\langle P_{n}(\omega), f^{(n)}\right\rangle=\operatorname{Sym}\left(\sum_{\theta \in \Pi(n)} \mathscr{E}_{\theta} f^{(n)}\right) .
$$


ДокАЗАтЕльство. Зафиксируем произвольно $h_{1}, \ldots, h_{n} \in C_{0}(X)$ и положим $f^{(n)}\left(x_{1}, \ldots, x_{n}\right)=h_{1}\left(x_{1}\right) \cdots h_{n}\left(x_{n}\right)$. Тогда по предложению 4.6 формула (4.28) эквивалентна формуле

$$
\begin{aligned}
& \left(a^{+}\left(h_{1} \otimes 1\right)+d \Gamma\left(M_{h_{1}} \otimes A^{+}\right)\right) \cdots\left(a^{+}\left(h_{n} \otimes 1\right)+d \Gamma\left(M_{h_{n}} \otimes A^{+}\right)\right) \Omega \\
& \quad=\operatorname{Sym}\left(\sum_{\theta \in \Pi(n)} \mathscr{E}_{\theta} f^{(n)}\right)
\end{aligned}
$$

для доказательства которой, в силу (4.16) и леммы 4.5, достаточно показать, что

$$
\left(J^{+}\left(h_{1} \otimes 1\right)+N\left(M_{h_{1}} \otimes A^{+}\right)\right) \cdots\left(J^{+}\left(h_{n} \otimes 1\right)+N\left(M_{h_{n}} \otimes A^{+}\right)\right) \Omega=\sum_{\theta \in \Pi(n)} \mathscr{E}_{\theta} f^{(n)} .
$$

Докажем (4.30). Пусть $\beta=\left\{\beta_{1}, \ldots, \beta_{k}\right\}$ - (неупорядоченное) разбиение множества $\{i+1, i+2, \ldots, n\}$. Тогда

$$
J^{+}\left(h_{i} \otimes 1\right) \mathscr{E}_{\beta}\left(h_{i+1} \otimes h_{i+2} \otimes \cdots \otimes h_{n}\right)=\mathscr{E}_{\beta^{+}}\left(h_{i} \otimes h_{i+1} \otimes \cdots \otimes h_{n}\right),
$$

где $\beta^{+}:=\left\{\{i\}, \beta_{1}, \ldots, \beta_{k}\right\}$ - разбиение множества $\{i, i+1, \ldots, n\}$. Далее,

$$
N\left(M_{h_{i}} \otimes A^{+}\right) \mathscr{E}_{\beta}\left(h_{i+1} \otimes h_{i+2} \otimes \cdots \otimes h_{n}\right)=\sum_{j=1}^{k} \mathscr{E}_{\beta_{j}^{0}}\left(h_{i} \otimes h_{i+1} \otimes \cdots \otimes h_{n}\right),
$$

где $\beta_{j}^{0}$ - разбиение множества $\{i, i+1, \ldots, n\}$, полученное из $\beta$ добавлением элемента $i$ в множество $\beta_{j}$, т. е.

$$
\beta_{j}^{0}:=\left\{\beta_{1}, \ldots, \beta_{j} \cup\{i\}, \ldots, \beta_{k}\right\} .
$$

Теперь формула (4.30) следует из (4.31), (4.32) по индукции.

Наконец, формула (4.28) может быть обобщена на случай общих $f^{(n)} \in$ $B_{0}\left(X^{n}\right)$ по линейности и из соображений аппроксимации. Предложение доказано.

Теперь мы можем доказать теорему 3.2. На самом деле мы покажем немного больше, а именно что формула (3.10) имеет место при всех $f^{(n)}, g^{(n)} \in B_{0}\left(X^{n}\right)$.

Для начала заметим, что достаточно установить формулу (3.10) в случае, когда $f^{(n)}=g^{(n)}=h_{1} \otimes \cdots \otimes h_{n}$, где $h_{1}, \ldots, h_{n} \in B_{0}(X)$. По предложению 4.7

$$
\begin{aligned}
& \left(\left\langle P_{n}(\omega), f^{(n)}\right\rangle,\left\langle P_{n}(\omega), f^{(n)}\right\rangle\right)_{L^{2}(\tau)} \\
& =\left(\sum_{\theta \in \Pi(n)} \operatorname{Sym}_{|\theta|}\left(\mathscr{E}_{\theta} f^{(n)}\right), \sum_{\zeta \in \Pi(n)} \operatorname{Sym}_{|\zeta|}\left(\mathscr{E}_{\zeta} f^{(n)}\right)\right)_{\mathscr{F} Q(\mathscr{G})} \\
& =\sum_{l=1}^{n} \sum_{\substack{\theta, \zeta \in \Pi(n) \\
|\theta|=|\zeta|=l}}\left(\operatorname{Sym}_{l}\left(\mathscr{E}_{\theta} f^{(n)}\right), \mathscr{E}_{\zeta} f^{(n)}\right)_{L^{2}\left((X \times \mathbb{R})^{l},(m \otimes \nu)^{\otimes l}\right)} l !
\end{aligned}
$$


Далее, отметим, что по предложению 3.1

$$
\begin{aligned}
\left(\operatorname{Sym}_{n} f^{(n)}, \operatorname{Sym}_{n} f^{(n)}\right)_{\mathbf{F}_{n}^{Q}(\mathscr{H}, \nu)} & =\int_{X^{n}}\left(\operatorname{Sym}_{n} f^{(n)}\right) f^{(n)} d m_{\nu}^{(n)} \\
& =\sum_{\zeta \in \Pi(n)} \int_{X_{\zeta}^{(n)}}\left(\operatorname{Sym}_{n} f^{(n)}\right) f^{(n)} d m_{\nu, \zeta}^{(n)}
\end{aligned}
$$

С учетом (4.33), (4.34) формула (3.10) будет доказана, если мы покажем, что

$$
\begin{aligned}
& \sum_{\theta \in \Pi(n),|\theta|=l}\left(\operatorname{Sym}_{l}\left(\mathscr{E}_{\theta} f^{(n)}\right), \mathscr{E}_{\zeta} f^{(n)}\right)_{L^{2}\left((X \times \mathbb{R})^{l},(m \otimes \nu) \otimes l\right.} l ! \\
& =\int_{X_{\zeta}^{(n)}}\left(\operatorname{Sym}_{n} f^{(n)}\right) f^{(n)} d m_{\nu, \zeta}^{(n)}
\end{aligned}
$$

при фиксированном $\zeta \in \Pi(n),|\zeta|=l$.

Итак, зафиксируем разбиение $\zeta=\left\{\zeta_{1}, \ldots, \zeta_{l}\right\} \in \Pi(n)$ и предположим, что выполнено условие (4.6). Обозначим $k_{i}:=\left|\zeta_{i}\right|, i=1, \ldots, l$. По определению $\mathscr{E}_{\zeta} f^{(n)}$ имеем:

$$
\mathscr{E}_{\zeta} f^{(n)}=\left(\prod_{i_{1} \in \zeta_{1}} h_{i_{1}}\right) \otimes p_{k_{1}-1} \otimes \cdots \otimes\left(\prod_{i_{l} \in \zeta_{l}} h_{i_{l}}\right) \otimes p_{k_{l}-1}
$$

Пусть $\theta=\left\{\theta_{1}, \ldots, \theta_{l}\right\} \in \Pi(n)$ и выполнено условие (3.4). Для $r_{i}:=\left|\theta_{i}\right|, i=$ $1, \ldots, l$, мы можем считать, что найдется перестановка $\widehat{\pi} \in \mathfrak{S}_{l}$ такая, что

$$
r_{i}=k_{\widehat{\pi}(i)}, \quad i=1, \ldots, l
$$

(действительно, в противном случае соответствующее слагаемое в левой части формулы (4.35) равно нулю). Аналогично (4.36) имеем

$$
\begin{aligned}
& l ! \operatorname{Sym}_{l}\left(\mathscr{E}_{\theta} f^{(n)}\right)\left(y_{1}, s_{1}, \ldots, y_{l}, s_{l}\right)=\sum_{\varkappa \in S_{l}} Q_{\varkappa}\left(y_{1}, \ldots, y_{l}\right) \\
& \times\left(\left(\prod_{j_{1} \in \theta_{\varkappa(1)}} h_{j_{1}}\right) \otimes p_{r_{\varkappa(1)}-1} \otimes \cdots \otimes\left(\prod_{j_{l} \in \theta_{\varkappa(l)}} h_{j_{l}}\right) \otimes p_{r_{\varkappa(l)}-1}\right)\left(y_{1}, s_{1}, \ldots, y_{l}, s_{l}\right) .
\end{aligned}
$$

Отсюда, применяя (3.3), находим, что

$$
\begin{aligned}
&\left(\operatorname{Sym}_{l}\left(\mathscr{E}_{\theta} f^{(n)}\right), \mathscr{E}_{\zeta} f^{(n)}\right)_{L^{2}\left((X \times \mathbb{R})^{l},(m \otimes \nu)^{\otimes l}\right)^{l}} l ! \\
&=\sum_{\widehat{\pi}} \int_{X^{l}} Q_{\widehat{\pi}}\left(y_{1}, \ldots, y_{l}\right)\left(\prod_{j_{1} \in \theta_{\widehat{\pi}(1)}} h_{j_{1}}\left(y_{1}\right)\right)\left(\prod_{i_{1} \in \zeta_{1}} h_{i_{1}}\left(y_{1}\right)\right) \times \cdots \\
& \quad \times\left(\prod_{j_{l} \in \theta_{\widehat{\pi}(l)}} h_{j_{l}}\left(y_{l}\right)\right)\left(\prod_{i_{l} \in \zeta_{l}} h_{i_{l}}\left(y_{l}\right)\right) d y_{1} \cdots d y_{l} c_{k_{1}} \cdots c_{k_{l}}
\end{aligned}
$$


где сумма берется по всем перестановкам $\widehat{\pi} \in S_{l}$, удовлетворяющим (4.37). Зафиксируем такую перестановку $\widehat{\pi}$. Тогда найдется ровно

$$
r_{1} ! \cdots r_{l} !=k_{1} ! \cdots k_{l} !
$$

перестановок $\pi \in \mathfrak{S}_{n}$ таких, что

$$
\pi \zeta_{i}=\theta_{\widehat{\pi}(i)}, \quad i=1, \ldots, l
$$

Заметим, что

$$
\begin{aligned}
f^{(n)}\left(x_{\pi^{-1}(1)}, \ldots, x_{\pi^{-1}(n)}\right) & =\left(h_{1} \otimes \cdots \otimes h_{n}\right)\left(x_{\pi^{-1}(1)}, \ldots, x_{\pi^{-1}(n)}\right) \\
& =\left(h_{\pi(1)} \otimes \cdots \otimes h_{\pi(n)}\right)\left(x_{1}, \ldots, x_{n}\right) \\
& =\left(\prod_{j_{1} \in \pi \zeta_{1}} h_{j_{1}}\right)\left(y_{1}\right) \cdots\left(\prod_{j_{l} \in \pi \zeta_{l}} h_{j_{l}}\right)\left(y_{l}\right) \\
& =\left(\prod_{j_{1} \in \theta_{\hat{\pi}(1)}} h_{j_{1}}\right)\left(y_{1}\right) \cdots\left(\prod_{j_{l} \in \theta_{\hat{\pi}(l)}} h_{j_{l}}\right)\left(y_{l}\right)
\end{aligned}
$$

для любой перестановки $\pi$, удовлетворяющей $(4.39)$, и любого $\left(x_{1}, \ldots, x_{n}\right) \in X_{\zeta}^{(n)}$, где

$$
y_{1}=x_{i_{1}} \quad \text { при } i_{1} \in \zeta_{1}, \quad \ldots, \quad y_{l}=x_{i_{l}} \quad \text { при } i_{l} \in \zeta_{l} .
$$

Пусть $\zeta, \theta \in \Pi(n)$ таковы, что условие (4.37) выполнено для некоторой перестановки $\widehat{\pi} \in \mathfrak{S}_{l}$. Иными словами, соответствующие последовательности $\left(k_{1}, \ldots, k_{l}\right)$ и $\left(r_{1}, \ldots, r_{l}\right)$ совпадают с точностью до перестановки. Через $\mathfrak{S}_{n}[\zeta, \theta]$ обозначим множество всех перестановок $\pi \in \mathfrak{S}_{n}$, которые удовлетворяют (4.39) с некоторой перестановкой $\widehat{\pi} \in \mathfrak{S}_{l}$. Отметим, что перестановка $\widehat{\pi}$ полностью определяется по $\pi, \zeta$ и $\theta$ и при этом автоматически удовлетворяет (4.39). Ясно, что если $\theta$ и $\theta^{\prime},|\theta|=\left|\theta^{\prime}\right|=l, \theta \neq \theta^{\prime}$, лежат в П $(n)$ и для них выполнено условие (4.39), то

$$
\mathfrak{S}_{n}[\zeta, \theta] \cap \mathfrak{S}_{n}\left[\zeta, \theta^{\prime}\right]=\varnothing
$$

Далее,

$$
\bigcup_{\substack{\theta \in \Pi(n),|\theta|=l \\ \theta \text { удовлетворяет }(4.39)}} \mathfrak{S}_{n}[\zeta, \theta]=\mathfrak{S}_{n} .
$$

Следовательно, используя определение меры $m_{c, \zeta}^{(n)}$ и принимая во внимание формулы (3.7), (4.38), (4.40)-(4.42), находим, что

$$
\begin{aligned}
\left.\left(\operatorname{Sym}_{l}\left(\mathscr{E}_{\theta} f^{(n)}\right), \mathscr{E}_{\zeta} f^{(n)}\right)_{L^{2}\left((X \times \mathbb{R})^{l},(m \otimes \nu)\right.}^{\otimes l}\right) & l ! \\
= & \frac{1}{n !} \sum_{\pi \in S_{n}[\zeta, \theta]} \int_{X_{\zeta}^{(n)}} Q_{\pi}\left(x_{1}, \ldots, x_{n}\right) f^{(n)}\left(x_{\pi^{-1}(1)}, \ldots, x_{\pi^{-1}(n)}\right) \\
& \times f^{(n)}\left(x_{1}, \ldots, x_{n}\right) m_{\nu, \zeta}^{(n)}\left(d x_{1} \times \cdots \times d x_{n}\right) .
\end{aligned}
$$


Отсюда получаем

$$
\begin{aligned}
& \sum_{\theta \in \Pi(n),|\theta|=l}\left(\operatorname{Sym}_{l}\left(\mathscr{E}_{\theta} f^{(n)}\right), \mathscr{E}_{\zeta} f^{(n)}\right)_{L^{2}\left((X \times \mathbb{R})^{l},(m \otimes \nu)^{\otimes l}\right)} l ! \\
& =\sum_{\substack{\theta \in \Pi(n),|\theta|=l \\
\theta \text { удовлетворяет }(4.39)}}\left(\operatorname{Sym}_{l}\left(\mathscr{E}_{\theta} f^{(n)}\right), \mathscr{E}_{\zeta} f^{(n)}\right)_{L^{2}\left((X \times \mathbb{R})^{l},(m \otimes \nu)^{\otimes l}\right)} l ! \\
& =\frac{1}{n !} \sum_{\substack{\theta \in \Pi(n),|\theta|=l \\
\theta \text { удовлетворяет }(4.39)}} \sum_{\pi \in S_{n}[\zeta, \theta]} \int_{X_{\zeta}^{(n)}} Q_{\pi}\left(x_{1}, \ldots, x_{n}\right) \\
& \quad \times f^{(n)}\left(x_{\pi^{-1}(1)}, \ldots, x_{\pi^{-1}(n)}\right) f^{(n)}\left(x_{1}, \ldots, x_{n}\right) m_{\nu, \zeta}^{(n)}\left(d x_{1} \times \cdots \times d x_{n}\right) \\
& \quad \frac{1}{n !} \sum_{\pi \in S_{n}} \int_{X_{\zeta}^{(n)}} Q_{\pi}\left(x_{1}, \ldots, x_{n}\right) f^{(n)}\left(x_{\pi^{-1}(1)}, \ldots, x_{\pi^{-1}(n)}\right) \\
& \quad \times f^{(n)}\left(x_{1}, \ldots, x_{n}\right) m_{\nu, \zeta}^{(n)}\left(d x_{1} \times \cdots \times d x_{n}\right) \\
& =\int_{X_{\zeta}^{(n)}}\left(\operatorname{Sym}_{n} f^{(n)}\right) f^{(n)} d m_{\nu, \zeta}^{(n)},
\end{aligned}
$$

что доказывает теорему 3.2 .

4.3. Доказательство теоремы 3.4. Сначала мы установим следующий результат.

Лемма 4.8. Пусть $h \in C_{0}(X)$ u $f^{n} \in B_{0}\left(X^{n}\right), n \in \mathbb{N}$. Тогда имеют место представления (3.13), (3.20), где

$$
\begin{aligned}
\mathbf{J}^{+}(h) \operatorname{Sym}_{n} f^{(n)} & =\operatorname{Sym}_{n+1}\left(h \otimes f^{(n)}\right), \\
\mathbf{J}^{0}(h) \operatorname{Sym}_{n} f^{(n)} & =\operatorname{Sym}_{n}\left(\mathscr{J}^{0}(h) f^{(n)}\right), \\
\mathbf{J}_{1}^{-}(h) \operatorname{Sym}_{n} f^{(n)} & =\operatorname{Sym}_{n-1}\left(\mathscr{J}_{1}^{-}(h) f^{(n)}\right), \\
\mathbf{J}_{2}^{-}(h) \operatorname{Sym}_{n} f^{(n)} & =\operatorname{Sym}_{n-1}\left(\mathscr{J}_{2}^{-}(h) f^{(n)}\right) .
\end{aligned}
$$

Здесъ

$$
\begin{aligned}
& \left(\mathscr{J}_{2}^{-}(h) f^{(n)}\right)\left(x_{1}, \ldots, x_{n-1}\right):=\sum_{i=1}^{n} \int_{X} d y h(y) \\
& \quad \times f^{(n)}\left(x_{1}, \ldots, x_{i-1}, y, x_{i}, \ldots, x_{n-1}\right) T_{i}\left(y, x_{1}, \ldots, x_{n-1}\right),
\end{aligned}
$$

где для любого $\theta \in \Pi(n-1)$

$$
T_{i}^{(n)}\left\lceil X \times X_{\theta}^{(n-1)}:=\prod_{\theta_{u} \in \theta: \max \theta_{u} \leqslant i-1} Q\left(y, x_{\theta_{u}}\right) .\right.
$$


ДокАЗАТЕЛЬСтво. Используя (4.22) и (4.23), находим, что

$$
\langle\omega, h\rangle=\mathscr{A}^{+}(h)+\mathscr{A}^{0}(h)+d \Gamma\left(M_{h} \otimes A^{-}\right)+a^{-}(h \otimes 1) .
$$

(i) (часть, относящаяся $\kappa \mathbf{J}^{+}(h)$ ). Из рассуждений, использованных при доказательстве предложения 4.7 , следует, что

$$
\mathbf{U}^{-1} \mathscr{A}^{+}(h) \mathbf{U} \operatorname{Sym}_{n} f^{(n)}=\operatorname{Sym}_{n+1}\left(h \otimes f^{(n)}\right)=\mathbf{J}^{+}(h) \operatorname{Sym}_{n} f^{(n)} .
$$

(ii) (часть, относящаяся $\kappa \mathbf{J}^{0}(h)$ ). Используя лемму 4.5, предложение 4.7, а также соотношения (4.15), (4.23), (3.15), (3.16), находим, что

$$
\begin{aligned}
\mathbf{U}^{-1} \mathscr{A}^{0}(h) \mathbf{U S y m}_{n} f^{(n)} & =\mathbf{U}^{-1} \mathscr{A}^{0}(h) \operatorname{Sym}\left(\sum_{\theta \in \Pi(n)} \mathscr{E}_{\theta} f^{(n)}\right) \\
& =\mathbf{U}^{-1} \operatorname{Sym}\left(N\left(M_{h} \otimes A^{0}\right) \sum_{\theta \in \Pi(n)} \mathscr{E}_{\theta} f^{(n)}\right) \\
& =\mathbf{U}^{-1} \operatorname{Sym}\left(\sum_{\theta \in \Pi(n)} \sum_{i=1}^{n} \mathscr{E}_{\theta}\left(h \times_{i} f^{(n)}\right) b_{\gamma(i, \theta)-1} \gamma(i, \theta)^{-1}\right) \\
& =\operatorname{Sym}_{n}\left(\mathscr{J}^{0}(h) f^{(n)}\right) \\
& =\mathbf{J}^{0}(h) \operatorname{Sym}_{n} f^{(n)} .
\end{aligned}
$$

Здесь

$$
\left(h \times_{i} f^{(n)}\right)\left(x_{1}, \ldots, x_{n}\right):=h\left(x_{i}\right) f^{(n)}\left(x_{1}, \ldots, x_{n}\right) .
$$

(iii) (часть, относящаяся $\left.\kappa \mathbf{J}_{1}^{-}(h)\right)$. Как и выше,

$$
\begin{aligned}
& \mathbf{U}^{-1} d \Gamma\left(M_{h} \otimes A^{-}\right) \mathbf{U S y m}_{n} f^{(n)}=\mathbf{U}^{-1} \operatorname{Sym}\left(N\left(M_{h} \otimes A^{-}\right) \sum_{\theta \in \Pi(n)} \mathscr{E}_{\theta} f^{(n)}\right) \\
& =\mathbf{U}^{-1} \operatorname{Sym}\left(\sum_{l=1}^{n} \sum_{\substack{\theta \in \Pi(n) \\
|\theta|=l}} \sum_{k=1}^{l} \mathbf{1}^{\otimes(k-1)} \otimes\left(M_{h} \otimes A^{-}\right) \otimes \mathbf{1}^{\otimes(l-k)} \mathscr{E}_{\theta} f^{(n)}\right) \\
& =\mathbf{U}^{-1} \operatorname{Sym}\left(\sum_{l=1}^{n-1} \sum_{\theta=\left\{\theta_{1}, \ldots, \theta_{l}\right\} \in \Pi(n)} \sum_{\substack{1 \leqslant k \leqslant l \\
\left|\theta_{k}\right| \geqslant 2}} \mathbf{1}^{\otimes(k-1)} \otimes\left(M_{h} \otimes A^{-}\right) \otimes \mathbf{1}^{\otimes(l-k)} \mathscr{E}_{\theta} f^{(n)}\right),
\end{aligned}
$$

где предполагается, что выполнено (3.4). Отметим, что при $\theta=\left\{\theta_{1}, \ldots, \theta_{l}\right\} \in$ $\Pi(n)$, удовлетворяющих $(3.4)$, и $k \in\{1, \ldots, l\}$ таких, что $\left|\theta_{k}\right| \geqslant 2$, имеем

$$
\begin{aligned}
\left(\mathbf{1}^{\otimes(k-1)} \otimes\left(M_{h} \otimes A^{-}\right) \otimes \mathbf{1}^{\otimes(l-k)} \mathscr{E}_{\theta} f^{(n)}\right)\left(x_{1}, s_{1}, \ldots, x_{l}, s_{l}\right) \\
=a_{\left|\theta_{k}\right|-1} h\left(x_{k}\right) f_{\theta}^{(n)}\left(x_{1}, \ldots, x_{k}, \ldots, x_{l}\right) p_{\left|\theta_{1}\right|-1}\left(s_{1}\right) \cdots p_{\left|\theta_{k-1}\right|-1}\left(s_{k-1}\right) \\
\quad \times p_{\left|\theta_{k}\right|-2}\left(s_{k}\right) p_{\left|\theta_{k+1}\right|-1}\left(s_{k+1}\right) \cdots p_{\left|\theta_{l}\right|-1}\left(s_{l}\right) .
\end{aligned}
$$

Зафиксируем произвольно $i, j \in\{1, \ldots, n\}, i<j$, и рассмотрим множество

$$
L_{i}:=\{1,2, \ldots, i-1, i+1, \ldots, n\},
$$


состоящее из $n-1$ элемента. Тогда любое разбиение $\zeta=\left\{\zeta_{1}, \ldots, \zeta_{l}\right\} \in \Pi(n-1)$ определяет разбиение $\widetilde{\zeta}=\left\{\widetilde{\zeta}_{1}, \ldots, \widetilde{\zeta}_{l}\right\}$ множества $L_{i}: \widetilde{\zeta}_{u}:=K_{i} \zeta_{u}, u=1, \ldots, l$, где

$$
K_{i} v:= \begin{cases}v, & \text { если } v \leqslant i-1, \\ v+1, & \text { если } v \geqslant i .\end{cases}
$$

Пусть $\widetilde{\zeta}_{k}-$ элемент разбиения $\widetilde{\zeta}$, содержащий $j$. Положим

$$
\theta_{u}:= \begin{cases}\widetilde{\zeta}_{u}, & \text { если } u \neq k, \\ \widetilde{\zeta}_{k} \cup\{i\}, & \text { если } u=k .\end{cases}
$$

Таким образом, мы построили разбиение $\theta=\left\{\theta_{1}, \ldots, \theta_{l}\right\} \in \Pi(n)$, где $l \leqslant n-1$. Далее, рассмотрим произвольное разбиение $\theta=\left\{\theta_{1}, \ldots, \theta_{l}\right\} \in \Pi(n), l \leqslant n-1$. Выберем произвольно $k \in\{1, \ldots, l\}$ такое, что $\left|\theta_{k}\right| \geqslant 2$. Возникает вопрос: сколькими способами можно получить $\theta$ из $i, j$ и $\zeta \in \Pi(n-1)$ аналогично тому, как это было сделано выше? Очевидно, что это число равно числу способов выбрать числа $i, j \in\{1, \ldots, n\}$, удовлетворяющие условиям $i<j$ и $i, j \in \theta_{k}$, т. е.

$$
\frac{1}{2}\left|\theta_{k}\right|\left(\left|\theta_{k}\right|-1\right)=\frac{1}{2}\left(\left|\widetilde{\zeta}_{k}\right|+1\right)\left|\widetilde{\zeta}_{k}\right|=\frac{1}{2}\left(\left|\zeta_{k}\right|+1\right)\left|\zeta_{k}\right|
$$

где $j \in \widetilde{\zeta}_{k}$ (или, эквивалентно, $j-1 \in \zeta_{k}$ ). Отсюда с учетом (3.4), (3.17), (3.18), (4.48) и (4.49) мы заключаем, что

$$
\mathbf{U}^{-1} d \Gamma\left(M_{h} \otimes A^{-}\right) \mathbf{U S y m}_{n} f^{(n)}=\operatorname{Sym}_{n-1}\left(\mathscr{J}_{1}^{-}(h) f^{(n)}\right)=\mathbf{J}_{1}^{-}(h) \operatorname{Sym}_{n} f^{(n)} .
$$

(iv) (часть, относящаяся $\left.\kappa \mathbf{J}_{2}^{-}(h)\right)$. Для любого разбиения $\theta=\left\{\theta_{1}, \ldots, \theta_{l}\right\} \in$ $\Pi(n)$, удовлетворяющего (3.4), имеем

$$
\begin{aligned}
&\left(a^{-}(h\right.\left.\otimes 1) \operatorname{Sym}_{l}\left(\mathscr{E}_{\theta} f^{(n)}\right)\right)\left(x_{1}, s_{1}, \ldots, x_{l-1}, s_{l-1}\right) \\
& \quad= \operatorname{Sym}_{l-1}\left(\int_{X} d y \sum_{\substack{1 \leqslant i \leqslant l \\
\left|\theta_{i}\right|=1}} h(y) Q\left(y, x_{1}\right) Q\left(y, x_{2}\right) \cdots Q\left(y, x_{i-1}\right)\right. \\
& \quad \times f_{\theta}^{(n)}\left(x_{1}, \ldots, x_{i-1}, y, x_{i}, \ldots, x_{l-1}\right) \\
&\left.\quad \times p_{\left|\theta_{1}\right|-1}\left(s_{1}\right) \cdots p_{\left|\theta_{i-1}\right|-1}\left(s_{i-1}\right) p_{\left|\theta_{i+1}\right|-1}\left(s_{i}\right) \cdots p_{\left|\theta_{l}\right|-1}\left(s_{l-1}\right)\right),
\end{aligned}
$$

где мы воспользовались соотношениями (2.7) и (4.27). Отсюда и из (4.43), (4.44) получаем

$$
\mathbf{U}^{-1} a^{-}(h \otimes 1) \mathbf{U} \operatorname{Sym}_{n} f^{(n)}=\operatorname{Sym}_{n-1}\left(\mathscr{J}_{2}^{-}(h) f^{(n)}\right)=\mathbf{J}_{2}^{-}(h) \operatorname{Sym}_{n} f^{(n)} .
$$

Лемма доказана.

Лемма 4.9. Пусть $h \in C_{0}(X) u f^{(n)} \in \mathbf{B}_{0}^{Q}\left(X^{n}\right)$. Тогда

$$
\begin{aligned}
\left(\mathbf{J}_{2}^{-}(h) f^{(n)}\right)\left(x_{1}, \ldots, x_{n-1}\right) & =\left(\mathscr{J}_{2}^{-}(h) f^{(n)}\right)\left(x_{1}, \ldots, x_{n-1}\right) \\
& =n \int_{X} d y h(y) f^{(n)}\left(y, x_{1}, \ldots, x_{n-1}\right) .
\end{aligned}
$$


ДокАЗАтЕльство. Зафиксируем произвольно $n \geqslant 2$ и $i \in\{2, \ldots, n\}$. Пусть перестановка $\pi \in \mathfrak{S}_{n}$ задана следующим образом: $\pi(1)=i, \pi(j)=j-1$ при $j=2, \ldots, i$ и $\pi(j)=j$ при $j=i+1, \ldots, n$. Если $j \in\{2, \ldots, n\}$, то из (3.7), (4.44) для всех $\left(x_{1}, \ldots, x_{n}\right) \in X^{n}$, у которых $x_{1} \neq x_{j}$, получаем

$$
\left(\Psi_{\pi} f^{(n)}\right)\left(x_{1}, \ldots, x_{n}\right)=f^{(n)}\left(x_{2}, x_{3}, \ldots, x_{i}, x_{1}, x_{i+1}, \ldots, x_{n}\right) T_{i}\left(x_{1}, x_{2}, \ldots, x_{n}\right),
$$

где оператор $\Psi_{\pi}$ определен формулой (4.1).

Tак как $f \in \mathbf{B}_{0}^{Q}\left(X^{n}\right)$, то в силу (4.5) и (4.10)

$$
\Psi_{\pi} f^{(n)}=\Psi_{\pi} \operatorname{Sym}_{n} f^{(n)}=\operatorname{Sym}_{n} f^{(n)}=f^{(n)} .
$$

Далее, из (4.54) и (4.55) вытекает, что при $\left(x_{1}, \ldots, x_{n-1}\right) \in X^{n-1}$

$$
\begin{aligned}
\int_{X} d y & h(y) f^{(n)}\left(x_{1}, \ldots, x_{i-1}, y, x_{i}, \ldots, x_{n-1}\right) T_{i}\left(y, x_{1}, \ldots, x_{n-1}\right) \\
& =\int_{X \backslash\left\{x_{1}, \ldots, x_{n-1}\right\}} d y h(y) f^{(n)}\left(x_{1}, \ldots, x_{i-1}, y, x_{i}, \ldots, x_{n-1}\right) T_{i}\left(y, x_{1}, \ldots, x_{n-1}\right) \\
& =\int_{X} d y h(y) f^{(n)}\left(y, x_{1}, \ldots, x_{n-1}\right) .
\end{aligned}
$$

Отсюда, принимая во внимание (4.43), получаем

$$
\begin{aligned}
\left(\mathscr{J}_{2}^{-}(h) f^{(n)}\right)\left(x_{1}, \ldots, x_{n-1}\right) & =n \int_{X} d y h(y) f^{(n)}\left(y, x_{1}, \ldots, x_{n-1}\right) \\
& =: g^{(n-1)}\left(x_{1}, \ldots, x_{n-1}\right) .
\end{aligned}
$$

Так как $f^{(n)} \in \mathbf{B}_{0}^{Q}\left(X^{n}\right)$, то формула (3.8) выполнена при любом $\pi \in \mathfrak{S}_{n}$. Как следствие, для каждого $\pi \in \mathfrak{S}_{n-1}$ имеем

$$
g^{(n-1)}\left(x_{1}, \ldots, x_{n-1}\right)=Q_{\pi}\left(x_{1}, \ldots, x_{n-1}\right) g^{(n)}\left(x_{\pi^{-1}(1)}, \ldots, x_{\pi^{-1}(n)}\right)
$$

(см. (3.7)). Следовательно,

$$
\operatorname{Sym} g^{(n-1)}=g^{(n-1)} .
$$

Окончательно, лемма следует из (4.56) и (4.57).

Теперь теорема 3.4 получается применением лемм 4.8, 4.9.

4.4. Доказательство теоремы 3.5. Предположим, что выполнено (3.22). Тогда $R_{i}^{(n)} \equiv \lambda$ и $S_{j-1}^{(n)} \equiv 2 \eta$ в силу (3.16) и (3.18). Отсюда с учетом $(3.15),(3.17)$ вытекает, что при любом $h \in C_{0}(X)$ операторы $\mathscr{J}^{0}(h)$ и $\mathscr{J}_{1}^{-}(h)$ отображают $\mathscr{F}_{\text {fin }}\left(C_{0}(X)\right)$ в себя. Как следствие, условие $(\mathrm{C})$ выполнено. Более того, равенство (3.23) вытекает из теоремы 3.4.

Чтобы показать, что (3.22) необходимо для выполнения условия (C), мы будем действовать следующим образом. Сначала предположим, что мера сосредоточена в одной точке, т. е. $\nu=\delta_{\lambda}$ при некотором $\lambda \in \mathbb{R}$ (т. е. имеет место гауссовский или пуассоновский случай). Тогда $a_{k}=0$ для всех $k \in \mathbb{N}$ и $b_{0}=\lambda$, 
поэтому значения $b_{k}, k \in \mathbb{N}$, могут быть выбраны произвольно. Итак, мы показали, что в этом случае (3.22) выполнено при $\eta=0$.

Далее будем предполагать, что носитель меры $\nu$ содержит бесконечное число точек. Тогда $a_{k}>0$ для всех $k \in \mathbb{N}$.

Лемма 4.10. Пусть $q \neq-1$, и пусть $a_{k}>0$ при всех $k \in \mathbb{N}$. Далее, предположим, что $n \geqslant 2$ и функиии $f^{(n)} \in C_{0}\left(X^{n}\right)$ mаковы, что $\operatorname{Sym}_{n} f^{(n)}=0$ $m_{\nu}^{(n)}$-n.в. на множестве $X_{\theta}^{(n)}$, где $\theta=\left\{\theta_{1}, \theta_{2}\right\} \in \Pi(n), \theta_{1}=\{1\}, \theta_{2}=\{2, \ldots, n\}$. Тогда $f^{(n)}(x, \ldots, x)=0$ для всех $x \in X$.

В фермионном случае $(q=-1)$ данный результат выполнен при $n \geqslant 3$.

ДоказАтельство. Пусть $x_{1}, x_{2} \in X, x_{1}^{1}<x_{2}^{1}$. (Напомним, что $x^{i}$ обозначает $i$-ю координату вектора $x=\left(x^{1}, \ldots, x^{d}\right) \in X$.) В частности, $x_{1}<x_{2}$. Тогда

$$
\begin{aligned}
& \left(\operatorname{Sym}_{n} f^{(n)}\right)\left(x_{1}, x_{2}, x_{2}, \ldots, x_{2}\right)=\frac{1}{n}\left(f^{(n)}\left(x_{1}, x_{2}, x_{2}, \ldots, x_{2}\right)\right. \\
& \quad+f^{(n)}\left(x_{2}, x_{1}, x_{2}, \ldots, x_{2}\right)+\cdots \\
& \left.\quad+f^{(n)}\left(x_{2}, \ldots, x_{2}, x_{1}, x_{2}\right)+q f^{(n)}\left(x_{2}, \ldots, x_{2}, x_{1}\right)\right)=0 .
\end{aligned}
$$

Так как функция $f^{(n)}$ непрерывна, то равенство (4.58) выполнено поточечно на открытом множестве

$$
\left\{\left(x_{1}, x_{2}\right) \in X^{2} \mid x_{1}^{1}<x_{2}^{1}\right\} .
$$

Отсюда следует, что $((n-1+q) / n) f^{(n)}(x, \ldots, x)=0$ для всех $x \in X$. Таким образом, $f^{(n)}(x, \ldots, x)=0$ в случае, если $q \neq-1$ и $n \geqslant 2$ или если $q=-1$ и $n \geqslant 3$. Лемма доказана.

Теперь положим $\lambda:=b_{0}$. Наша цель - показать, что если условие $(\mathrm{C})$ выполнено, то $b_{k}=\lambda(k+1)$ при всех $k \in \mathbb{Z}_{+}$. Приводимое ниже доказательство проходит для любой энионной статистики, однако в случае $q \neq-1$ рассуждение можно существенно упростить.

Пусть $\varepsilon \in \mathbb{R}$ таково, что $b_{1}=2 \lambda+\varepsilon$. Мы покажем по индукции, что

$$
b_{k}=\lambda(k+1)+\varepsilon, \quad k \geqslant 1 .
$$

Предположим, что равенство (4.59) имеет место при всех $k=1, \ldots, n$. Зафиксируем произвольно $h \in C_{0}(X)$ и $f^{(n+2)} \in C_{0}\left(X^{n+2}\right)$ и определим функцию $g^{(n+2)} \in C_{0}\left(X^{n+2}\right)$ следующим образом:

$$
g^{(n+2)}\left(x_{1}, \ldots, x_{n+2}\right):=f^{(n+2)}\left(x_{1}, \ldots, x_{n+2}\right)\left(\lambda h\left(x_{1}\right)+h\left(x_{2}\right)(\lambda(n+1)+\varepsilon)\right) .
$$

Пусть $\theta=\left\{\theta_{1}, \theta_{2}\right\} \in \Pi(n+2)$, где $\theta_{1}=\{1\}, \theta_{2}=\{2, \ldots, n+2\}$. Из (3.15), (3.16) следует, что

$$
\begin{aligned}
& \left(\mathscr{J}^{0}(h) f^{(n+2)}\right)\left(x_{1}, \ldots, x_{n+2}\right) \\
& \quad=f^{(n+2)}\left(x_{1}, \ldots, x_{n+2}\right)\left(\lambda h\left(x_{1}\right)+(n+1) \frac{h\left(x_{2}\right)(\lambda(n+1)+\varepsilon)}{n+1}\right) \\
& \quad=g^{(n+2)}\left(x_{1}, \ldots, x_{n+2}\right)
\end{aligned}
$$


$m_{\nu}^{(n+2)}$-п. в. на $X_{\theta}^{(n+2)}$. Так как условие $(\mathrm{C})$ выполнено, то найдется функция $u^{(n+2)} \in C_{0}\left(X^{n+2}\right)$ такая, что

$$
\operatorname{Sym}_{n+2}\left(\mathscr{J}^{0}(h) f^{(n+2)}\right)=\operatorname{Sym}_{n+2} u^{(n+2)}
$$

$m_{\nu}^{(n+2)}$-п. в. на $X^{n+2}$. Отсюда следует, что

$$
\operatorname{Sym}_{n+2}\left(g^{(n+2)}-u^{(n+2)}\right)\left(x_{1}, \ldots, x_{n+2}\right)=0
$$

для $m_{\nu}^{(n+2)}$-почти всех $\left(x_{1}, \ldots, x_{n+2}\right) \in X_{\theta}^{(n+2)}$. Принимая во внимание, что $g^{(n+2)}-u^{(n+2)} \in C_{0}\left(X^{n+2}\right)$, мы заключаем из леммы 4.10, что

$$
u^{(n+2)}(x, \ldots, x)=g^{(n+2)}(x, \ldots, x), \quad x \in X .
$$

Применяя (4.60)-(4.62), получаем, что

$$
\left(\mathscr{J}^{0}(h) f^{(n+2)}\right)(x, \ldots, x)=(\lambda(n+2)+\varepsilon) h(x) f^{(n+2)}(x, \ldots, x)
$$

для всех $x \in X$. Далее, из (3.15), (3.16), (4.63) мы имеем $b_{n+1}=\lambda(n+2)+\varepsilon$. Таким образом, (4.59) доказано.

Нашей дальнейшей целью является доказательство равенства $\varepsilon=0$. Для начала мы получим следующий аналог леммы 4.10.

Лемма 4.11. Предположим, что $a_{k}>0$ для всех $k \in \mathbb{N}$, u nусть $f^{(5)} \in$ $C_{0}\left(X^{5}\right)$ такова, что $\mathrm{Sym}_{5} f^{(5)}=0 m_{\nu}^{(5)}-n$. в. на множестве $X_{\theta}^{(5)}$, где $\theta=$ $\left\{\theta_{1}, \theta_{2}\right\} \in \Pi(5), \theta_{1}=\{1,2\}, \theta_{2}=\{3,4,5\}$. Тогда $f^{(5)}(x, \ldots, x)=0$ для всех $x \in X$.

ДокАЗАТЕльство. Мы рассуждаем аналогично доказательству леммы 4.10. Действительно, из условия леммы 4.11 мы имеем $\frac{6+4 q}{10} f^{(5)}(x, \ldots, x)=0$, что дает требуемое утверждение.

Используя (3.15), (3.16), (4.59), получаем, что

$$
\left(\mathscr{J}^{0}(h) f^{(5)}\right)\left(x_{1}, \ldots, x_{5}\right)=f^{(5)}\left(x_{1}, \ldots, x_{5}\right)\left(h\left(x_{1}\right)(2 \lambda+\varepsilon)+h\left(x_{3}\right)(3 \lambda+\varepsilon)\right)
$$

для $m_{\nu}^{(5)}$-почти всех $\left(x_{1}, \ldots, x_{5}\right) \in X_{\theta}^{(5)}$, где $\theta \in \Pi(5)$ определено в лемме 4.11.

Аналогично выводу формулы (4.63), из условия (C), леммы 4.11 и соотношения (4.64) мы заключаем, что для всех $x \in X$

$$
\left(\mathscr{J}^{0}(h) f^{(5)}\right)(x, \ldots, x)=f^{(5)}(x, \ldots, x) h(x)(5 \lambda+2 \varepsilon) .
$$

С другой стороны, применяя (3.15), (3.16), (4.59), находим, что при всех $x \in X$

$$
\left(\mathscr{J}^{0}(h) f^{(5)}\right)(x, \ldots, x)=f^{(5)}(x, \ldots, x) h(x)(5 \lambda+\varepsilon) .
$$

Сравнивая (4.65) и (4.66), получаем, что $\varepsilon$ должно быть равно нулю.

Доказательство равенства $a_{k}=\eta k(k+1), k \in \mathbb{N}$, аналогично. Мы только наметим схему доказательства. Положим $\eta:=a_{1} / 2$. Из леммы 4.10 и определений (3.17), (3.18) мы получаем рекуррентную формулу

$$
a_{n+1}=2 \eta+((n+1)(n+2)-2) \frac{a_{n}}{n(n+1)}
$$


при $n \geqslant 2$. Выберем $\varepsilon \in \mathbb{R}$ таким образом, чтобы было выполнено равенство $a_{2}=6 \eta+\varepsilon$. Тогда по формуле (4.67) имеем

$$
a_{3}=12 \eta+\frac{10}{6} \varepsilon, \quad a_{4}=20 \eta+\frac{5}{2} \varepsilon, \quad a_{5}=30 \eta+\frac{7}{2} \varepsilon .
$$

С другой стороны, по лемме 4.11

$$
a_{5}=a_{2}+2 a_{3}
$$

Теперь равенство $\varepsilon=0$ следует из (4.68) и (4.69). Как следствие, рекуррентная формула (4.67) выполнена для всех $n \geqslant 1$. Отсюда вытекает требуемое равенство.

В заключение мы рассмотрим случай, когда носитель меры $\nu$ состоит из $l$ точек, где $l \geqslant 2$ конечно. В случае $q=-1$ мы будем дополнительно предполагать, что $l \geqslant 3$. Имеем $a_{1}>0, a_{2}>0, \ldots, a_{l-1}>0, a_{i}=0$ при $i \geqslant l$. Далее, из (3.2) следует, что $c_{1}>0, c_{2}>0, \ldots, c_{l}>0, c_{i}=0$ при $i \geqslant l+1$. Предположим, что выполнено условие $(\mathrm{C})$. Ввиду аналогичной конструкции меры $m_{\nu}^{(n)}$, мы заключаем, что формула (4.67) имеет место при всех $n=1,2, \ldots, l-1$. В частности,

$$
a_{l}=a_{1}+(l(l+1)-2) \frac{a_{l-1}}{(l-1) l} .
$$

Так как $a_{1}>0$ и $a_{l-1}>0$, то отсюда следует неравенство $a_{l}>0$, которое противоречит равенству $a_{l}=0$. Таким образом, условие $(\mathrm{C})$ не может выполняться. Теорема 3.5 доказана.

Доказательство предложения 3.7 мы оставляем читателю.

Теперь теорема 1.2 может быть легко доказана.

Пусть $q=1$. Предположим, что $\mathscr{C} \mathscr{P}=\mathscr{O} \mathscr{C} \mathscr{P}$. Тогда при любых $h \in C_{0}(X)$ и $f^{(n)} \in C_{0}\left(X^{n}\right)$

$$
\langle\omega, h\rangle\left\langle P_{n}(\omega), f^{(n)}\right\rangle \in \mathscr{O} \mathscr{C} \mathscr{P}
$$

(здесь мы воспользовались тем, что произведение многочленов из $\mathscr{C} \mathscr{P}$ снова лежит в $\mathscr{C} \mathscr{P})$. Так как

$$
\mathbf{J}_{2}^{-}(h)\left\langle f^{(n)}, P_{n}(\omega)\right\rangle=\left\langle\mathscr{J}_{2}^{-}(h) f^{(n)}, P_{n-1}(\omega)\right\rangle \in \mathscr{O} \mathscr{C} \mathscr{P},
$$

то из теоремы 3.4 и включения (4.70) следует, что условие (C) выполнено. Это с учетом теоремы 3.5 доказывает (3.22).

Теперь предположим, что выполнено (3.22). Тогда из доказательства теоремы 3.5 имеем $h \in C_{0}(X)$, при этом операторы $\mathscr{J}^{0}(h), \mathscr{J}_{1}^{-}(h)$ отображают $\mathscr{F}_{\text {fin }}\left(C_{0}(X)\right)$ в себя. Как следствие, (4.70) выполнено при всех $f^{(n)} \in C_{0}\left(X^{n}\right)$. Теперь равенство $\mathscr{C} \mathscr{P}=\mathscr{O} \mathscr{C} \mathscr{P}$ может быть доказано аналогично доказательству теоремы 4.1 из [16].

4.5. Доказательство теоремы 3.8. Мы ограничимся доказательством равенства (3.31) (доказательство равенства (3.30) аналогично и проще). Также отметим, что формула (3.32) будет вытекать из (3.26)-(3.31). 
Достаточно показать, что при всех $h \in C_{0}(X)$

$$
\mathbf{J}_{1}^{-}(h) g^{(n)}=\int_{X} d x h(x) \eta \partial_{x}^{\dagger} \partial_{x} \partial_{x} g^{(n)},
$$

где $g^{(n)} \in \mathbf{B}_{0}^{Q}\left(X^{n}\right)$ имеет вид $g^{(n)}=f_{1} \circledast \cdots \circledast f_{n}$ и $f_{1}, \ldots, f_{n} \in B_{0}(X)$. Имеем

$$
g^{(n)}\left(x_{1}, \ldots, x_{n}\right):=\frac{1}{n !} \sum_{\pi \in \mathfrak{S}_{n}} Q_{\pi}\left(x_{1}, \ldots, x_{n}\right) f_{\pi(1)}\left(x_{1}\right) \cdots f_{\pi(n)}\left(x_{n}\right) .
$$

Теперь из (3.29) вытекает, что

$$
\begin{aligned}
\left(\int_{X} d x\right. & \left.h(x) \partial_{x}^{\dagger} \partial_{x} \partial_{x} g^{(n)}\right)\left(x_{1}, \ldots, x_{n-1}\right) \\
= & \operatorname{Sym}_{n-1}\left(\frac{1}{(n-2) !} \sum_{\pi \in \mathfrak{S}_{n}} Q_{\pi}\left(x_{1}, x_{1}, x_{2}, \ldots, x_{n-1}\right)\right. \\
& \left.\times\left(h f_{\pi(1)} f_{\pi(2)}\right)\left(x_{1}\right) f_{\pi(3)}\left(x_{2}\right) \cdots f_{\pi(n)}\left(x_{n-1}\right)\right) \\
= & \sum_{1 \leqslant i<j \leqslant n} \frac{1}{(n-2) !} \sum_{\substack{\pi \in \mathfrak{S}_{n} \\
\pi\{1,2\}=\{i, j\}}} \operatorname{Sym}_{n-1}\left(Q_{\pi}\left(x_{1}, x_{1}, x_{2}, \ldots, x_{n-1}\right)\right. \\
& \left.\times\left(h f_{i} f_{j}\right)\left(x_{1}\right) f_{\pi(3)}\left(x_{2}\right) \cdots f_{\pi(n)}\left(x_{n-1}\right)\right) .
\end{aligned}
$$

В силу (3.7) для любой перестановки $\pi \in \mathfrak{S}_{n}$ такой, что $\pi\{1,2\}=\{i, j\}$ при $i<j$, и любого $\left(x_{1}, x_{2}, \ldots, x_{n-1}\right) \in X^{n-1}$ имеем

$$
Q_{\pi}\left(x_{1}, x_{1}, x_{2}, \ldots, x_{n-1}\right)=Q_{\sigma_{i j}(\pi)}\left(x_{1}, x_{2}, \ldots, x_{n-1}\right) .
$$

Здесь перестановка $\sigma_{i j}(\pi) \in \mathfrak{S}_{n-1}$ определяется следующим образом:

$$
\sigma_{i j}(\pi)(1):=j
$$

и для $k=2, \ldots, n-1$

$$
\sigma_{i j}(\pi)(k):= \begin{cases}\pi(k+1), & \text { если } \pi(k+1)<i, \\ \pi(k+1)-1, & \text { если } \pi(k+1)>i\end{cases}
$$

Ввиду (4.72), для любого $\pi \in \mathfrak{S}_{n}$ такого, что $\pi\{1,2\}=\{i, j\}$ при $i<j$, мы имеем

$$
\begin{aligned}
& Q_{\pi}\left(x_{1}, x_{1}, x_{2}, \ldots, x_{n-1}\right)\left(h f_{i} f_{j}\right)\left(x_{1}\right) f_{\pi(3)}\left(x_{2}\right) \cdots f_{\pi(n)}\left(x_{n-1}\right) \\
& =Q_{\sigma_{i j}(\pi)}\left(x_{1}, x_{2}, \ldots, x_{n-1}\right)\left(f_{1} \otimes \cdots \otimes f_{i-1} \otimes f_{i+1} \otimes \cdots \otimes f_{j-1}\right. \\
& \left.\otimes\left(h f_{i} f_{j}\right) \otimes f_{j+1} \otimes \cdots \otimes f_{n}\right)\left(x_{\sigma_{i j}(\pi)^{-1}(1)}, \ldots, x_{\sigma_{i j}(\pi)^{-1}(n-1)}\right) \\
& =\Psi_{\sigma_{i j}(\pi)}\left(f_{1} \otimes \cdots \otimes f_{i-1} \otimes f_{i+1} \otimes \cdots \otimes f_{j-1}\right. \\
& \left.\otimes\left(h f_{i} f_{j}\right) \otimes f_{j+1} \otimes \cdots \otimes f_{n}\right)\left(x_{1}, \ldots, x_{n-1}\right) .
\end{aligned}
$$


Отсюда, используя (4.5) и (4.10), получаем, что

$$
\begin{gathered}
\operatorname{Sym}\left(Q_{\pi}\left(x_{1}, x_{1}, x_{2}, \ldots, x_{n-1}\right)\left(h f_{i} f_{j}\right)\left(x_{1}\right) f_{\pi(3)}\left(x_{2}\right) \cdots f_{\pi(n)}\left(x_{n-1}\right)\right) \\
=\left(f_{1} \circledast \cdots \circledast f_{i-1} \circledast f_{i+1} \circledast \cdots \circledast f_{j-1}\right. \\
\left.\circledast\left(h f_{i} f_{j}\right) \circledast f_{j+1} \circledast \cdots \circledast f_{n}\right)\left(x_{1}, \ldots, x_{n-1}\right) .
\end{gathered}
$$

Окончательно, применяя (4.71) и (4.73), находим, что

$$
\begin{aligned}
\int_{X} d x & h(x) \partial_{x}^{\dagger} \partial_{x} \partial_{x} g^{(n)} \\
\quad=2 & \sum_{1 \leqslant i<j \leqslant n} f_{1} \circledast \cdots \circledast f_{i-1} \circledast f_{i+1} \circledast \cdots \circledast f_{j-1} \circledast\left(h f_{i} f_{j}\right) \circledast f_{j+1} \circledast \cdots \circledast f_{n},
\end{aligned}
$$

откуда вытекает равенство (3.31).

\section{Список литературы}

[1] L. Accardi, U. Franz, M. Skeide, "Renormalized squares of white noise and other non-Gaussian noises as Lévy processes on real Lie algebras", Comm. Math. Phys., 228:1 (2002), 123-150.

[2] S. Albeverio, Yu. G. Kondratiev, M. Röckner, "Analysis and geometry on configuration spaces", J. Funct. Anal., 154:2 (1998), 444-500.

[3] M. Anshelevich, "q-Lévy processes", J. Reine Angew. Math., 2004:576 (2004), 181-207.

[4] M. Anshelevich, "Free Meixner states", Comm. Math. Phys., 276:3 (2007), 863-899.

[5] M. Anshelevich, "Orthogonal polynomials with a resolvent-type generating function", Trans. Amer. Math. Soc., 360:8 (2008), 4125-4143.

[6] S. T. Belinschi, M. Bożejko, F. Lehner, R. Speicher, "The normal distribution is 円-infinitely divisible", Adv. Math., 226:4 (2011), 3677-3698.

[7] Y.M. Berezansky, "Commutative Jacobi fields in Fock space", Integral Equations Operator Theory, 30:2 (1998), 163-190.

[8] Ю. М. Березанский, Ю. Г. Кондратьев, Спектральные методы в бесконечномерном анализе, Наукова думка, Киев, 1988, 680 с.; англ. пер.: Y. M. Berezansky, Y. G. Kondratiev, Spectral methods in infinite-dimensional analysis, v. 1, 2, Math. Phys. Appl. Math., 12, Kluwer Acad. Publ., Dordrecht, 1995, xviii+576 pp., viii+432 pp.

[9] Y. M. Berezansky, E. Lytvynov, D. A. Mierzejewski, "The Jacobi field of a Lévy process", Укр. матем. журн., 55:5 (2003), 706-710; Ukrainian Math. J., 55:5 (2003), 853-858.

[10] P. Biane, "Processes with free increments", Math. Z., 227:1 (1998), 143-174.

[11] M. Bożejko, "Deformed Fock spaces, Hecke operators and monotone Fock space of Muraki", Demonstratio Math., 45:2 (2012), 399-413.

[12] M. Bożejko, W. Bryc, "On a class of free Lévy laws related to a regression problem", J. Funct. Anal., 236:1 (2006), 59-77.

[13] M. Bożejko, N. Demni, "Generating functions of Cauchy-Stieltjes type for orthogonal polynomials", Infin. Dimens. Anal. Quantum Probab. Relat. Top., 12:1 (2009), 91-98.

[14] M. Bożejko, T. Hasebe, "On free infinite divisibility for classical Meixner distributions", Probab. Math. Statist., 33:2 (2013), 363-375. 
[15] M. Bożejko, B. Kümmerer, R. Speicher, " $q$-Gaussian processes: non-commutative and classical aspects", Comm. Math. Phys., 185:1 (1997), 129-154.

[16] M. Bożejko, E. Lytvynov, "Meixner class of non-commutative generalized stochastic processes with freely independent values. I. A characterization", Comm. Math. Phys., 292:1 (2009), 99-129.

[17] M. Bożejko, E. Lytvynov, "Meixner class of non-commutative generalized stochastic processes with freely independent values. II. The generating function", Comm. Math. Phys., 302:2 (2011), 425-451.

[18] M. Bożejko, E. Lytvynov, J. Wysoczański, "Noncommutative Lévy processes for generalized (particularly anyon) statistics", Comm. Math. Phys., 313:2 (2012), 535-569.

[19] M. Bożejko, R. Speicher, "An example of a generalized Brownian motion", Comm. Math. Phys., 137:3 (1991), 519-531.

[20] E. Brüning, "When is a field a Jacobi-field? A characterization of states on tensor algebras", Publ. Res. Inst. Math. Sci., 22:2 (1986), 209-246.

[21] E. Brüning, "On the construction of fields and the topological role of Jacobi fields", Rep. Math. Phys., 21:2 (1985), 143-158.

[22] W. Bryc, J. Wesołowski, "Conditional moments of $q$-Meixner processes", Probab. Theory Related Fields, 131:3 (2005), 415-441.

[23] T.S. Chihara, An introduction to orthogonal polynomials, Math. Appl., 13, Gordon and Breach Science Publishers, New York-London-Paris, 1978, xii+249 pp.

[24] S. Das, Orthogonal decompositions for generalized stochastic processes with independent values, PhD thesis, Swansea Univ., Swansea, UK, 2012.

[25] G. Di Nunno, B. Øksendal, F. Proske, Malliavin calculus for Lévy processes with applications to finance, Universitext, Springer-Verlag, Berlin, 2009, xiv+413 pp.

[26] I. M. Gel'fand, M. I. Graev, A. M. Vershik, "Models of representations of current groups", Representations of Lie groups and Lie algebras (Budapest, 1971), Akad. Kiadó, Budapest, 1985, 121-179.

[27] И. М. Гельфанд, Н.Я. Виленкин, Обобщенные функиии. Вып. 4: Некоторые применения гармонического анализа. Оснащенные гильбертовы пространства, Физматгиз, М., 1961, 472 с.; англ. пер.: I. M. Gel'fand, N. Ya. Vilenkin, Generalized functions, v. 4: Applications of harmonic analysis, Academic Press, New York-London, 1964, xiv+384 pp.

[28] G. A. Goldin, S. Majid, "On the Fock space for nonrelativistic anyon fields and braided tensor products", J. Math. Phys., 45:10 (2004), 3770-3787.

[29] G. A. Goldin, D. H. Sharp, "Diffeomorphism groups, anyon fields, and $q$ commutators", Phys. Rev. Lett., 76:8 (1996), 1183-1187.

[30] B. Grigelionis, "Processes of Meixner type", Lithuanian Math. J., 39:1 (1999), 33-41.

[31] D. Hagedorn, Y. Kondratiev, E. Lytvynov, A. Vershik, Laplace operators in gamma analysis, 2014, 27 pp., arXiv: 1411.0162.

[32] D. Hagedorn, Y. Kondratiev, T. Pasurek, M. Röckner, "Gibbs states over the cone of discrete measures", J. Funct. Anal., 264:11 (2013), 2550-2583.

[33] T. Hida, H.-H. Kuo, J. Potthoff, L. Streit, White noise. An infinite-dimensional calculus, Math. Appl., 253, Kluwer Acad. Publ., Dordrecht, 1993, xiv+516 pp.

[34] K. Itô, "Spectral type of the shift transformation of differential processes with stationary increments", Trans. Amer. Math. Soc., 81:2 (1956), 253-263.

[35] Y. Ito, I. Kubo, "Calculus on Gaussian and Poisson white noises", Nagoya Math. J., 111 (1988), 41-84.

[36] O. Kallenberg, Random measures, 3rd ed., Akademie-Verlag, Berlin; Academic Press, Inc., London, 1983, 187 pp. 
[37] Y. G. Kondratiev, J. L. da Silva, L. Streit, G. F. Us, "Analysis on Poisson and gamma spaces", Infin. Dimens. Anal. Quantum Probab. Relat. Top., 1:1 (1998), 91-117.

[38] Y. G. Kondratiev, E. W. Lytvynov, "Operators of gamma white noise calculus", Infin. Dimens. Anal. Quantum Probab. Relat. Top., 3:3 (2000), 303-335.

[39] A. Liguori, M. Mintchev, "Fock representations of quantum fields with generalized statistics", Comm. Math. Phys., 169:3 (1995), 635-652.

[40] E. W. Lytvynov, "Multiple Wiener integrals and non-Gaussian white noises: a Jacobi field approach", Methods Funct. Anal. Topology, 1:1 (1995), 61-85.

[41] E. Lytvynov, "Polynomials of Meixner's type in infinite dimensions - Jacobi fields and orthogonality measures", J. Funct. Anal., 200:1 (2003), 118-149.

[42] E. Lytvynov, "Orthogonal decompositions for Lévy processes with an application to the gamma, Pascal, and Meixner processes", Infin. Dimens. Anal. Quantum Probab. Relat. Top., 6:1 (2003), 73-102.

[43] E. Lytvynov, I. Rodionova, "Lowering and raising operators for the free Meixner class of orthogonal polynomials", Infin. Dimens. Anal. Quantum Probab. Relat. Top., 12:3 (2009), 387-399.

[44] J. Meixner, "Orthogonale Polynomsysteme mit einem besonderen Gestalt der erzeugenden Funktion", J. London Math. Soc., s1-9:1 (1934), 6-13.

[45] D. Nualart, W. Schoutens, "Chaotic and predictable representations for Lévy processes", Stochastic Process. Appl., 90:1 (2000), 109-122.

[46] М. Рид, Б. Саймон, Методъ современной математической физики, т. 2: Гармонический анализ. Самосопряженность, Мир, М., 1978, 395 с.; пер. с англ.: M. Reed, B. Simon, Methods of modern mathematical physics, v. II: Fourier analysis, self-adjointness, Academic Press, New York-London, 1975, xv+361 pp.

[47] I. Rodionova, "Analysis connected with generating functions of exponential type in one and infinite dimensions", Methods Funct. Anal. Topology, 11:3 (2005), 275-297.

[48] W. Schoutens, Stochastic processes and orthogonal polynomials, Lecture Notes in Statist., 146, Springer-Verlag, New York, 2000, xiv+163 pp.

[49] W. Schoutens, J. L. Teugels, "Lévy processes, polynomials and martingales", Comm. Statist. Stochastic Models, 14:1-2 (1998), 335-349.

[50] А. В. Скороход, Интегрирование в гильбертовом пространстве, Наука, М., 1975, 231 с.; англ. пер.: A. V. Skorohod, Integration in Hilbert space, Ergeb. Math. Grenzgeb., 79, Springer-Verlag, New York-Heidelberg, 1974, xii+177 pp.

[51] D. Surgailis, "On multiple Poisson stochastic integrals and associated Markov semigroups", Probab. Math. Statist., 3:2 (1984), 217-239.

[52] N. Tsilevich, A. Vershik, M. Yor, "An infinite-dimensional analogue of the Lebesgue measure and distinguished properties of the gamma process", J. Funct. Anal., 185:1 (2001), 274-296.

[53] А. М. Вершик, “Существует ли мера Лебега в бесконечномерном пространстве?", Анализ и особенности. Часть 2, Сборник статей. К 70-летию со дня рождения академика Владимира Игоревича Арнольда, Тр. МИАН, 259, Наука, М., 2007, 256-281; англ. пер.: A. M. Vershik, "Does there exist a Lebesgue measure in the infinite-dimensional space?", Proc. Steklov Inst. Math., 259 (2007), 248-272.

[54] А. М. Вершик, И. М. Гельфанд, М. И. Граев, "Представления группы $S L(2, \mathbf{R})$, где $\mathbf{R}$ - кольцо функций", УМH, 28:5(173) (1973), 83-128; англ. пер.: А. M. Vershik, I. M. Gel'fand, M. I. Graev, "Representations of the group $S L(2, \mathbf{R})$, where $\mathbf{R}$ is a ring of functions", Russian Math. Surveys, 28:5 (1973), 87-132; Representation theory, London Math. Soc. Lecture Note Ser., 69, Cambridge Univ. Press, Cambridge-New York, 1982, 15-60. 
[55] А. М. Вершик, И. М. Гельфанд, М. И. Граев, "Представления группы диффеоморфизмов", УМH, 30:6(186) (1975), 3-50; англ. пер.: А. M. Vershik, I. M. Gel'fand, M. I. Graev, "Representations of the group of diffeomorphisms", Russian Math. Surveys, 30:6 (1975), 1-50.

[56] А. М. Вершик, И. М. Гельфанд, М. И. Граев, "Коммутативная модель представления группы токов $S L(2, \mathbf{R})^{X}$, связанная с унипотентной подгруппой”, Функи. анализ и его прил., 17:2 (1983), 70-72; англ. пер.: A. M. Vershik, I. M. Gel'fand, M. I. Graev, "A commutative model of representation of the group of flows $S L(2, \mathbf{R})^{X}$ that is connected with a unipotent subgroup", Funct. Anal. Appl., 17:2 (1983), 137-139.

[57] А. М. Вершик, Н.В. Цилевич, "Фоковские факторизации и разложения пространств $L^{2}$ над общими процессами Леви”, УМH, 58:3(351) (2003), 3-50; англ. пер.: A. M. Vershik, N. V. Tsilevich, "Fock factorizations, and decompositions of the $L^{2}$ spaces over general Lévy processes", Russian Math. Surveys, 58:3 (2003), 427-472.

\section{Марек Божейко}

Поступила в редакцию

(Marek Bożejko)

01.12 .2014

Institute of Mathematics, Wrocław University,

Wrocław, Poland

E-mail: bozejko@math.uni.wroc.pl

\section{Евгений Вильямович Литвинов}

(Eugene W. Lytvynov)

Swansea University, Swansea, UK

E-mail: e.lytvynov@swansea.ac.uk

\section{Ирина Викторовна Родионова}

(Irina V. Rodionova)

Swansea University, Swansea, UK

E-mail: i.rodionova@swansea.ac.uk 